\title{
Nucleophilic Substitution Reactions of Sulfur-Substituted Cyclohexanone Acetals: An Analysis of the Factors Controlling Selectivity
}

\author{
Susan B. Billings and K. A. Woerpel* \\ Department of Chemistry, University of California \\ Irvine, CA 92697-2025
}

Table of Contents:

\section{Supporting Information}

I. General Experimental

II. Synthesis of Sulfur Substituted Cyclohexanone Acetals

A. Unconstrained Model: 2-Thiophenyl Acetal

B. Constrained Model: tert-Butyl Acetal

C. Conformationally-Biased Model: 4,4-Dimethyl Acetal S-5

D. Sterically Encumbering Model: 5,5-Dimethyl Acetal $\quad S-6$

III. Synthesis of 2-Heteroatom Substituted Cyclohexanone Acetals

A. Fluoro Acetal

B. Chloro Acetal

C. Iodo Acetal

D. Phenoxy Acetal

$S-9$

IV. Nucleophilic Substitution Reactions

$S-10$

V. Stereochemical Proofs of Nucleophilic Substitution products

$S-18$

VI. Bibliography

$S-25$

VII. Analytical Data

$S-26$

\section{General Experimental}

${ }^{1} \mathrm{H}$ NMR and ${ }^{13} \mathrm{C}$ NMR spectra were recorded at ambient temperature at $500 \mathrm{MHz}$ and $125 \mathrm{MHz}$, respectively. The data are reported as follows: chemical shift in ppm from internal tetramethylsilane on the $\square$ scale, multiplicity ( $\mathrm{br}=$ broad, $\mathrm{s}=$ singlet, $\mathrm{d}=$ doublet, $\mathrm{t}=$ triplet, $\mathrm{q}=$ quartet, $\mathrm{m}=$ multiplet), coupling constants $(\mathrm{Hz})$ and integration. High resolution mass spectra were obtained by peak matching. Melting points were reported uncorrected. All reactions were carried out under an atmosphere of nitrogen or argon in glassware, which had been flamed dried under a stream of gas. Unless otherwise noted, all reagents were commercially available obtained and, where appropriate, purified prior to use. THF, $\mathrm{Et}_{2} \mathrm{O}$, and $\mathrm{CH}_{2} \mathrm{Cl}_{2}$ were dried by filtration through alumina. ${ }^{1}$

\section{Synthesis of Sulfur Substituted Cyclohexanone Acetals}

\section{A. Unconstrained Model:}




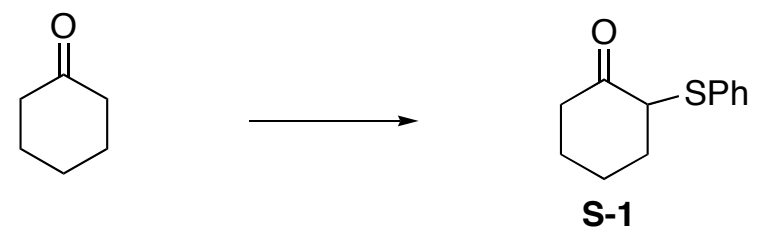

General Procedure for Lithium Diisopropylamide (LDA) Preparation: To a cooled $\left(-78{ }^{\circ} \mathrm{C}\right)$ solution of diisopropylamine $(1.00$ equiv) in THF $(0.50 \mathrm{M})$ was added $n$-BuLi (1.00 equiv). The reaction mixture was warmed to $0{ }^{\circ} \mathrm{C}$ with an ice water bath and maintained at this temperature for $45 \mathrm{~min}$.

General Procedure for Phenylsulfenylation of Cyclohexanones: ${ }^{2,3}$ A cooled $\left(-78{ }^{\circ} \mathrm{C}\right)$ solution of lithium diisopropylamide in THF $(0.50 \mathrm{M})$ was treated with a solution of cyclohexanone (1.00 equiv) in THF $(0.80 \mathrm{M})$. The solution was maintained at $-78{ }^{\circ} \mathrm{C}$ for $1 \mathrm{~h}$. The cold reaction mixture (enolate solution) was added by cannula to a solution of diphenyl disulfide (1.1 equiv) in THF $(0.70 \mathrm{M})$ at ambient temperature. After $4 \mathrm{~h}$ at $22^{\circ} \mathrm{C}$, the reaction mixture was diluted with EtOAc and poured into a separatory funnel with $15 \% \mathrm{HCl}(1$ $\mathrm{mL}$ per mmol of diphenyl disulfide). The organic layer was washed with a saturated sodium bicarbonate solution ( $1 \mathrm{~mL}$ per mmol of diphenyl disulfide), dried $\left(\mathrm{MgSO}_{4}\right)$, and concentrated in vacuo to provide a bright yellow residue.

2-Phenylsulfanylcyclohexanone S-1. ${ }^{3}$ The standard phenylsulfenylation procedure was followed with cyclohexanone $(5.00 \mathrm{~g}, 51.0 \mathrm{mmol})$ in $64 \mathrm{~mL}$ of THF with LDA $(61.0 \mathrm{mmol})$, diphenyl disulfide $(12.20 \mathrm{~g}, 56.0$ mmol). Purification of the resultant residue by silica gel chromatography (0:100 to 5:95 EtOAc:hexanes) yielded product $\mathbf{S - 1}$ as a yellow oil $(5.52 \mathrm{~g}, 50 \%)$. The spectral data correlates with the previously reported data for $\mathbf{S - 1}$. $^{3}$<smiles>O=C1CCCCC1Sc1ccccc1</smiles>

S-1<smiles>COC1(OC)CCCCC1Sc1ccccc1</smiles>

7

General Procedure for Acetalization of Cyclohexanones: ${ }^{4}$ A solution of cyclohexanone in $\mathrm{MeOH}$ $(0.15 \mathrm{M})$ was treated with trimethyl orthoformate (4.00 equiv) and 3 drops of concentrated $\mathrm{H}_{2} \mathrm{SO}_{4}$. The reaction mixture was heated to $50{ }^{\circ} \mathrm{C}$ and stirred for $12 \mathrm{~h}$ before it was poured into a separatory funnel containing saturated aqueous $\mathrm{NaHCO}_{3}(1 \mathrm{~mL}$ per mmol of cyclohexanone). The aqueous layer was extracted with 3 portions of $\mathrm{CH}_{2} \mathrm{Cl}_{2}$ (1 mmol per cyclohexanone). The combined organic layers were washed with a saturated sodium chloride solution, dried $\left(\mathrm{Na}_{2} \mathrm{SO}_{4}\right)$, and concentrated in vacuo to provide a pale yellow residue.

Acetal 7. ${ }^{5}$ The standard acetalization procedure was followed with 2-phenylsulfanylcyclohexanone S-1 (4.34 g, $21.0 \mathrm{mmol})$ in $64 \mathrm{~mL}$ of $\mathrm{MeOH}$ with trimethylorthoformate $(11.5 \mathrm{~mL}, 105.3 \mathrm{mmol})$ and $\mathrm{H}_{2} \mathrm{SO}_{4}(3$ drops). Purification of the resultant residue by silica gel chromatography (3:98 EtOAc:hexanes) yielded product 7 as a yellow oil $(5.21 \mathrm{~g}, 98 \%)$. The spectral data correlates with the previously reported data for 7: ${ }^{1} \mathrm{H}$ NMR (500 MHz, $\left.\mathrm{CDCl}_{3}\right) \square 7.05-7.46(\mathrm{~m}, 5 \mathrm{H}), 3.63(\mathrm{~m}, 1 \mathrm{H}), 3.25(\mathrm{~s}, 3 \mathrm{H}), 3.21(\mathrm{~s}, 3 \mathrm{H}), 1.81(\mathrm{~m}, 3 \mathrm{H}), 1.64$ $(\mathrm{m}, 3 \mathrm{H}), 1.38(\mathrm{~m}, 2 \mathrm{H}) ;{ }^{13} \mathrm{C} \mathrm{NMR}\left(125 \mathrm{MHz}, \mathrm{CDCl}_{3}\right) \square 135.8,131.9,129.2,126.9,101.2,50.7,48.1,47.6,28.3$, 27.9, 22.6, 20.2; HRMS (EI) $\mathrm{m} / z$ calcd for $\mathrm{C}_{14} \mathrm{H}_{20} \mathrm{O}_{2} \mathrm{~S}[\mathrm{M}]^{+} 252.1184$, found 252.1183.

\section{B. Constrained Model:}




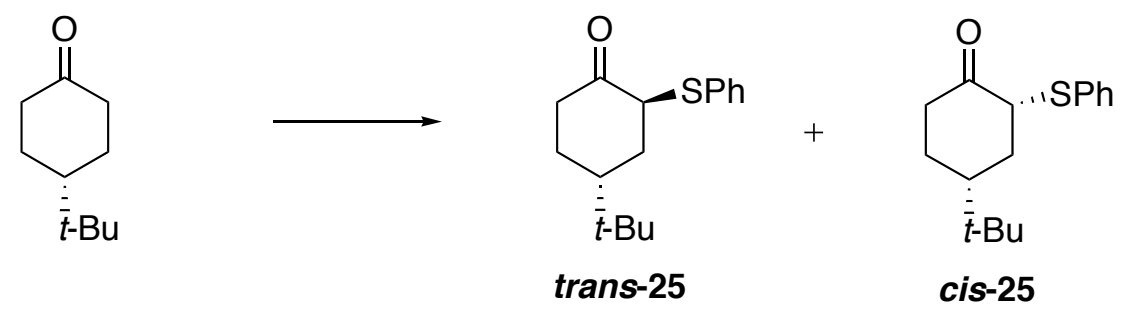

4-tert-Butyl-2-phenylsulfanylcyclohexanones trans-25 and cis-25. ${ }^{3,6}$ The standard phenylsulfenylation procedure was followed with $t$-butylcyclohexanone $(15.0 \mathrm{~g}, 97.2 \mathrm{mmol})$ in $120 \mathrm{~mL}$ of THF with LDA (120.0 mmol), diphenyl disulfide (23.35 g, $110.0 \mathrm{mmol})$. GC and ${ }^{1} \mathrm{H}$ NMR spectroscopic analysis indicated a pair of diastereomers in a 3:1 ratio. Purification of the resultant residue by silica gel chromatography (0:100 to 5:95 EtOAc:hexanes) yielded a mixture of products trans-25 and cis-25 as a yellow oil (18.23 g, 60\%). The spectral data correlates with the previously reported data for $25 .{ }^{3}$ Separation of the diastereomers was achieved by reduction with lithium aluminum hydride and subsequent oxidation.<smiles>CC(C)(C)[C@H]1CCC(=O)C([SbH2])C1</smiles>

25<smiles>CC(C)(C)[Mg]c1ccccc1</smiles>

26<smiles>CC(C)(C)[C@H]1CC[C@@H](O)[C@H](S)C1</smiles>

$27 a$<smiles>CC(C)(C)[C@H]1CC[C@@H](O)[C@H]([SnH2])C1</smiles>

$27 b$

4-tert-Butyl-2-phenylsulfanylcyclohexanols $26,27 \mathbf{a}$ and $27 \mathbf{b}$. To a cooled $\left(0{ }^{\circ} \mathrm{C}\right)$ solution of a diastereomeric mixture of ketones 25 (4.00 g, $15.3 \mathrm{mmol})$ in $192 \mathrm{~mL}$ of THF was added $\mathrm{LiAlH}_{4}(2.90 \mathrm{~g}, 77.0$ mmol). The gray mixture was stirred at $22{ }^{\circ} \mathrm{C}$ for $12 \mathrm{~h}$. The reaction mixture was cooled to $0{ }^{\circ} \mathrm{C}$ and treated with $\mathrm{H}_{2} \mathrm{O}(75 \mathrm{~mL}), 5 \% \mathrm{NaOH}$ solution $(75 \mathrm{~mL})$, and $\mathrm{H}_{2} \mathrm{O}(3 \times 75 \mathrm{~mL})$. A small portion of $\mathrm{Na}_{2} \mathrm{SO}_{4}(200 \mathrm{mg})$ was added and the reaction mixture was stirred at $22{ }^{\circ} \mathrm{C}$ for $20 \mathrm{~min}$. The reaction mixture was filtered and concentrated in vacuo to provide a white residue as a mixture of diastereomers in a 13:5:2 ratio. Separation of the isomers was achieved by purification of the resultant residue by silica gel chromatography (0:100 to 5:95 EtOAc:hexanes) to yield 26, 27a and $\mathbf{2 7 b}$ as a white solid (2.01 g, 50\% overall yield). The spectral data correlates with the previously reported data for alcohols $\mathbf{2 6}, \mathbf{2 7} \mathbf{a}$, and $\mathbf{2 7} \mathbf{b}$. ${ }^{6}$

Major Isomer (26). ${ }^{1} \mathrm{H} \mathrm{NMR}\left(500 \mathrm{MHz}, \mathrm{CDCl}_{3}\right) \square 7.20-7.49(\mathrm{~m}, 5 \mathrm{H}), 3.75$ (ddd, $\left.J=4.4,11.7,4.4 \mathrm{~Hz}, 1 \mathrm{H}\right)$, $3.68(\mathrm{~m}, 1 \mathrm{H}), 2.26(\mathrm{~d}, J=10.3 \mathrm{~Hz}, 1 \mathrm{H}), 2.13(\mathrm{~m}, 1 \mathrm{H}), 1.88(\mathrm{~m}, 1 \mathrm{H}), 1.78(\mathrm{~m}, 1 \mathrm{H}), 1.45(\mathrm{~m}, 3 \mathrm{H}), 1.05(\mathrm{~m}, 1 \mathrm{H})$, 0.86 (s, 9H); ${ }^{13} \mathrm{C}$ NMR $\left(125 \mathrm{MHz}, \mathrm{CDCl}_{3}\right) \square 136.8,131.9,129.3,127.2,71.9,57.8,41.8,33.2,32.5,32.3,27.9$, 25.7; HRMS (CI) $m / z$ calcd for $\mathrm{C}_{16} \mathrm{H}_{24} \mathrm{KOS}[\mathrm{M}+\mathrm{K}]^{+} 287.1446$, found 287.1451.

Mass spectrometry data were obtained for Minor Isomer 27a and Minor Isomer 27b as a mixture of diastereomers: HRMS (CI) $m / z$ calcd for $\mathrm{C}_{16} \mathrm{H}_{24} \mathrm{NaOS}[\mathrm{M}+\mathrm{Na}]^{+} 287.1446$, found 287.1453.

Minor Isomer (27a). ${ }^{1} \mathrm{H}$ NMR $\left(500 \mathrm{MHz}, \mathrm{CDCl}_{3}\right) \square 7.29-7.49(\mathrm{~m}, 5 \mathrm{H}), 3.28(\mathrm{td}, J=10.7,4.5 \mathrm{~Hz}, 1 \mathrm{H}), 2.93(\mathrm{~d}$, $J=1.5 \mathrm{~Hz}, 1 \mathrm{H}), 2.81(\mathrm{~m}, 1 \mathrm{H}), 2.15(\mathrm{~m}, 2 \mathrm{H}), 1.77(\mathrm{~m}, 1 \mathrm{H}), 1.33(\mathrm{ddt}, J=3.7,10.9,13.0 \mathrm{~Hz}, 1 \mathrm{H}), 1.13(\mathrm{~m}, 2 \mathrm{H})$, $1.01(\mathrm{~m}, 1 \mathrm{H}), 0.82(\mathrm{~s}, 9 \mathrm{H}) ;{ }^{13} \mathrm{C}$ NMR $\left(125 \mathrm{MHz}, \mathrm{CDCl}_{3}\right) \square 133.6,133.2,129.2,127.9,77.8,57.3,48.5,34.4$, $33.9,32.7,27.8,25.5$.

Minor Isomer (27b). ${ }^{1} \mathrm{H}$ NMR (500 MHz, $\left.\mathrm{CDCl}_{3}\right) \square 7.24-7.50(\mathrm{~m}, 5 \mathrm{H}), 3.71(\mathrm{~m}, 1 \mathrm{H}), 3.23$ (ddd, $J=2.3,3.8$, $12.9 \mathrm{~Hz}, 1 \mathrm{H}), 2.48(\mathrm{~s}, 1 \mathrm{H}), 2.05(\mathrm{~m}, 1 \mathrm{H}), 1.87(\mathrm{~m}, 1 \mathrm{H}), 1.47(\mathrm{~m}, 4 \mathrm{H}), 1.13(\mathrm{~m}, 1 \mathrm{H}), 0.88(\mathrm{~s}, 9 \mathrm{H})$. 
<smiles>CC(C)(C)[C@H]1CC[C@@H](O)[C@H](S)C1</smiles>

26<smiles>CC(C)(C)[C@H]1CCC(=O)[C@H](S)C1</smiles>

trans-25

trans-4-tert-Butyl-2-phenylsulfanylcyclohexanone $25 ., 6$ To a solution of Dess-Martin periodinane ${ }^{7,8}$ $(0.642 \mathrm{~g}, 1.50 \mathrm{mmol})$ in $6.0 \mathrm{~mL}$ of $\mathrm{CH}_{2} \mathrm{Cl}_{2}$ was added a solution of alcohol $26(0.363 \mathrm{~g}, 1.40 \mathrm{mmol})$ in $6 \mathrm{~mL}$ of $\mathrm{CH}_{2} \mathrm{Cl}_{2}$. After stirring at $22{ }^{\circ} \mathrm{C}$ for $15 \mathrm{~h}$, the reaction mixture was diluted with $12 \mathrm{~mL}$ of $\mathrm{Et}_{2} \mathrm{O}$ and then poured into a separatory funnel containing $15 \mathrm{~mL}$ of a $1: 1 \mathrm{NaHCO}_{3}: \mathrm{Na}_{2} \mathrm{SO}_{4}$ solution. The organic layer was washed with $\mathrm{NaHCO}_{3}$, three portions of $\mathrm{H}_{2} \mathrm{O}(15 \mathrm{~mL})$, dried $\left(\mathrm{Na}_{2} \mathrm{SO}_{4}\right)$, and concentrated in vacuo. ${ }^{1} \mathrm{H} \mathrm{NMR}$ spectroscopic analysis of the unpurified product indicated $95 \%$ purity of the yellow oil $(0.614 \mathrm{~g}, 96 \%)$. The spectral data correlates with the previously reported data for trans-25: ${ }^{3,6}{ }^{1} \mathrm{H} \mathrm{NMR}\left(500 \mathrm{MHz}, \mathrm{CDCl}_{3}\right) \square 7.48(\mathrm{~m}$, 2H), $7.02(\mathrm{~m}, 2 \mathrm{H}), 6.92(\mathrm{~m}, 1 \mathrm{H}), 3.73(\mathrm{dt}, J=4.6,2.2 \mathrm{~Hz}, 1 \mathrm{H}), 2.90(\mathrm{td}, J=14.2,6.1 \mathrm{~Hz}, 1 \mathrm{H}), 2.13$ (ddt, $J=$ $14.3,4.1,2.1 \mathrm{~Hz}, 1 \mathrm{H}), 2.00(\mathrm{dq}, J=14.0,3.0 \mathrm{~Hz}, 1 \mathrm{H}), 1.69(\mathrm{tt}, J=12.0,3.0 \mathrm{~Hz}, 1 \mathrm{H}), 1.58(\mathrm{~m}, 1 \mathrm{H}), 1.44(\mathrm{td}, J=$ 13.0, $4.8 \mathrm{~Hz}, 1 \mathrm{H}), 0.99$ (ddt, $J=13.0,12.7,4.2 \mathrm{~Hz}, 1 \mathrm{H}), 0.67(\mathrm{~s}, 9 \mathrm{H}) ;{ }^{13} \mathrm{C} \mathrm{NMR}\left(125 \mathrm{MHz}, \mathrm{CDCl}_{3}\right) \square 209.0$, 134.3, 131.8, 129.4, 127.9, 55.0, 41.9, 36.9, 34.2, 32.5, 28.3, 27.8; HRMS (CI) $m / z$ calcd for $\mathrm{C}_{16} \mathrm{H}_{23} \mathrm{OS}[\mathrm{M}+\mathrm{H}]^{+}$ 263.1470, found 263.1471 .<smiles>CC(C)(C)[C@H]1CCC(=O)[C@H](S)C1</smiles>

trans-25<smiles>COC1(OC)CCC(C(C)(C)C)CC1[Sc+]c1ccccc1</smiles>

19

tert-Butyl acetal 19. The standard acetalization procedure was followed with trans-25 (2.50 g, 9.60 $\mathrm{mmol}$ ) in $32 \mathrm{~mL}$ of $\mathrm{MeOH}$ with trimethyl orthoformate $(11.0 \mathrm{~mL}, 96.0 \mathrm{mmol})$ and $\mathrm{H}_{2} \mathrm{SO}_{4}$ (3 drops). Purification of the resultant residue by silica gel chromatography (0:100 to 5:95 EtOAc:hexanes) yielded product 19 as a yellow oil (2.25 g, 77\%): ${ }^{1} \mathrm{H}$ NMR (500 MHz, $\left.\mathrm{CDCl}_{3}\right) \square 7.21-7.46(\mathrm{~m}, 5 \mathrm{H}), 3.63(\mathrm{~m}, 1 \mathrm{H}), 3.25$ $(\mathrm{s}, 3 \mathrm{H}), 3.21(\mathrm{~s}, 3 \mathrm{H}), 1.91(\mathrm{~m}, 1 \mathrm{H}), 1.68(\mathrm{~m}, 4 \mathrm{H}), 1.53(\mathrm{~m}, 1 \mathrm{H}), 1.14(\mathrm{~m}, 1 \mathrm{H}), 0.77(\mathrm{~s}, 9 \mathrm{H}) ;{ }^{13} \mathrm{C} \mathrm{NMR}(125 \mathrm{MHz}$, $\left.\mathrm{CDCl}_{3}\right) \square 135.4,132.9,129.2,127.3,101.2,51.2,48.0,47.8,40.8,32.2,28.9,28.6,27.8,23.5$; IR (thin film) 2960, 1478, 1208, $1023 \mathrm{~cm}^{-1}$; HRMS (CI) $\mathrm{m} / z$ calcd for $\mathrm{C}_{18} \mathrm{H}_{28} \mathrm{NaO}_{2} \mathrm{~S}[\mathrm{M}+\mathrm{Na}]^{+} 331.1708$, found 331.1705 . Anal. Calcd for $\mathrm{C}_{18} \mathrm{H}_{28} \mathrm{O}_{2} \mathrm{~S}$ : C, 70.08; H, 9.15. Found: C, 70.31; H, 9.09.

\section{Conformationally-Biased Model}
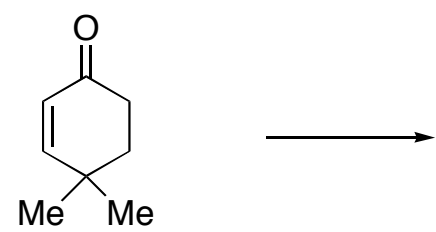<smiles>CC1(C)CCC(=O)CC1</smiles>

S-2 
4,4-Dimethylcyclohexanone S-2. ${ }^{9}$ To a solution of 4,4-dimethylcyclohex-2-enone (4.72 g, $\left.38.0 \mathrm{mmol}\right)$ in $20 \mathrm{~mL}$ of pentane was added $0.094 \mathrm{~g}$ of $10 \%$ palladium on carbon. The reaction mixture was stirred under hydrogen gas (40-50 psi) for $24 \mathrm{~h}$. The mixture was filtered through a Celite pad, rinsed with pentane $(3 \times 10$ $\mathrm{mL}$ ), and concentrated in vacuo to provide a white solid. ${ }^{1} \mathrm{H}$ NMR spectroscopic analysis of the unpurified product indicated $98 \%$ purity $(3.98 \mathrm{~g}, 83 \%)$. The spectral data correlates with the previously reported data for S-2: ${ }^{9,10}{ }^{1} \mathrm{H}$ NMR $\left(500 \mathrm{MHz}, \mathrm{CDCl}_{3}\right) \square 2.34(\mathrm{t}, J=6.9 \mathrm{~Hz}, 4 \mathrm{H}), 1.67(\mathrm{t}, J=6.9 \mathrm{~Hz}, 4 \mathrm{H}), 1.10(\mathrm{~s}, 6 \mathrm{H}) ;{ }^{13} \mathrm{C} \mathrm{NMR}$ $\left(125 \mathrm{MHz}, \mathrm{CDCl}_{3}\right) \square 212.9,39.4,38.3,30.2,27.8$.

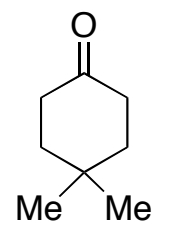

S-2<smiles>CC1(C)CCC(=O)C([SbH2]c2ccccc2)C1</smiles>

S-3

4,4-Dimethyl-2-phenylsulfanylcyclohexanone S-3.,11 The standard phenylsulfenylation procedure was followed with 4,4-dimethylcyclohexanone $\mathbf{S}-2(0.200 \mathrm{~g}, 1.58 \mathrm{mmol})$ in $2 \mathrm{~mL}$ of THF with LDA (1.90 mmol) and diphenyl disulfide $(0.380 \mathrm{~g}, 17.4 \mathrm{mmol})$. Purification of the resultant residue by silica gel chromatography (0:100 to 5:95 EtOAc:hexanes) yielded product $\mathbf{S - 3}$ as a yellow oil $(0.160 \mathrm{~g}, 43 \%)$. The spectral data correlates with the previously reported data for $\mathbf{S - 3} .{ }^{11}{ }^{1} \mathrm{H}$ NMR $\left(500 \mathrm{MHz}, \mathrm{CDCl}_{3}\right) \square 7.50(\mathrm{~m}, 2 \mathrm{H}), 7.24(\mathrm{~m}, 3 \mathrm{H}), 4.04$ (dd, $J=12.0,6.0 \mathrm{~Hz}, 1 \mathrm{H}), 2.03(\mathrm{ddd}, J=13.5,6.0,2.7 \mathrm{~Hz}, 1 \mathrm{H}), 2.54(\mathrm{~m}, 2 \mathrm{H}), 1.75(\mathrm{~m}, 3 \mathrm{H}), 1.17(\mathrm{~s}, 3 \mathrm{H}), 1.04(\mathrm{~s}$, $3 \mathrm{H}) ;{ }^{13} \mathrm{C}$ NMR $\left(125 \mathrm{MHz}, \mathrm{CDCl}_{3}\right) \square 207.4,132.1,129.2,127.4,54.6,47.6,39.9,37.9,30.9,25.3$; HRMS (ESI) $\mathrm{m} / \mathrm{z}$ calcd for $\mathrm{C}_{14} \mathrm{H}_{19} \mathrm{OS}[\mathrm{M}+\mathrm{H}]^{+} 235.1157$, found 235.1150 .<smiles>CC1(C)CCC(=O)[C@H](Sc2ccccc2)C1</smiles>

S-3

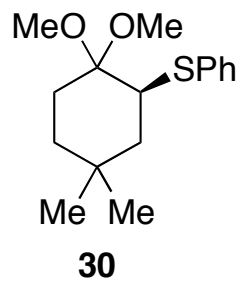

30

Acetal 30. The standard acetalization procedure was followed with $\mathbf{S - 3}(0.160 \mathrm{~g}, 0.68 \mathrm{mmol})$ in $2.2 \mathrm{~mL}$ of $\mathrm{MeOH}$ with trimethyl orthoformate $(0.373 \mathrm{~mL}, 3.41 \mathrm{mmol})$ and $\mathrm{H}_{2} \mathrm{SO}_{4}$ (3 drops). Purification of the resultant residue by silica gel chromatography (5:95 EtOAc:hexanes) yielded product $\mathbf{3 0}$ as a yellow oil (0.147 g, 77\%): ${ }^{1} \mathrm{H}\left(500 \mathrm{MHz}, \mathrm{CDCl}_{3}\right) \square 7.40(\mathrm{~m}, 2 \mathrm{H}), 7.26(\mathrm{~m}, 2 \mathrm{H}), 7.18(\mathrm{~m}, 1 \mathrm{H}), 3.50(\mathrm{dd}, J=9.4,4.6 \mathrm{~Hz}, 1 \mathrm{H}), 3.35$ (s, 3H), $3.30(\mathrm{~s}, 3 \mathrm{H}), 1.93(\mathrm{ddd}, J=13.7,7.2,3.8 \mathrm{~Hz}, 1 \mathrm{H}), 1.70(\mathrm{~m}, 3 \mathrm{H}), 1.41(\mathrm{ddd}, J=13.6,10.2,3.8 \mathrm{~Hz}, 1 \mathrm{H})$, $1.31(\mathrm{~m}, 1 \mathrm{H}), 1.01(\mathrm{~s}, 3 \mathrm{H}), 0.90(\mathrm{~s}, 3 \mathrm{H}) ;{ }^{13} \mathrm{C}\left(125 \mathrm{MHz}, \mathrm{CDCl}_{3}\right) \square 137.0,130.7,129.1,126.4,101.3,50.9,50.0$, 49.2, 43.7, 35.7, 31.4, 30.8, 27.7, 27.4; IR (thin film) 2952, 1584, $1439 \mathrm{~cm}^{-1}$; HRMS (ESI) $\mathrm{m} / z$ calcd for $\mathrm{C}_{16} \mathrm{H}_{24} \mathrm{NaO}_{2} \mathrm{~S}[\mathrm{M}+\mathrm{Na}]^{+}$303.1395, found 303.1403. Anal. Calcd for $\mathrm{C}_{14} \mathrm{H}_{18} \mathrm{OS}$ : C, 68.53; H, 8.63. Found: C, $68.35 ; \mathrm{H}, 8.37$.

\section{Sterically Encumbering Models}


<smiles>CC1(C)CC(=O)CC(C)(C)C1</smiles>

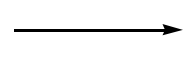

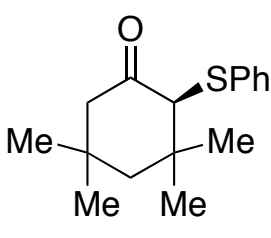

37

3,3,5,5-Tetramethyl-2-phenylsulfanylcyclohexanone $\quad \mathbf{3 7 .}^{12}$ The standard phenylsulfenylation procedure was followed with 3,3,5,5-tetramethylcyclohexanone $(5.00 \mathrm{~g}, 32.4 \mathrm{mmol})$ in $78 \mathrm{~mL}$ of THF with LDA (39.0 mmol) and diphenyl disulfide (7.78 g, $35.6 \mathrm{mmol})$. Purifcation of the resultant residue by silica gel chromatography (0:100 to 5:95 EtOAc:hexanes) yielded product $\mathbf{3 7}$ as a yellow oil (6.54 g, 77\%). The spectral data correlates with the previously reported data for 37. ${ }^{12}{ }^{1} \mathrm{H} \mathrm{NMR}\left(400 \mathrm{MHz}, \mathrm{CDCl}_{3}\right) \square 7.36(\mathrm{~m}, 2 \mathrm{H}), 7.23(\mathrm{~m}$, $3 \mathrm{H}), 3.50(\mathrm{~s}, 1 \mathrm{H}), 2.89(\mathrm{~d}, J=16.3 \mathrm{~Hz}, 1 \mathrm{H}), 2.04(\mathrm{~d}, J=16.3 \mathrm{~Hz}, 1 \mathrm{H}), 1.83(\mathrm{~d}, J=17.2 \mathrm{~Hz}, 1 \mathrm{H}), 1.50(\mathrm{~d}, J=$ $17.1 \mathrm{~Hz}, 1 \mathrm{H}), 1.19(\mathrm{~s}, 3 \mathrm{H}), 1.16(\mathrm{~s}, 3 \mathrm{H}), 1.07(\mathrm{~s}, 3 \mathrm{H}), 1.05(\mathrm{~s}, 3 \mathrm{H}) ;{ }^{13} \mathrm{C} \mathrm{NMR}\left(125 \mathrm{MHz}, \mathrm{CDCl}_{3}\right) \square 208.5,135.2$, 131.6, 129.3 127.4, 67.4, 50.7, 49.8, 40.0, 36.1, 32.7, 31.0, 30.9, 27.9; HRMS (CI) $\mathrm{m} / z$ calcd for $\mathrm{C}_{16} \mathrm{H}_{23} \mathrm{OS}[\mathrm{M}+$ $\mathrm{H}]^{+} 263.1470$, found 263.1461 .

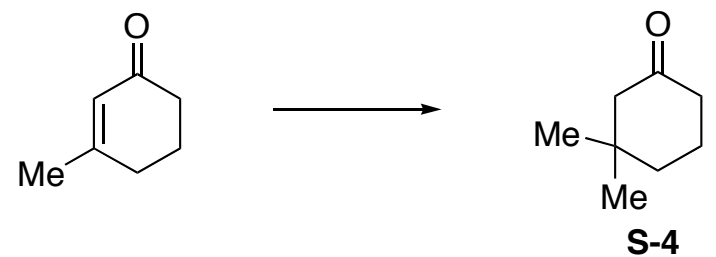

3,3-Dimethylcyclohexanone $\mathbf{S - 4} .^{13}$ A solution of CuI (8.64 g, $\left.45.4 \mathrm{mmol}\right)$ in THF (90 mL) was cooled to $-78{ }^{\circ} \mathrm{C}$ and methyllithium $(56.8 \mathrm{~mL}, 90.8 \mathrm{mmol})$ was added dropwise. The mixture was allowed to warm until a homogeneous, colorless solution resulted. The solution was cooled to $-78{ }^{\circ} \mathrm{C}$ and $\mathrm{BF}_{3} \mathrm{OEt}_{2}(11.40 \mathrm{~mL}$, $90.8 \mathrm{mmol}$ ) was added dropwise. The substrate, 3-methyl-2-cyclohexenone (5.00 g, $45.4 \mathrm{mmol})$ was added and the yellow reaction mixture was stirred for $2.5 \mathrm{~h}$ at ambient temperature. The reaction mixture was diluted with $30 \mathrm{~mL}$ of a $10 \% \mathrm{NH}_{4} \mathrm{OH} / 90 \%$ saturated $\mathrm{NH}_{4} \mathrm{Cl}$ solution and then was poured into a separatory funnel with 30 $\mathrm{mL}$ of ether. The organic layer was separated from the aqueous layer and rinsed with a saturated sodium chloride solution $(30 \mathrm{~mL})$, dried $\left(\mathrm{Na}_{2} \mathrm{SO}_{4}\right)$, and concentrated in vacuo: Purification ${ }^{14}$ of the resultant residue by vacuum distillation $\left(30-32{ }^{\circ} \mathrm{C}\right.$ at $\left.48 \mathrm{~mm} \mathrm{Hg}\right)$ yielded product $\mathbf{S}-4$ (3.56 g, 61\%) as a colorless liquid. The spectral data correlates with the previously reported data for $\mathbf{S}-\mathbf{4} .{ }^{15}{ }^{1} \mathrm{H} \mathrm{NMR}\left(500 \mathrm{MHz}, \mathrm{CDCl}_{3}\right) \square 2.26(\mathrm{t}, J=$ $6.8 \mathrm{~Hz}, 2 \mathrm{H}), 2.17(\mathrm{~s}, 2 \mathrm{H}), 1.88(\mathrm{dt}, J=6.5 \mathrm{~Hz}, 2 \mathrm{H}), 1.58(\mathrm{t}, J=6.1 \mathrm{~Hz}, 2 \mathrm{H}), 0.97(\mathrm{~s}, 6 \mathrm{H}) ;{ }^{13} \mathrm{C}(125 \mathrm{MHz}$ $\left.\mathrm{CDCl}_{3}\right) \square 212.5,55.3,41.1,38.3,36.4,28.9,22.8$.<smiles>CC1(C)CCCC(=O)C1</smiles>

S-4<smiles>CC1(C)CCC(Sc2ccccc2)C(=O)C1</smiles>

S-5

5,5-Dimethyl-2-phenylsulfanylcyclohexanone S-5. ${ }^{15}$ The standard phenylsulfenylation procedure was followed with S-4 (2.14 g, $16.6 \mathrm{mmol})$ in $34 \mathrm{~mL}$ of THF with LDA (19.88 mmol) and diphenyl disulfide (3.97 g, $18.2 \mathrm{mmol})$. Purification of the resultant residue by silica gel chromatography (0:100 to 5:95 EtOAC:hexanes) the yielded product $\mathbf{S - 5}$ as a yellow oil $(2.54 \mathrm{~g}, 65 \%)$. The spectral data correlates with the 
previously reported data for $\mathbf{S - 5}:{ }^{15}{ }^{1} \mathrm{H}$ NMR $\left(400 \mathrm{MHz}, \mathrm{CDCl}_{3}\right) \square 7.20-7.40(\mathrm{~m}, 5 \mathrm{H}), 3.77(\mathrm{~m}, 1 \mathrm{H}), 2.75(\mathrm{~d}, J=$ $13.2 \mathrm{~Hz}, 1 \mathrm{H}), 2.43(\mathrm{~m}, 1 \mathrm{H}), 2.08(\mathrm{dt}, J=12.9,1.3 \mathrm{~Hz}, 1 \mathrm{H}), 2.01(\mathrm{~m}, 1 \mathrm{H}), 1.81(\mathrm{ddd}, J=13.7,9.5,4.1 \mathrm{~Hz}, 1 \mathrm{H})$, $1.52(\mathrm{~m}, 1 \mathrm{H}), 1.00(\mathrm{~s}, 3 \mathrm{H}), 0.93(\mathrm{~s}, 3 \mathrm{H}) ;{ }^{13} \mathrm{C}$ NMR $\left(125 \mathrm{MHz}, \mathrm{CDCl}_{3}\right) \square 207.8,133.9,132.1,129.3,127.7,55.6$, 52.2, 37.0, 35.9, 29.8, 29.3, 28.1; HRMS (CI) $\mathrm{m} / z$ calcd for $\mathrm{C}_{14} \mathrm{H}_{19} \mathrm{OS}[\mathrm{M}+\mathrm{H}]^{+} 235.1157$ found, 235.1159 .<smiles>CC1(C)CCC(Sc2ccccc2)C(=O)C1</smiles>

S-5

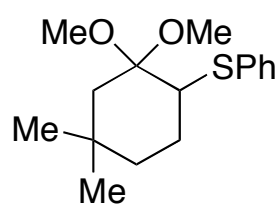

32

Acetal 32. The standard acetalization procedure was followed with $\mathbf{S - 5}(0.355 \mathrm{~g}, 1.51 \mathrm{mmol})$ in $15 \mathrm{~mL}$ of $\mathrm{MeOH}$ with trimethyl orthoformate $(1.20 \mathrm{~mL}, 10.6 \mathrm{mmol})$ and $\mathrm{H}_{2} \mathrm{SO}_{4}$ (3 drops). Purification of the resultant residue by silica gel chromatography (2:98 EtOAC:hexanes) yielded product 32 as a yellow oil $(0.380 \mathrm{~g}, 89 \%)$ : ${ }^{1} \mathrm{H}$ NMR (400 MHz, $\left.\mathrm{CDCl}_{3}\right) \square 7.19-7.45$ (m, 5H), 3.62 (broad s, 1H), 3.26 (s, 3H), 3.21 (s, 3H), 1.93 (dddd, $J=$ 12.5, 12.0, 3.5, 3.3 Hz, 1H), 1.81 (ddd, $J=13.3,13.0,3.2 \mathrm{~Hz}, 1 \mathrm{H}), 1.68(\mathrm{dt}, J=14.6,1.8 \mathrm{~Hz}, 1 \mathrm{H}), 1.57(\mathrm{~m}$, $1 \mathrm{H}), 1.48(\mathrm{~d}, J=14.6 \mathrm{~Hz}, 1 \mathrm{H}), 1.06(\mathrm{~m}, 1 \mathrm{H}), 1.02,(\mathrm{~s}, 3 \mathrm{H}), 0.97(\mathrm{~s}, 3 \mathrm{H}) ;{ }^{13} \mathrm{C}$ NMR $\left(125 \mathrm{MHz}, \mathrm{CDCl}_{3}\right) \square 135.7$, 132.0, 129.2, 126.9, 101.7, 50.8, 48.7, 47.5, 40.1, 33.6, 33.5, 31.6, 26.5, 24.2; IR (thin film) 3059, 2938, 2829 $\mathrm{cm}^{-1}$; HRMS (CI) $\mathrm{m} / z$ calcd for $\mathrm{C}_{15} \mathrm{H}_{20} \mathrm{OS}[\mathrm{M}-\mathrm{MeOH}]^{+} 249.1313$, found 249.1309. Anal. Calcd for $\mathrm{C}_{16} \mathrm{H}_{24} \mathrm{O}_{2} \mathrm{~S}$ : C, $68.53 ; \mathrm{H}, 8.63$. Found: C, 68.78; H, 8.78.

\section{Synthesis of 2-Heteroatom Substituted Cyclohexanone Acetals}

\section{A. Fluoro acetal 11a}
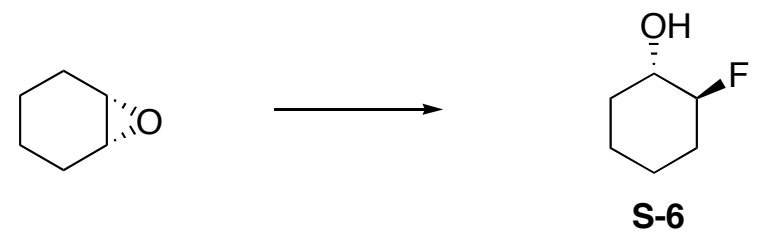

trans-Fluorocyclohexanol S-6. ${ }^{16-18}$ To cyclohexene oxide $(7.00 \mathrm{~g}, 71.3 \mathrm{mmol})$ was added $\mathrm{Et}_{3} \mathrm{~N} \cdot 3 \mathrm{HF}$ $\left(11.60 \mathrm{~mL}, 71.2 \mathrm{mmol}\right.$ ) and the reaction mixture was heated to $115{ }^{\circ} \mathrm{C}$ for $6 \mathrm{~h}$. After cooling to $22{ }^{\circ} \mathrm{C}$, the reaction mixture was poured over ice, treated with $20 \mathrm{~mL}$ of $\mathrm{NH}_{4} \mathrm{OH}$, and extracted with $20 \mathrm{~mL}$ of $\mathrm{CH}_{2} \mathrm{Cl}_{2}$. The organic layer was washed with $15 \% \mathrm{HCl}$ solution $(3 \times 15 \mathrm{~mL})$, a saturated sodium chloride solution $(20 \mathrm{~mL})$, dried $\left(\mathrm{Na}_{2} \mathrm{SO}_{4}\right)$, and concentrated in vacuo. Purification of the resultant residue by fractional distillation (90-95 ${ }^{\circ} \mathrm{C}, 10 \mathrm{~mm} \mathrm{Hg}$ ) yielded the product $\mathbf{S}-6$ as a pale yellow liquid $(5.20 \mathrm{~g}, 62 \%)$. The spectral data correlates with the previously reported data for $\mathbf{S - 6 :}:{ }^{17,18}{ }^{1} \mathrm{H}$ NMR $\left(500 \mathrm{MHz}, \mathrm{CDCl}_{3}\right) \square 4.22$ (dddd, $J=51.6,10.7,8.4,4.8 \mathrm{~Hz}$, $1 \mathrm{H}), 3.63(\mathrm{~m}, 1 \mathrm{H}), 2.63(\mathrm{~m}, 1 \mathrm{H}), 2.08(\mathrm{~m}, 2 \mathrm{H}), 1.69(\mathrm{~m}, 2 \mathrm{H}), 1.43(\mathrm{~m}, 1 \mathrm{H}), 1.28(\mathrm{~m}, 3 \mathrm{H}) ;{ }^{13} \mathrm{C} \mathrm{NMR}(500 \mathrm{MHz}$, $\left.\mathrm{CDCl}_{3}\right) \square 96.4(\mathrm{~d}, J=172.9 \mathrm{~Hz}), 73.5(\mathrm{~d}, J=17.8 \mathrm{~Hz}), 31.9(\mathrm{~d}, J=7.6 \mathrm{~Hz}), 30.5(\mathrm{~d}, J=15.3 \mathrm{~Hz}), 23.8,23.7(\mathrm{~d}$, $J=5.1 \mathrm{~Hz}$ ); HRMS (ESI) $\mathrm{m} / z$ calcd for $\mathrm{C}_{6} \mathrm{H}_{11} \mathrm{FNaO}[\mathrm{M}+\mathrm{Na}]^{+} 141.0692$, found 141.0687.

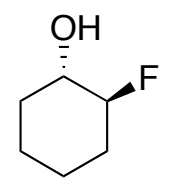

S-6<smiles>O=C1CCCCC1F</smiles>

17 
2-Fluorocyclohexanone $17 .^{18}$ To a cooled solution $\left(0{ }^{\circ} \mathrm{C}\right)$ of trans-fluorocyclohexanol S-6 $(1.00 \mathrm{~g}, 8.50$ $\mathrm{mmol})$ in $10 \mathrm{~mL}$ of acetone was added $\mathrm{CrO}_{3}(1.28 \mathrm{~g}, 12.8 \mathrm{mmol})$. The reaction mixture was allowed to warm to $22{ }^{\circ} \mathrm{C}$. After stirring for $24 \mathrm{~h}$, the reaction mixture was diluted with ether $(20 \mathrm{~mL})$ and treated with $\mathrm{NaHCO}_{3}(20$ $\mathrm{mL}$ ). The organic layer was washed with $\mathrm{H}_{2} \mathrm{O}$ until a clear, yellow solution resulted. This solution was rinsed with a saturated sodium chloride solution $(20 \mathrm{~mL})$, dried $\left(\mathrm{Na}_{2} \mathrm{SO}_{4}\right)$, and concentrated in vacuo. ${ }^{1} \mathrm{H}$ NMR spectroscopic analysis of the unpurified product indicated $95 \%$ purity of the yellow oil $(0.560 \mathrm{~g}, 57 \%)$. The spectral data correlates with the previously reported data for 17: ${ }^{18} \mathrm{H}$ NMR $\left(400 \mathrm{MHz}, \mathrm{CDCl}_{3}\right) \square 4.88$ (dddd, $J=$ 48.9, 11.3, 6.2, $1.1 \mathrm{~Hz}, 1 \mathrm{H}), 2.55(\mathrm{~m}, 1 \mathrm{H}), 2.41(\mathrm{~m}, 1 \mathrm{H}), 2.33(\mathrm{~m}, 1 \mathrm{H}), 1.98(\mathrm{~m}, 2 \mathrm{H}), 1.86(\mathrm{~m}, 1 \mathrm{H}), 1.68(\mathrm{~m}$, $2 \mathrm{H}) ;{ }^{13} \mathrm{C}$ NMR $\left(125 \mathrm{MHz}, \mathrm{CDCl}_{3}\right) \square 208.2,93.1(\mathrm{~d}, J=189.3 \mathrm{~Hz}), 40.5,34.6(\mathrm{~d}, J=18.5 \mathrm{~Hz}), 27.2,23.0(\mathrm{~d}, J=$ $9.6 \mathrm{~Hz}$ ); HRMS (ESI) $\mathrm{m} / z$ calcd for $\mathrm{C}_{6} \mathrm{H}_{9} \mathrm{FNaO}[\mathrm{M}+\mathrm{Na}]^{+} 139.0535$, found 139.0542 .

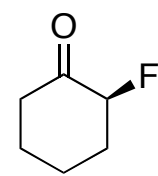

17

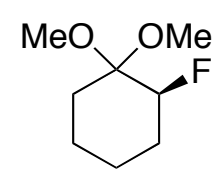

$11 a$

Fluoro acetal 11a. The standard acetalization procedure was followed with 2-fluorocyclohexanone $17(1.81 \mathrm{~g}, 15.7 \mathrm{mmol})$ in $50 \mathrm{~mL}$ of $\mathrm{MeOH}$ with trimethyl orthoformate $(14.0 \mathrm{~mL}, 94.7 \mathrm{mmol})$ and $\mathrm{H}_{2} \mathrm{SO}_{4}(3$ drops). Purification of the resultant residue by silica gel chromatography (0:100 to 3:97 EtOAc:hexanes) yielded the product 11a as a pale yellow oil $(0.960 \mathrm{~g}, 38 \%)$ : ${ }^{1} \mathrm{H}$ NMR $\left(500 \mathrm{MHz}, \mathrm{CDCl}_{3}\right) \square 4.55(\mathrm{dt}, J=48.9$, 2.1, 1H), $3.25(\mathrm{~d}, J=1.7 \mathrm{~Hz}, 3 \mathrm{H}), 3.19(\mathrm{~d}, J=1.7 \mathrm{~Hz}, 3 \mathrm{H}), 1.94(\mathrm{~m}, 1 \mathrm{H}), 1.68(\mathrm{~m}, 3 \mathrm{H}), 1.52(\mathrm{~m}, 2 \mathrm{H}), 1.42(\mathrm{~m}$, $1 \mathrm{H}), 1.34(\mathrm{~m}, 1 \mathrm{H}) ;{ }^{13} \mathrm{C}$ NMR $\left(125 \mathrm{MHz}, \mathrm{CDCl}_{3}\right) \square 98.9(J=21.5 \mathrm{~Hz}), 88.2(\mathrm{~d}, J=175.3 \mathrm{~Hz}), 47.8,28.7,28.4(J$ $=13.3 \mathrm{~Hz}$ ), 21.8, $20.0\left(\mathrm{~J}=2.1 \mathrm{~Hz}\right.$ ); IR (thin film) $2927,1063 \mathrm{~cm}^{-1}$; HRMS (ESI) $\mathrm{m} / z$ calcd for $\mathrm{C}_{8} \mathrm{H}_{15} \mathrm{FNaO}_{2}[\mathrm{M}$ $+\mathrm{Na}^{+}$185.0954, found 185.0947. Anal. Calcd for $\mathrm{C}_{8} \mathrm{H}_{15} \mathrm{O}_{2} \mathrm{~F}: \mathrm{C}, 59.24 ; \mathrm{H}, 9.32$. Found: C, 59.54; H, 9.30.

\section{B. Chloro acetal 11b}<smiles>O=C1CCCCC1Cl</smiles><smiles>COC1(OC)CCCCC1Cl</smiles>

11b

Chloro acetal 11b. ${ }^{19}$ The standard acetalization procedure was followed with 2-chlorocyclohexanone (3.48 g, $26.2 \mathrm{mmol})$ in $90 \mathrm{~mL}$ of $\mathrm{MeOH}$ with trimethyl orthoformate $(19.0 \mathrm{~mL}, 131.0 \mathrm{mmol})$ and $\mathrm{H}_{2} \mathrm{SO}_{4}(3$ drops). Purification of the resultant residue by vacuum distillation $\left(50{ }^{\circ} \mathrm{C}\right.$ at $\left.10 \mathrm{~mm} \mathrm{Hg}\right)$ yielded the product 11b as a colorless oil $(3.41 \mathrm{~g}, 73 \%)$. The spectral data correlates with the previously reported data for $\mathbf{1 1 b}:{ }^{19}{ }^{1} \mathrm{H}$ NMR (400 MHz, $\left.\mathrm{CDCl}_{3}\right) \square 4.20(\mathrm{~m}, 1 \mathrm{H}), 3.17(\mathrm{~s}, 3 \mathrm{H}), 3.25(\mathrm{~s}, 3 \mathrm{H}), 2.01(\mathrm{~m}, 1 \mathrm{H}), 1.89(\mathrm{~m}, 1 \mathrm{H}), 1.77(\mathrm{~m}, 2 \mathrm{H})$, $1.69(\mathrm{~m}, 1 \mathrm{H}), 1.57(\mathrm{~m}, 1 \mathrm{H}), 1.46(\mathrm{~m}, 1 \mathrm{H}), 1.31(\mathrm{~m}, 1 \mathrm{H}) ;{ }^{13} \mathrm{C} \mathrm{NMR}\left(125 \mathrm{MHz}, \mathrm{CDCl}_{3}\right) \square 100.5,59.5,48.4,47.3$, 31.5, 27.0, 22.1, 19.6 .

\section{Iodo acetal 11c}




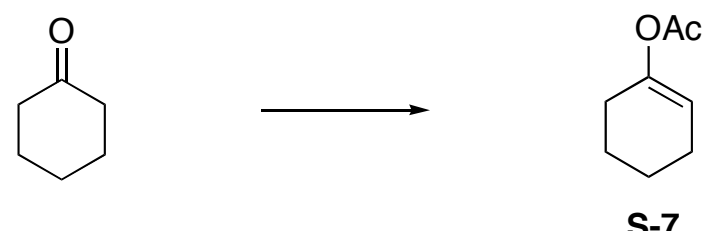

Enol acetate S-7. ${ }^{20}$ A solution of cyclohexanone $(9.50 \mathrm{~g}, 96.5 \mathrm{mmol})$ and isopropenyl acetate $(27.0 \mathrm{~g}$, $289 \mathrm{mmol})$ was treated with $p$-TsOH $(0.18 \mathrm{~g}, 0.96 \mathrm{mmol})$. The reaction mixture was heated to reflux $\left(90{ }^{\circ} \mathrm{C}\right)$ and allowed to stir for $13 \mathrm{~h}$. After cooling to $22{ }^{\circ} \mathrm{C}$, the reaction mixture was concentrated in vacuo. Purification of the brown residue by vacuum distillation $\left(89-92{ }^{\circ} \mathrm{C}\right.$ at $48 \mathrm{~mm} \mathrm{Hg}$ ) yielded the product $\mathbf{S - 7}$ as a colorless liquid $(10.10 \mathrm{~g}, 75 \%)$. The spectral data correlates with the previously reported data for S-7. ${ }^{20}$<smiles>CC(=O)OC1=CCCCC1</smiles>

S-7<smiles>COC1(OC)CCCCC1I</smiles>

$11 \mathrm{c}$

Iodo acetal 11c. ${ }^{21,22}$ A solution of enol acetate $\mathbf{S - 7}(0.500 \mathrm{~g}, 3.57 \mathrm{mmol})$ in $10 \mathrm{~mL}$ of $\mathrm{MeOH}$ was treated with $N$-iodosuccinimide $(0.88 \mathrm{~g}, 3.92 \mathrm{mmol})$. The reaction vessel was covered with aluminum foil to avoid light. After stirring at $22{ }^{\circ} \mathrm{C}$ for $12 \mathrm{~h}$, the brownish reaction mixture was concentrated in vacuo. Purification of the resultant residue by silica gel chromatography (0:100 to 2:98 EtOAc:hexanes) yielded the product 11c as a pale yellow oil $(0.693 \mathrm{~g}, 72 \%)$. The spectral data correlates with the previously reported data for 11c: ${ }^{21,22}{ }^{1} \mathrm{H}$ $\operatorname{NMR}\left(500 \mathrm{MHz}, \mathrm{CDCl}_{3}\right) \square 4.51(\mathrm{~m}, 1 \mathrm{H}), 3.18(\mathrm{~s}, 6 \mathrm{H}), 1.94(\mathrm{~m}, 3 \mathrm{H}), 1.81(\mathrm{~m}, 1 \mathrm{H}), 1.66(\mathrm{~m}, 1 \mathrm{H}), 1.53(\mathrm{~m}, 2 \mathrm{H})$, $1.40(\mathrm{~m}, 1 \mathrm{H}) ;{ }^{13} \mathrm{C} \mathrm{NMR}\left(125 \mathrm{MHz}, \mathrm{CDCl}_{3}\right) \square 99.7,48.9,47.1,36.3,33.7,28.0,22.4,22.2 ; \mathrm{HRMS}(\mathrm{ESI}) \mathrm{m} / \mathrm{z}$ calcd for $\mathrm{C}_{8} \mathrm{H}_{15} \mathrm{INaO}_{2}[\mathrm{M}+\mathrm{Na}]^{+} 293.0015$, found 293.0019 .

\section{Phenoxy Acetal 11d}<smiles>C1CC[C@H]2O[C@H]2C1</smiles>

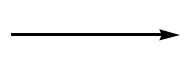<smiles>OC1CCCCC1Oc1ccccc1</smiles>

\section{S-8}

2-Phenoxycyclohexanol S-8. ${ }^{23}$ To a heterogeneous mixture of $\mathrm{NaOH}(6.00 \mathrm{~g}, 150 \mathrm{mmol})$ in $40 \mathrm{~mL}$ of $\mathrm{H}_{2} \mathrm{O}$ was added phenol $(14.10 \mathrm{~g}, 150 \mathrm{mmol})$. The reaction mixture was heated to reflux $\left(105{ }^{\circ} \mathrm{C}\right)$ followed by addition of cyclohexene oxide $(5.00 \mathrm{~mL}, 50 \mathrm{mmol})$. After stirring for $3 \mathrm{~h}$ at reflux, the white heterogeneous reaction mixture was filtered. The white precipitate was allowed to dry under vacuum for $8 \mathrm{~h}$. Purification of the resultant white solid was achieved by recrystallization from hexane to afford $\mathbf{S - 8}(6.13 \mathrm{~g}, 65 \%)$. The spectral data correlates with the previously reported data for $\mathbf{S - 8} .^{23}$<smiles>O[C@H]1CCCC[C@H]1Oc1ccccc1</smiles>

S-8<smiles>O=C1CCCC[C@H]1Oc1ccccc1</smiles>

S-9 
2-Phenoxycyclohexanone S-9. ${ }^{24}$ To a cooled $\left(-60{ }^{\circ} \mathrm{C}\right)$ solution of oxalyl chloride ${ }^{25}(0.735 \mathrm{~mL}, 8.60$ mmol) in $11 \mathrm{~mL}$ of $\mathrm{CH}_{2} \mathrm{Cl}_{2}$ was added DMSO $(1.28 \mathrm{~mL}, 18.0 \mathrm{mmol})$, followed by slow addition of 2phenoxycyclohexanol S-8 $(1.50 \mathrm{~g}, 7.80 \mathrm{mmol})$. After $30 \mathrm{~min}$ at $-60{ }^{\circ} \mathrm{C}$, the reaction mixture was treated with $\mathrm{Et}_{3} \mathrm{~N}(4.15 \mathrm{~mL}, 29.8 \mathrm{mmol})$. After stirring for $5 \mathrm{~min}$, the reaction mixture was warmed slowly to $22^{\circ} \mathrm{C}$ and treated with $\mathrm{H}_{2} \mathrm{O}(5 \mathrm{~mL})$ and diluted with $5 \mathrm{~mL}$ of $\mathrm{CH}_{2} \mathrm{Cl}_{2}$. The reaction mixture was poured into a separatory funnel and the organic layer was washed with $\mathrm{H}_{2} \mathrm{O}(3 \times 5 \mathrm{~mL}), 1 \% \mathrm{HCl}(5 \mathrm{~mL})$, and $1.0 \mathrm{M} \mathrm{Na}_{2} \mathrm{CO}_{3}(5 \mathrm{~mL})$. The organic layer was dried $\left(\mathrm{Na}_{2} \mathrm{SO}_{4}\right)$ and concentrated in vacuo to provide a white solid. ${ }^{1} \mathrm{H}$ NMR spectroscopic analysis of the unpurified product indicated $98 \%$ purity of the white solid $(1.41 \mathrm{~g}, 95 \%)$. The spectral data correlates with the previously reported data for S-9. ${ }^{24}{ }^{1} \mathrm{H}$ NMR $\left(500 \mathrm{MHz}, \mathrm{CDCl}_{3}\right) \square 7.25(\mathrm{~m}, 2 \mathrm{H}), 6.95(\mathrm{~m}$, 1H), $6.85(\mathrm{~m}, 2 \mathrm{H}), 4.63(\mathrm{ddd}, J=9.5,5.2,1.0 \mathrm{~Hz}, 1 \mathrm{H}), 2.61(\mathrm{~m}, 1 \mathrm{H}), 2.33(\mathrm{~m}, 2 \mathrm{H}), 2.05(\mathrm{~m}, 3 \mathrm{H}), 1.77(\mathrm{~m}, 2 \mathrm{H})$; ${ }^{13} \mathrm{C}$ NMR $\left(125 \mathrm{MHz}, \mathrm{CDCl}_{3}\right) \square 208.6,157.9,129.7,121.7,115.8,80.9,40.8,34.7,28.1,23.1 ; \mathrm{HRMS}(\mathrm{ESI}) \mathrm{m} / z$ calcd for $\mathrm{C}_{12} \mathrm{H}_{14} \mathrm{NaO}_{2}[\mathrm{M}+\mathrm{Na}]^{+} 213.0892$, found 213.0887 .<smiles>O=C1CCCCC1Oc1ccccc1</smiles>

S-9

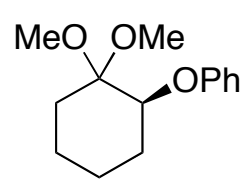

11d

Phenoxy acetal 11d. The standard acetalization procedure was followed with 2-phenoxycyclohexanone S-9 $(0.363 \mathrm{~g}, 1.92 \mathrm{mmol})$ in $19 \mathrm{~mL}$ of $\mathrm{MeOH}$ with trimethyl orthoformate $(1.05 \mathrm{~mL}, 9.60 \mathrm{mmol}) \mathrm{and}_{2} \mathrm{SO}_{4}(3$ drops). Purification of the resultant residue by silica gel chromatography (3:97 EtOAc:hexanes) yielded product 11d as a colorless oil $(0.44 \mathrm{~g}, 97 \%):{ }^{1} \mathrm{H}$ NMR $\left(500 \mathrm{MHz}, \mathrm{CDCl}_{3}\right) \square 7.26(\mathrm{~m}, 2 \mathrm{H}), 6.94(\mathrm{~m}, 3 \mathrm{H}), 4.44(\mathrm{t}$, $J=3.1 \mathrm{~Hz}, 1 \mathrm{H}), 3.24(\mathrm{~s}, 3 \mathrm{H}), 3.17(\mathrm{~s}, 3 \mathrm{H}), 1.96(\mathrm{~m}, 1 \mathrm{H}), 1.85(\mathrm{~m}, 2 \mathrm{H}), 1.67(\mathrm{~m}, 1 \mathrm{H}), 1.59(\mathrm{~m}, 1 \mathrm{H}), 1.53(\mathrm{~m}$, $1 \mathrm{H}), 1.38(\mathrm{~m}, 2 \mathrm{H}) ;{ }^{13} \mathrm{C} \mathrm{NMR}\left(125 \mathrm{MHz}, \mathrm{CDCl}_{3}\right) \square 157.8,129.7,121.2,116.4,100.3,73.7,47.9,47.8,28.5$, 26.5, 22.2, 20.1; IR (thin film) 3040, 2862, $1240 \mathrm{~cm}^{-1}$; HRMS (CI) m/z calcd for $\mathrm{C}_{13} \mathrm{H}_{17} \mathrm{O}_{2}\left[\mathrm{M}-\mathrm{CH}_{3} \mathrm{O}\right]^{+}$ 205.1228, found 205.1222. Anal. Calcd for $\mathrm{C}_{14} \mathrm{H}_{20} \mathrm{O}_{3}: \mathrm{C}, 71.16 ; \mathrm{H}, 8.53$. Found: $\mathrm{C}, 71.26 ; \mathrm{H}, 8.61$.

\section{Nucleophilic Substitution Reactions}

General Procedure for Allylation of Acetals: A solution of acetal in $\mathrm{CH}_{2} \mathrm{Cl}_{2}(0.10 \mathrm{M})$ was treated with allyltrimethylsilane or 2-methylpropenyltrimethylsilane (4.0 equiv), then cooled to $-78{ }^{\circ} \mathrm{C}$. The appropriate Lewis acid (1.2 equiv, $1.0 \mathrm{M}$ in $\mathrm{CH}_{2} \mathrm{Cl}_{2}$ ) was added dropwise and the reaction mixture was allowed to warm to $22{ }^{\circ} \mathrm{C}$ for $24 \mathrm{~h}$. The reaction mixture was poured into a separatory funnel containing saturated aqueous $\mathrm{Na}_{2} \mathrm{HPO}_{4}\left(1 \mathrm{~mL}\right.$ per mmol acetal). The aqueous layer was extracted with three portions of $\mathrm{CH}_{2} \mathrm{Cl}_{2}(1$ $\mathrm{mL}$ per mmol acetal). The combined organic layers were washed with a saturated sodium chloride solution, dried $\left(\mathrm{Na}_{2} \mathrm{SO}_{4}\right)$ and concentrated in vacuo. The unpurified mixture was analyzed by GC and ${ }^{1} \mathrm{H} \mathrm{NMR}$ spectroscopy, then purified as indicated.

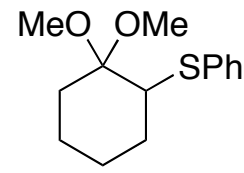

7

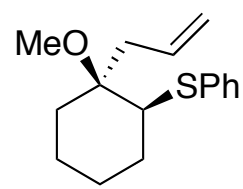

8 
Allyl Product 8. The standard allylation procedure was followed with acetal $7(0.11 \mathrm{~g}, 0.47 \mathrm{mmol})$, allyltrimethylsilane $(0.30 \mathrm{~mL}, 1.90 \mathrm{mmol})$, and $\mathrm{BF}_{3} \cdot \mathrm{OEt}_{2}(0.072 \mathrm{~mL}, 0.56 \mathrm{mmol})$. GC and ${ }^{1} \mathrm{H} \mathrm{NMR}$ spectroscopic analysis of the unpurified product detected only a single isomer. Purification of the resultant residue by silica gel chromatography (0:100 to 2:98 EtOAc:hexanes) yielded the product as a colorless oil (0.071 g, 61\%): ${ }^{1} \mathrm{H}$ NMR (500 MHz, $\left.\mathrm{CDCl}_{3}\right) \square 7.40(\mathrm{~m}, 2 \mathrm{H}), 7.26(\mathrm{t}, J=7.5 \mathrm{~Hz}, 2 \mathrm{H}), 7.18(\mathrm{~m}, 1 \mathrm{H}), 5.82(\mathrm{ddt}, J$ $=17.0,12.4,4.9 \mathrm{~Hz}, 1 \mathrm{H}), 5.16(\mathrm{dd}, J=17.0,1.8 \mathrm{~Hz}, 1 \mathrm{H}), 5.10(\mathrm{dd}, J=10.1,2.0 \mathrm{~Hz}, 1 \mathrm{H}), 3.25(\mathrm{~s}, 3 \mathrm{H}), 3.10$ $(\mathrm{dd}, J=11.1,4.1 \mathrm{~Hz}, 1 \mathrm{H}), 2.80(\mathrm{dd}, J=13.3,7.6 \mathrm{~Hz}, 1 \mathrm{H}), 2.41(\mathrm{dd}, J=13.3,7.3 \mathrm{~Hz}, 1 \mathrm{H}), 1.90(\mathrm{~m}, 2 \mathrm{H}), 1.78$ $(\mathrm{m}, 1 \mathrm{H}), 1.70(\mathrm{~m}, 1 \mathrm{H}), 1.47(\mathrm{~m}, 3 \mathrm{H}), 1.14(\mathrm{~m}, 1 \mathrm{H}) ;{ }^{13} \mathrm{C} \mathrm{NMR}\left(125 \mathrm{MHz}, \mathrm{CDCl}_{3}\right) \square$ 136.7, 134.0, 131.6, 129.1, 126.5, 118.9, 77.8, 54.7, 48.8, 40.3, 30.9, 30.0, 25.9, 21.4; IR (thin film) 3074, 2935, 2858, $1444 \mathrm{~cm}^{-1}$; HRMS (CI) $\mathrm{m} / z$ calcd for $\mathrm{C}_{16} \mathrm{H}_{22} \mathrm{OS}[\mathrm{M}]^{+}$262.1391, found 262.1393. Anal. Calcd for $\mathrm{C}_{16} \mathrm{H}_{22} \mathrm{OS}$ : C, 73.23; $\mathrm{H}, 8.45$. Found: C, 73.50; H, 8.68.

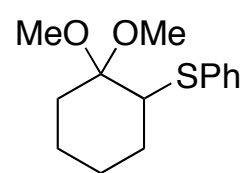

7

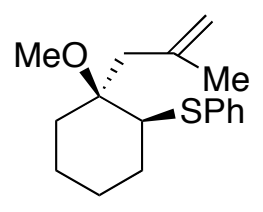

9

Methallyl Product 9. The standard allylation procedure was followed with acetal 7 (0.376 g, 1.50 mmol), 2-methylpropenyltrimethylsilane (1.02 mL, $5.96 \mathrm{mmol})$, and $\mathrm{BF}_{3} \cdot \mathrm{OEt}_{2}(0.225 \mathrm{~mL}, 1.80 \mathrm{mmol})$. GC and ${ }^{1} \mathrm{H}$ NMR spectroscopic analysis of the unpurified product detected only a single isomer was present. Purification by silica gel chromatography (0:100 to 2:98 EtOAc:hexanes) yielded the product as a colorless solid $(0.260 \mathrm{~g}, 63 \%)$. X-ray quality crystals were grown from a 3:1 mixture of $\mathrm{CHCl}_{3}$ and hexanes in which slow evaporation provided the crystal: $\mathrm{mp} 35-38{ }^{\circ} \mathrm{C} ;{ }^{1} \mathrm{H} \mathrm{NMR}\left(500 \mathrm{MHz}, \mathrm{CDCl}_{3}\right) \square 7.35(\mathrm{~d}, J=7.3 \mathrm{~Hz}, 2 \mathrm{H})$, $7.26(\mathrm{~m}, 2 \mathrm{H}), 7.16(\mathrm{t}, J=7.3 \mathrm{~Hz}, 1 \mathrm{H}), 4.91(\mathrm{~m}, 1 \mathrm{H}), 4.89(\mathrm{~m}, 1 \mathrm{H}), 3.30(\mathrm{dd}, J=4.2,10.1 \mathrm{~Hz}, 1 \mathrm{H}), 3.28(\mathrm{~s}, 3 \mathrm{H})$, $2.77(\mathrm{~d}, J=13.3 \mathrm{~Hz}, 1 \mathrm{H}), 2.39(\mathrm{~d}, J=13.3 \mathrm{~Hz}, 1 \mathrm{H}), 1.93(\mathrm{~m}, 2 \mathrm{H}), 1.86(\mathrm{~s}, 3 \mathrm{H}), 1.86(\mathrm{~m}, 1 \mathrm{H}), 1.80(\mathrm{~m}, 1 \mathrm{H})$, 1.47 (m, 3H), $1.22(\mathrm{~m}, 1 \mathrm{H}) ;{ }^{13} \mathrm{C}$ NMR $\left(125 \mathrm{MHz}, \mathrm{CDCl}_{3}\right) \square 142.1,136.6,130.7,129.1,126.2,115.8,78.6,53.8$, 48.9, 41.6, 31.9, 31.9, 30.1, 25.3, 21.9; IR (thin film) 3074, 2928, 2855, $1444 \mathrm{~cm}^{-1}$; HRMS (CI) m/z calcd for $\mathrm{C}_{17} \mathrm{H}_{24} \mathrm{OS}[\mathrm{M}]^{+}$276.1548, found 276.1549. Anal. Calcd for $\mathrm{C}_{17} \mathrm{H}_{24} \mathrm{OS}$ : C, 73.86; H, 8.75. Found: C, 73.89; H, 8.73 .

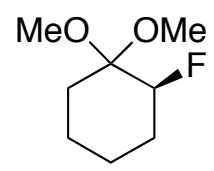

$11 \mathrm{a}$

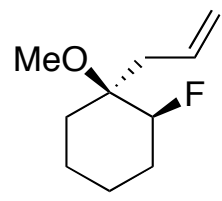

$12 a$

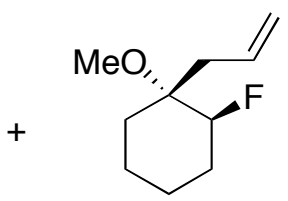

$13 a$

Fluorocyclohexanes 12a and 13a. The standard allylation procedure was followed with fluoro acetal $11 \mathrm{a}(0.150 \mathrm{~g}, 0.93 \mathrm{mmol})$, allyltrimethylsilane $(0.60 \mathrm{~mL}, 3.70 \mathrm{mmol})$, and TMSOTf $(0.20 \mathrm{~mL}, 1.11 \mathrm{mmol})$. GC and ${ }^{1} \mathrm{H}$ NMR spectroscopic analysis of the unpurified product indicated a pair of diastereomers in a 40:60 ratio (0.066 g, 42\%): ${ }^{1} \mathrm{H} \mathrm{NMR}\left(400 \mathrm{MHz}, \mathrm{CDCl}_{3}\right) \square 5.84(\mathrm{~m}, 1 \mathrm{H}), 5.14(\mathrm{~m}, 3 \mathrm{H}), 4.55(\mathrm{~m}, 0.3 \mathrm{H}), 4.47(\mathrm{dd}, J=3.8$, $10.4 \mathrm{~Hz}, 0.5 \mathrm{H}), 4.43(\mathrm{~m}, 0.3 \mathrm{H}), 4.35(\mathrm{dd}, J=3.8,10.4 \mathrm{~Hz}, 0.5 \mathrm{H}), 3.35(\mathrm{~s}, 3 \mathrm{H}), 3.32(\mathrm{~s}, 1.8 \mathrm{H}), 1.78(\mathrm{~m}, 5 \mathrm{H})$, $1.68(\mathrm{~m}, 6 \mathrm{H}), 2.45(\mathrm{~m}, 4 \mathrm{H}), 1.44(\mathrm{~m}, 5 \mathrm{H}), 1.27(\mathrm{~m}, 3 \mathrm{H})$. 
Purification of the resultant residue by silica gel chromatography (0:100 to 2:98 $\mathrm{Et}_{2} \mathrm{O}$ :pentane) resulted in decomposition. Mass spectrometry was obtained for unpurified major isomer (27a) and minor isomer (28a) as a mixture of diastereomers. HRMS (ESI) $m / z$ calcd for $\mathrm{C}_{10} \mathrm{H}_{17} \mathrm{FNaO}[\mathrm{M}+\mathrm{Na}]^{+}$172.1264, found 172.1262.

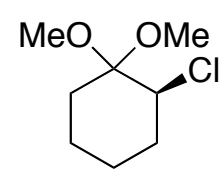

11b

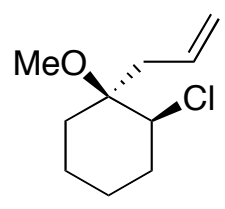

12b

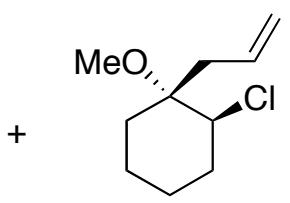

$13 b$

Chlorocyclohexanes 12b and 13b. The standard allylation procedure was followed with chloro acetal $11 \mathrm{~b}(0.212 \mathrm{~g}, 1.20 \mathrm{mmol})$, allyltrimethylsilane $(0.754 \mathrm{~mL}, 4.70 \mathrm{mmol})$, and $\mathrm{BF}_{3} \cdot \mathrm{OEt}_{2}(0.179 \mathrm{~mL}, 1.42 \mathrm{mmol})$. GC and ${ }^{1} \mathrm{H}$ NMR spectroscopic analysis of the unpurified product indicated a pair of diastereomers in a 17:83 ratio. Purification of the resultant residue by silica gel chromatography (0:100 to 2:98 EtOAc:hexanes) yielded the product as a clear oil $(0.160 \mathrm{~g}, 73 \%)$. The major isomer 12b was isolated as a pure sample while the minor isomer $\mathbf{1 3 b}$ was isolated as a mixture of $\mathbf{1 2 b}$ and $\mathbf{1 3 b}$. IR, mass spectrometry, and combustion analysis data was obtained for major isomer (12b) and minor isomer (13b) as a mixture of diastereomers. IR (thin film) 2956, 1075, $742 \mathrm{~cm}^{-1}$; HRMS (ESI) $\mathrm{m} / z$ calcd for $\mathrm{C}_{10} \mathrm{H}_{17} \mathrm{ClNaO}[\mathrm{M}+\mathrm{Na}]^{+} 211.0866$, found 211.0871. Anal. Calcd for $\mathrm{C}_{10} \mathrm{H}_{17} \mathrm{ClO}$ : C, 63.65; H, 9.08. Found: $\mathrm{C}, 63.35 ; \mathrm{H}, 9.13$.

Major Isomer (12b). ${ }^{1} \mathrm{H}$ NMR $\left(500 \mathrm{MHz}, \mathrm{CDCl}_{3}\right) \square 5.80$ (ddt, $\left.J=17.2,10.4,7.5 \mathrm{~Hz}, 1 \mathrm{H}\right), 5.19(\mathrm{~d}, J=17.0 \mathrm{~Hz}$, $1 \mathrm{H}), 5.14(\mathrm{~d}, J=10.1 \mathrm{~Hz}, 1 \mathrm{H}), 3.94(\mathrm{dd}, J=10.7,3.8 \mathrm{~Hz}, 1 \mathrm{H}), 3.28(\mathrm{~s}, 3 \mathrm{H}), 2.57(\mathrm{dd}, J=13.4,7.7 \mathrm{~Hz}, 1 \mathrm{H})$, $2.37(\mathrm{dd}, J=13.3,7.5 \mathrm{~Hz}, 1 \mathrm{H}), 2.11(\mathrm{dq}, J=4.2,12.1 \mathrm{~Hz}, 1 \mathrm{H}), 1.91(\mathrm{~m}, 2 \mathrm{H}) 1.41(\mathrm{~m}, 5 \mathrm{H}) ;{ }^{13} \mathrm{C}$ NMR $(125$ $\left.\mathrm{MHz}, \mathrm{CDCl}_{3}\right) \square 133.4,119.2,77.1,65.5,48.9,39.2,32.2,30.6,25.5,20.9$.

Minor Isomer (13b). ${ }^{1} \mathrm{H}$ NMR $\left(500 \mathrm{MHz}, \mathrm{CDCl}_{3}\right.$, distinctive peaks) $\square 4.08(\mathrm{~m}, 1 \mathrm{H}), 3.25(\mathrm{~s}, 3 \mathrm{H}), 2.44(\mathrm{dd}, J=$ 15.0, $7.5 \mathrm{~Hz}, 1 \mathrm{H}), 2.19(\mathrm{~m}, 1 \mathrm{H}) ;{ }^{13} \mathrm{C} \mathrm{NMR}\left(125 \mathrm{MHz}, \mathrm{CDCl}_{3}\right.$, distinctive peaks) 132.6, 118.6, 62.1, 49.1, 37.4, 20.9 .

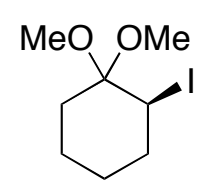

$11 \mathrm{c}$

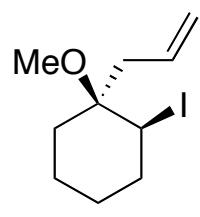

12c

Iodocyclohexane 12c. The standard allylation procedure was followed with iodo acetal 11c $(0.168 \mathrm{~g}$,

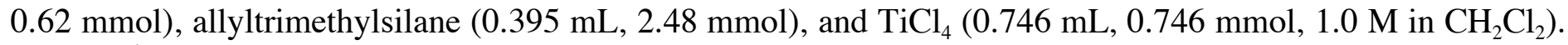
GC and ${ }^{1} \mathrm{H}$ NMR spectroscopic analysis of the unpurified product detected only a single isomer was present. Purification of the resultant residue by silica gel chromatography (2:98 EtOAc: hexanes) yielded the product as a colorless oil $(0.137 \mathrm{~g}, 79 \%) ;{ }^{1} \mathrm{H}$ NMR $\left(500 \mathrm{MHz}, \mathrm{CDCl}_{3}\right) \square 5.72(\mathrm{ddt}, J=17.0,12.4,5.0 \mathrm{~Hz}, 1 \mathrm{H}), 5.22(\mathrm{~d}, J=$ $16.9 \mathrm{~Hz}, 1 \mathrm{H}), 5.14(\mathrm{~d}, J=10.1 \mathrm{~Hz}, 1 \mathrm{H}), 4.23(\mathrm{dd}, J=10.2,4.2 \mathrm{~Hz}, 1 \mathrm{H}), 3.24(\mathrm{~s}, 3 \mathrm{H}), 2.39(\mathrm{~m}, 3 \mathrm{H}), 2.13(\mathrm{~m}$, $1 \mathrm{H}), 1.97(\mathrm{~m}, 1 \mathrm{H}), 1.52(\mathrm{~m}, 4 \mathrm{H}), 1.31(\mathrm{~m}, 1 \mathrm{H}) ;{ }^{13} \mathrm{C} \mathrm{NMR}\left(125 \mathrm{MHz}, \mathrm{CDCl}_{3}\right) \square 133.2,119.4,76.2,49.0,43.8$, 36.1, 29.7, 21.5; IR (thin film) 3076, 1440, 2935, $669 \mathrm{~cm}^{-1}$; HRMS (CI) $\mathrm{m} / z$ calcd for $\mathrm{C}_{10} \mathrm{H}_{17} \mathrm{INaO}[\mathrm{M}+\mathrm{Na}]^{+}$ 303.0222, found 303.0127. Anal. Calcd for $\mathrm{C}_{10} \mathrm{H}_{17} \mathrm{OI}$ : C, 42.87; H, 6.12. Found: C, 43.12; H, 5.95. 


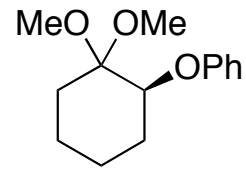

$11 d$

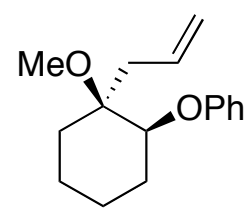

12d

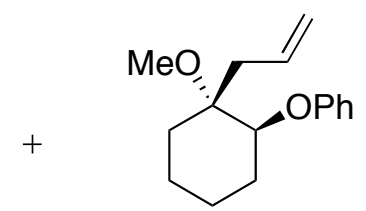

13d

Phenoxycyclohexanes 12d and 13d. The standard allylation procedure was followed with acetal 11d $(0.190 \mathrm{~g}, 0.81 \mathrm{mmol})$, allyltrimethylsilane $(0.511 \mathrm{~mL}, 3.20 \mathrm{mmol})$, and $\mathrm{BF}_{3} \cdot \mathrm{OEt}_{2}(0.121 \mathrm{~mL}, 0.97 \mathrm{mmol})$. GC and ${ }^{1} \mathrm{H}$ NMR spectroscopic analysis of the unpurified product indicated a pair of diastereomers in a 34:66 ratio. Separation of the diastereomers was achieved by purification of the resultant residue by silica gel chromatography (3:97 EtOAc:hexanes) to yield the product as a colorless oil $(0.171 \mathrm{~g}, 87 \%)$. Mass spectrometry data was obtained for major isomer (12d) and minor isomer (13d) as a mixture of diastereomers: HRMS (ESI) $m / z$ calcd for $\mathrm{C}_{16} \mathrm{H}_{22} \mathrm{NaO}_{2}[\mathrm{M}+\mathrm{Na}]^{+} 269.1518$, found 269.1513.

Major Isomer (12d). ${ }^{1} \mathrm{H} \mathrm{NMR}\left(500 \mathrm{MHz}, \mathrm{CDCl}_{3}\right) \square 7.13(\mathrm{~m}, 2 \mathrm{H}), 6.83(\mathrm{~m}, 3 \mathrm{H}), 5.78$ (ddt, J = 17.2, $12.2,5.2$ $\mathrm{Hz}, 1 \mathrm{H}), 4.99(\mathrm{~d}, J=10.1 \mathrm{~Hz}, 1 \mathrm{H}), 4.98(\mathrm{~d}, J=17.1 \mathrm{~Hz}, 1 \mathrm{H}), 4.08(\mathrm{dd}, J=10.5,3.7 \mathrm{~Hz}, 1 \mathrm{H}), 3.37(\mathrm{~s}, 3 \mathrm{H}), 2.54$ $(\mathrm{dd}, J=13.6,7.5 \mathrm{~Hz}, 1 \mathrm{H}), 2.47(\mathrm{dd}, J=13.6,7.2 \mathrm{~Hz}, 1 \mathrm{H}), 1.94(\mathrm{~m}, 1 \mathrm{H}), 1.76(\mathrm{~m}, 2 \mathrm{H}), 1.58(\mathrm{~m}, 2 \mathrm{H}), 1.24(\mathrm{~m}$, $1 \mathrm{H}), 1.13(\mathrm{ddd}, J=13.9,12.1,4.1 \mathrm{~Hz}, 1 \mathrm{H}), 1.04(\mathrm{~m}, 1 \mathrm{H}) ;{ }^{13} \mathrm{C} \mathrm{NMR}\left(125 \mathrm{MHz}, \mathrm{CDCl}_{3}\right) \square 158.5,133.9,129.7$, 120.9, 118.5, 116.1, 80.3, 77.6 49.8, 38.2, 31.7, 26.1, 24.2, 21.0; IR (thin film) 2929, 2860, $1597,1493 \mathrm{~cm}^{-1}$. Anal. Calcd for $\mathrm{C}_{16} \mathrm{H}_{22} \mathrm{O}_{2}$ : C, 78.01; H, 9.00. Found: C, 78.18; H, 9.07.

Minor Isomer (13d). ${ }^{1} \mathrm{H}$ NMR $\left(500 \mathrm{MHz} \mathrm{CDCl}_{3}\right) \square 7.24(\mathrm{~m}, 2 \mathrm{H}), 6.90(\mathrm{~m}, 3 \mathrm{H}), 5.78$ (ddt, $J=21.1,12.8,8.6$ $\mathrm{Hz}, 1 \mathrm{H}), 5.03(\mathrm{~d}, J=12.8 \mathrm{~Hz}, 1 \mathrm{H}), 4.95(\mathrm{~d}, J=21.2 \mathrm{~Hz}, 1 \mathrm{H}), 4.26(\mathrm{t}, J=3.6 \mathrm{~Hz}, 1 \mathrm{H}), 3.25(\mathrm{~s}, 3 \mathrm{H}) 2.44(\mathrm{dd}, J=$ 18.5, $9.6 \mathrm{~Hz}, 1 \mathrm{H}), 2.36(\mathrm{dd}, J=18.1,8.5 \mathrm{~Hz}, 1 \mathrm{H}) 1.78(\mathrm{~m}, 4 \mathrm{H}), 1.48(\mathrm{~m}, 3 \mathrm{H}), 1.36(\mathrm{~m}, 1 \mathrm{H}) ;{ }^{13} \mathrm{C}$ NMR $(125$ $\left.\mathrm{MHz}, \mathrm{CDCl}_{3}\right) \square 157.9,133.4,129.7,120.9,118.3,116.2,77.6,75.1,48.6,36.8,28.9,24.6,21.1,20.2$; IR (thin film) 2933, 2826, 1598, $1495 \mathrm{~cm}^{-1}$. Anal. Calcd for $\mathrm{C}_{16} \mathrm{H}_{22} \mathrm{O}_{2}$ : C, 78.01; H, 9.00. Found: C, 78.16; H, 9.19.

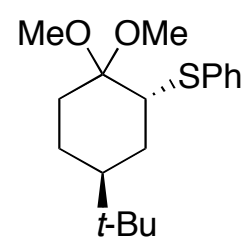

19

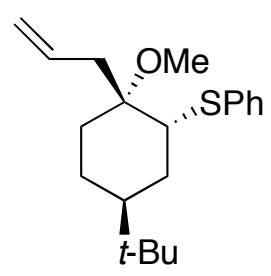

20

Allyl Product 20. The standard allylation procedure was followed with acetal 19 (0.070 g, $0.23 \mathrm{mmol})$, allyltrimethylsilane $(0.144 \mathrm{~mL}, 0.91 \mathrm{mmol})$, and $\mathrm{MeAlCl}_{2}(0.27 \mathrm{~mL}, 0.27 \mathrm{mmol}, 1.0 \mathrm{M}$ in hexane $)$. GC and ${ }^{1} \mathrm{H}$ NMR spectroscopic analysis of the unpurified product detected only a single isomer was present. Purification of the resultant residue by silica gel chromatography (0:100 to 2:98 EtOAc:hexanes) yielded the product as a pale yellow oil $(0.062 \mathrm{~g}, 87 \%)$ : ${ }^{1} \mathrm{H}$ NMR $\left(500 \mathrm{MHz}, \mathrm{C}_{6} \mathrm{D}_{6}\right) \square 7.48(\mathrm{~m}, 2 \mathrm{H}), 7.05(\mathrm{t}, J=7.5 \mathrm{~Hz}, 2 \mathrm{H}), 6.97$ (m, $1 \mathrm{H}), 5.92(\mathrm{ddt}, J=17.2,10.2,6.97 \mathrm{~Hz}, 1 \mathrm{H}), 5.10(\mathrm{~m}, 2 \mathrm{H}), 3.58(\mathrm{~m}, 1 \mathrm{H}), 3.33(\mathrm{~s}, 3 \mathrm{H}), 2.31(\mathrm{dd}, J=15.0,7.5$ $\mathrm{Hz}, 1 \mathrm{H}), 2.22(\mathrm{dd}, J=15.0,7.1 \mathrm{~Hz}, 1 \mathrm{H}), 2.00(\mathrm{td}, J=13.3,3.8 \mathrm{~Hz}, 1 \mathrm{H}), 1.82(\mathrm{tt}, J=12.5,3.7 \mathrm{~Hz}, 1 \mathrm{H}), 1.76$ $(\mathrm{dq}, J=13.1,1.8 \mathrm{~Hz}, 1 \mathrm{H}), 1.67(\mathrm{ddt}, J=15.2,3.1 \mathrm{~Hz}, 1 \mathrm{H}), 1.56(\mathrm{~m}, 1 \mathrm{H}), 1.15(\mathrm{ddd}, J=14.3,12.4,3.4 \mathrm{~Hz}, 1 \mathrm{H})$, $1.01(\mathrm{dq}, J=3.8,13.4 \mathrm{~Hz}, 1 \mathrm{H}), 0.77(\mathrm{~s}, 9 \mathrm{H}) ;{ }^{13} \mathrm{C} \mathrm{NMR}\left(125 \mathrm{MHz}, \mathrm{CDCl}_{3}\right) \square 135.9,133.4,133.4,129.1,127.2$, 118.1, 53.9, 48.8, 40.5, 36.3, 32.2, 30.8, 28.1, 27.8, 27.5, 23.7; IR (thin film) 2948, 2869, 1480, $1077 \mathrm{~cm}^{-1}$; HRMS (ESI) $m / z$ calcd for $\mathrm{C}_{20} \mathrm{H}_{30} \mathrm{ONaS}[\mathrm{M}+\mathrm{Na}]^{+} 341.1915$, found 341.1921. Anal Calcd for for $\mathrm{C}_{20} \mathrm{H}_{30} \mathrm{OS}: \mathrm{C}$, 75.42; H, 9.49. Found: C, 75.02; H, 9.67. 
<smiles></smiles>

19

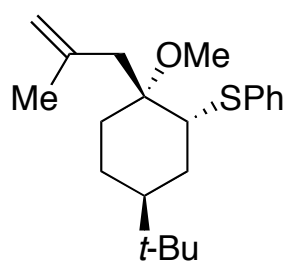

21

Methallyl Product 21. The standard allylation procedure was followed with acetal $19(0.072 \mathrm{~g}, 0.23$ mmol), 2-methylpropenyltrimethylsilane $(0.160 \mathrm{~mL}, 0.93 \mathrm{mmol})$, and $\mathrm{TiCl}_{4}(0.28 \mathrm{~mL}, 0.28 \mathrm{mmol}, 1.0 \mathrm{M}$ in $\mathrm{CH}_{2} \mathrm{Cl}_{2}$ ). GC and ${ }^{1} \mathrm{H}$ NMR spectroscopic analysis of the unpurified product detected only a single isomer was present. Purification of the resultant residue by silica gel chromatography (0:100 to 2:98 EtOAc:hexanes) yielded the product as a pale yellow oil $(0.066 \mathrm{~g}, 86 \%):{ }^{1} \mathrm{H} \mathrm{NMR}\left(500 \mathrm{MHz}, \mathrm{C}_{6} \mathrm{D}_{6}\right) \square 7.49(\mathrm{~m}, 2 \mathrm{H}), 7.05(\mathrm{~m}$, 2H), $6.97(\mathrm{tt}, J=2.0,1.2 \mathrm{~Hz}, 1 \mathrm{H}), 4.95(\mathrm{~s}, 1 \mathrm{H}), 4.87(\mathrm{~s}, 1 \mathrm{H}), 3.60(\mathrm{~m}, 1 \mathrm{H}), 3.23(\mathrm{~s}, 3 \mathrm{H}), 2.28(\mathrm{ddd}, J=14.0$, 11.7, Hz, 2H), 2.01 (td, $J=13.5,4.3 \mathrm{~Hz}, 1 \mathrm{H}), 1.89(\mathrm{~s}, 3 \mathrm{H}), 1.87(\mathrm{dt}, J=12.6,3.6 \mathrm{~Hz}, 1 \mathrm{H}), 1.80(\mathrm{dq}, J=13.0$, $2.8 \mathrm{~Hz}, 1 \mathrm{H}$ ), 1.75 (ddt, $J=15.0,3.5 \mathrm{~Hz}, 1 \mathrm{H}), 1.59$ (ddt, $J=13.4,6.5,3.4 \mathrm{~Hz}, 1 \mathrm{H}), 1.24$ (ddd, $J=14.4,12.5,3.3$ $\mathrm{Hz}, 1 \mathrm{H}), 1.08(\mathrm{dq}, J=3.8,13.2 \mathrm{~Hz}, 1 \mathrm{H}) 0.79(\mathrm{~s}, 9 \mathrm{H}) ;{ }^{13} \mathrm{C}$ NMR $\left(125 \mathrm{MHz}, \mathrm{CDCl}_{3}\right) \square 142.4,136.3,133.4,129.2$, $127.3,115.0,78.5,54.3,48.9,40.5,38.3,32.4,32.2,28.5,27.9,24.2,24.1$; IR (thin film) $3059,2949,1468 \mathrm{~cm}^{-}$ ${ }^{1}$; HRMS (EI) $m / z$ calcd for $\mathrm{C}_{21} \mathrm{H}_{32} \mathrm{OS}[\mathrm{M}]^{+} 332.2174$, found 332.2180.<smiles>COC1(OC)CCC(C)(C)CC1Sc1ccccc1</smiles>

30

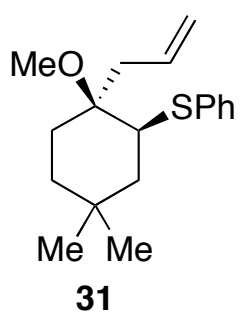

31

Allyl Product 31. The standard allylation procedure was followed with acetal $\mathbf{3 0}(0.071 \mathrm{~g}, 0.25 \mathrm{mmol})$, allyltrimethylsilane $(0.161 \mathrm{~mL}, 1.01 \mathrm{mmol})$, and $\mathrm{BF}_{3} \cdot \mathrm{OEt}_{2}(0.038 \mathrm{~mL}, 0.30 \mathrm{mmol})$. GC and ${ }^{1} \mathrm{H} \mathrm{NMR}$ spectroscopic analysis of the unpurified product detected only a single isomer was present. Purification of the resultant residue by silica gel chromatography (0:100 to 2:98 EtOAc:hexanes) yielded the product as a colorless oil $(0.067 \mathrm{~g}, 92 \%):{ }^{1} \mathrm{H}$ NMR $\left(400 \mathrm{MHz}, \mathrm{CDCl}_{3}\right) \square 7.40(\mathrm{~m}, 2 \mathrm{H}), 7.28(\mathrm{~m}, 2 \mathrm{H}), 7.15(\mathrm{~m}, 1 \mathrm{H}), 5.82(\mathrm{ddt}, J=$ $17.2,12.4,7.5 \mathrm{~Hz}, 1 \mathrm{H}), 5.18(\mathrm{~d}, J=17.0 \mathrm{~Hz}, 1 \mathrm{H}) 5.11(\mathrm{~d}, J=12.2 \mathrm{~Hz}, 1 \mathrm{H}), 3.27(\mathrm{~m}, 1 \mathrm{H}), 3.26(\mathrm{~s}, 3 \mathrm{H}), 2.80$ $(\mathrm{dd}, J=12.9,7.7 \mathrm{~Hz}, 1 \mathrm{H}), 2.41(\mathrm{dd}, J=12.9,7.4 \mathrm{~Hz}, 1 \mathrm{H}), 1.89(\mathrm{t}, J=12.9 \mathrm{~Hz}, 1 \mathrm{H}), 1.80(\mathrm{dt}, J=15.0,3.3 \mathrm{~Hz}$, $1 \mathrm{H}), 1.60(\mathrm{td}, J=14.5,3.9 \mathrm{~Hz}, 1 \mathrm{H}), 1.49(\mathrm{ddd}, J=13.3,4.1,2.5 \mathrm{~Hz}, 1 \mathrm{H}), 1.42(\mathrm{td}, J=13.2,3.7 \mathrm{~Hz}, 1 \mathrm{H}), 1.12$ (ddd, $J=12.9,6.0,2.9 \mathrm{~Hz}, 1 \mathrm{H}), 0.89$ (s, 3H), $0.84(\mathrm{~s}, 3 \mathrm{H}) ;{ }^{13} \mathrm{C}$ NMR $\left(125 \mathrm{MHz}, \mathrm{CDCl}_{3}\right) \square 137.1,134.1,130.4$, 129.1, 126.2, 119.2, 76.8, 50.8, 48.9, 43.1, 40.4, 33.9, 32.6, 31.8, 26.7, 24.1; IR (thin film) 3074, 2951, 1481 $\mathrm{cm}^{-1}$; HRMS (CI) $\mathrm{m} / z$ calcd for $\mathrm{C}_{18} \mathrm{H}_{26} \mathrm{NaOS}[\mathrm{M}+\mathrm{Na}]^{+}$313.1602, found 313.1608. Anal. Calcd for $\mathrm{C}_{18} \mathrm{H}_{26} \mathrm{OS}$ : C, 74.43; H, 9.02. Found: C, 74.69; H, 9.26.

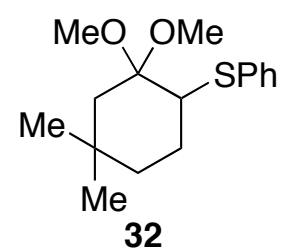

32

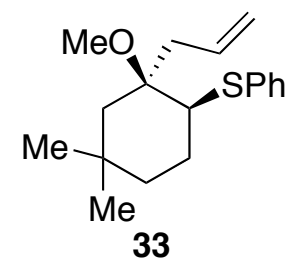

33 
Allyl Product 33. The standard allylation procedure was followed with acetal $32(0.091 \mathrm{~g}, 0.33 \mathrm{mmol})$, allyltrimethylsilane $(0.206 \mathrm{~mL}, 1.33 \mathrm{mmol})$, and $\mathrm{BF}_{3} \cdot \mathrm{OEt}_{2}(0.050 \mathrm{~mL}, 0.39 \mathrm{mmol})$. GC and ${ }^{1} \mathrm{H} \mathrm{NMR}$ spectroscopic analysis of the unpurified product detected only a single isomer was present. Purification of the resultant residue by silica gel chromatography (0:100 to 2:98 EtOAc:hexanes) yielded the product as a pale yellow oil (0.084 g, 89\%): ${ }^{1} \mathrm{H}$ NMR (500 MHz, $\left.\mathrm{CDCl}_{3}\right) \square 7.40$ (m, 2H), 7.28 (m, 2H), 7.19 (m, $\left.1 \mathrm{H}\right), 5.81$ (ddt, $J$ $=17,12,4.8 \mathrm{~Hz}, 1 \mathrm{H}), 5.19(\mathrm{~m}, 1 \mathrm{H}), 5.12(\mathrm{~m}, 1 \mathrm{H}), 3.24(\mathrm{~s}, 3 \mathrm{H}), 2.98(\mathrm{dd}, J=12.2,4.2 \mathrm{~Hz}, 1 \mathrm{H}), 2.79(\mathrm{dd}, J=$ 13.0, 7.6 Hz, 1H), $2.34(\mathrm{dd}, J=13.0,7.4 \mathrm{~Hz}, 1 \mathrm{H}), 2.10(\mathrm{~m}, 1 \mathrm{H}), 1.78(\mathrm{dd}, J=15.1,2.7 \mathrm{~Hz}, 1 \mathrm{H}), 1.73(\mathrm{~m}, 1 \mathrm{H})$, $1.41(\mathrm{~m}, 1 \mathrm{H}), 1.20(\mathrm{~d}, J=15.1 \mathrm{~Hz}, 1 \mathrm{H}), 1.10(\mathrm{~m}, 1 \mathrm{H}), 1.10(\mathrm{~s}, 3 \mathrm{H}), 0.88(\mathrm{~s}, 3 \mathrm{H}) ;{ }^{13} \mathrm{C} \mathrm{NMR}\left(125 \mathrm{MHz}, \mathrm{CDCl}_{3}\right) \square$ 136.8, 134.4, 131.4, 129.1, 126.5, 119.2, 78.8, 54.9, 49.2, 41.7, 40.9, 40.0, 34.1, 30.9, 26.9, 25.8; IR (thin film) 3075, 2951, 2868, $1438 \mathrm{~cm}^{-1}$; HRMS (CI) $\mathrm{m} / z$ calcd for $\mathrm{C}_{18} \mathrm{H}_{26} \mathrm{NaOS}[\mathrm{M}+\mathrm{Na}]^{+}$313.1602, found: 313.1613 . Anal. Calcd for $\mathrm{C}_{18} \mathrm{H}_{26} \mathrm{OS}$ : C, 74.43; H, 9.02. Found: C, 74.32; H, 9.06.

General Procedure for Cyanation of 2-Phenylsulfanylcyclohexanone Dimethyl Acetals: A solution of the acetal in $\mathrm{CH}_{2} \mathrm{Cl}_{2}(0.10 \mathrm{M})$ was treated with $\mathrm{Me}_{3} \mathrm{SiCN}$ (4.0 equiv) and then cooled to $-78{ }^{\circ} \mathrm{C}$. The appropriate Lewis acid (1.2 equiv) was added dropwise and the reaction mixture was allowed to warm to $22^{\circ} \mathrm{C}$. After $18 \mathrm{~h}$, the reaction mixture was cooled to $-78{ }^{\circ} \mathrm{C}$ and treated with a 1:1:1 solution of $\mathrm{Et}_{3} \mathrm{~N}, \mathrm{MeOH}$ and $\mathrm{CH}_{2} \mathrm{Cl}_{2}$ (1 $\mathrm{mL}$ per $\mathrm{mL}$ of reaction volume). The reaction mixture was poured into a separatory funnel containing saturated aqueous $\mathrm{NaHCO}_{3}(1 \mathrm{~mL}$ per $\mathrm{mL}$ of reaction volume $)$ and extracted with 3 portions of $\mathrm{CH}_{2} \mathrm{Cl}_{2}$ (1 mL per $\mathrm{mL}$ of acetal). The organic layers were washed with a saturated sodium chloride solution, dried $\left(\mathrm{Na}_{2} \mathrm{SO}_{4}\right)$, and concentrated in vacuo. The unpurified mixture was analyzed by GC and ${ }^{1} \mathrm{H} \mathrm{NMR}$ spectroscopy, then purified as indicated.

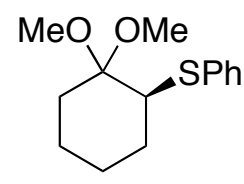

7

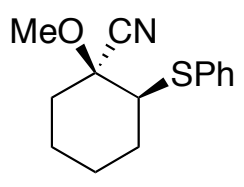

10

Nitrile 10. The cyanation procedure was followed with acetal 7 (0.094 g, $0.37 \mathrm{mmol}), \mathrm{Me}_{3} \mathrm{SiCN}(0.198$ $\mathrm{mL}, 1.50 \mathrm{mmol})$, and $\mathrm{BF}_{3} \cdot \mathrm{OEt}_{2}(0.056 \mathrm{~mL}, 0.45 \mathrm{mmol})$. $\mathrm{GC}$ and ${ }^{1} \mathrm{H} \mathrm{NMR}$ spectroscopic analysis of the unpurified product detected only a single isomer was present. Purification of the resultant residue by silica gel chromatography (0:100 to 5:95 EtOAc:hexanes) yielded the product as a white solid (0.086 g, 93\%). X-ray quality crystals were grown from a $3: 1$ mixture of $\mathrm{CHCl}_{3}$ and hexanes in which slow evaporation provided the crystal: $\mathrm{mp} 80-83{ }^{\circ} \mathrm{C} ;{ }^{1} \mathrm{H}$ NMR $\left(500 \mathrm{MHz}, \mathrm{CDCl}_{3}\right) \square 7.29-7.53(\mathrm{~m}, 5 \mathrm{H}), 3.46(\mathrm{~s}, 3 \mathrm{H}), 3.34(\mathrm{~m}, 1 \mathrm{H}), 2.32(\mathrm{~m}$, $1 \mathrm{H}), 1.94(\mathrm{~m}, 2 \mathrm{H}), 1.73(\mathrm{~m}, 2 \mathrm{H}), 1.57(\mathrm{~m}, 2 \mathrm{H}), 1.35(\mathrm{~m}, 1 \mathrm{H}) ;{ }^{13} \mathrm{C} \mathrm{NMR}\left(125 \mathrm{MHz}, \mathrm{CDCl}_{3}\right) \square$ 135.0, 133.3, 129.4, 128.0, 119.4, 78.2, 55.2, 53.1, 32.7, 29.6, 23.8, 20.5; IR (thin film) 2943, 2082, 2866, 2260, $1444 \mathrm{~cm}^{-1}$; HRMS (CI) $\mathrm{m} / \mathrm{z}$ calcd for $\mathrm{C}_{14} \mathrm{H}_{18} \mathrm{NOS}[\mathrm{M}+\mathrm{H}]^{+} 248.1071$, found 248.1073. Anal. Calcd for $\mathrm{C}_{14} \mathrm{H}_{17} \mathrm{NOS}$ : C, 67.98; H, 6.93; N, 5.66. Found: C, 67.97; H, 7.00; N, 5.63.<smiles></smiles>

19

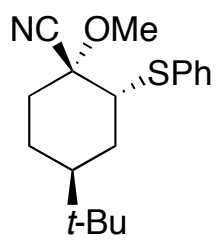

22 
Nitrile 22. The standard cyanation procedure was followed with acetal $19(0.366 \mathrm{~g}, 1.41 \mathrm{mmol})$, $\mathrm{Me}_{3} \mathrm{SiCN}(0.750 \mathrm{~mL}, 5.63 \mathrm{mmol})$, and $\mathrm{BF}_{3} \cdot \mathrm{OEt}_{2}(0.213 \mathrm{~mL}, 1.68 \mathrm{mmol})$. GC and ${ }^{1} \mathrm{H}$ NMR spectroscopic analysis of the unpurified product detected only a single isomer was present. Purification of the resultant residue by silica gel chromatography (0:100 to 2:98 EtOAc:hexanes) yielded the product as a white solid $(0.321$ g, 89\%): ${ }^{1} \mathrm{H}$ NMR $\left(500 \mathrm{MHz}, \mathrm{CDCl}_{3}\right) \square 7.49(\mathrm{~m}, 2 \mathrm{H}), 7.30(\mathrm{~m}, 3 \mathrm{H}), 3.87(\mathrm{~m}, 1 \mathrm{H}), 3.45(\mathrm{~s}, 3 \mathrm{H}), 2.07(\mathrm{~m}, 1 \mathrm{H})$, $2.03(\mathrm{dd}, J=13.2,3.9 \mathrm{~Hz}, 1 \mathrm{H}), 1.99(\mathrm{~m}, 1 \mathrm{H}), 1.89(\mathrm{~m}, 1 \mathrm{H}), 1.70(\mathrm{ddd}, J=14.2,12.3,3.3 \mathrm{~Hz}, 1 \mathrm{H}), 1.60(\mathrm{~m}$, $1 \mathrm{H}), 1.40$ (ddd, $J=25.8,13.3,4.2 \mathrm{~Hz}, 1 \mathrm{H}) 0.83(\mathrm{~s}, 9 \mathrm{H}) ;{ }^{13} \mathrm{C}$ NMR $\left(125 \mathrm{MHz}, \mathrm{CDCl}_{3}\right) \square 134.2,133.7,129.4$, 128.2, 119.1, 78.9, 53.0, 52.6, 40.8, 32.2, 31.7, 30.2, 27.7, 24.4; IR (thin film) 3060, 2960, 2870, $2260 \mathrm{~cm}^{-1}$; HRMS (EI) $\mathrm{m} / z$ calcd for $\mathrm{C}_{18} \mathrm{H}_{25} \mathrm{NOS}[\mathrm{M}]^{+} 303.1657$, found: 303.1659. Anal. Calcd for $\mathrm{C}_{18} \mathrm{H}_{25} \mathrm{NOS}$ : C, 71.24; H, 8.30; N, 4.62. Found: C, 71.51; H, 8.46; N, 4.68.

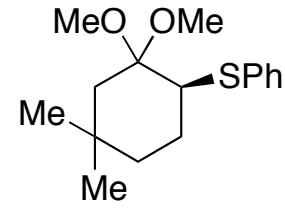

32

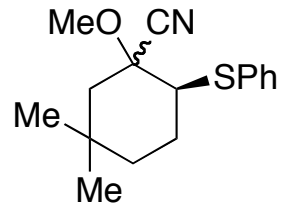

34

Nitrile 34. The standard cyanation procedure was followed with acetal $32(0.222 \mathrm{~g}, 0.79 \mathrm{mmol})$, $\mathrm{Me}_{3} \mathrm{SiCN}(0.423 \mathrm{~mL}, 3.17 \mathrm{mmol})$, and $\mathrm{BF}_{3}{ }^{\cdot} \mathrm{OEt}_{2}(0.120 \mathrm{~mL}, 0.95 \mathrm{mmol})$. $\mathrm{GC}$ and ${ }^{1} \mathrm{H}$ NMR spectroscopic analysis of the unpurified product indicated a pair of diastereomers in a ratio of 92:8. Purification of the resultant residue by silica gel chromatography (3:97 EtOAc:hexanes) yielded the product as a white solid $(0.198$ g, 91\%). The purified products were characterized as a mixture of diastereomers: ${ }^{1} \mathrm{H}$ NMR $\left(400 \mathrm{MHz}, \mathrm{CDCl}_{3}\right)$ $7.53(\mathrm{~m}, 2.3 \mathrm{H}), 7.31(\mathrm{~m}, 3.3 \mathrm{H}), 3.50(\mathrm{~s}, 3 \mathrm{H}), 3.47(\mathrm{~s}, 0.4 \mathrm{H}), 3.19(\mathrm{dd}, J=11.1,4.4 \mathrm{~Hz}, 1.1 \mathrm{H}), 2.23(\mathrm{dd}, J=$ $14.8,2.2 \mathrm{~Hz}, 1 \mathrm{H}), 2.01(\mathrm{~m}, 2.4 \mathrm{H}), 1.24(\mathrm{~m}, 1.7 \mathrm{H}), 1.09(\mathrm{~s}, 0.5 \mathrm{H}), 1.06(\mathrm{~s}, 3 \mathrm{H}), 1.01(\mathrm{~s}, 0.5 \mathrm{H}), 0.95(\mathrm{~s}, 3 \mathrm{H}) ;{ }^{13} \mathrm{C}$ NMR $\left(125 \mathrm{MHz}, \mathrm{CDCl}_{3}\right) \square 135.2,133.3,133.2,129.4,129.2,128.0,127.9,119.6,56.5,53.7,53.2,43.4,38.4$, 32.2, 31.4, 26.7, 26.5; IR (thin film) 3059, 2953, 2866, $2850 \mathrm{~cm}^{-1}$; HRMS (ESI) $\mathrm{m} / z$ calcd for $\mathrm{C}_{16} \mathrm{H}_{21} \mathrm{NNaOS}$ [M $+\mathrm{Na}^{+}$298.1241, found: 298.1248. Anal. Calcd for $\mathrm{C}_{16} \mathrm{H}_{21} \mathrm{NOS}: \mathrm{C}, 69.78 ; \mathrm{H}, 7.69 ; \mathrm{N}, 5.09$. Found: C, 69.92; $\mathrm{H}, 7.76 ; \mathrm{N}, 5.06$.

General Procedure for Grignard Addition to Cyclohexanones: To a cooled $\left(-78{ }^{\circ} \mathrm{C}\right)$ solution of cyclohexanone derivative (1.00 equiv) in THF $(0.10 \mathrm{M})$ was treated with Grignard nucleophile (2.40 equiv). The reaction mixture was warmed to $22{ }^{\circ} \mathrm{C}$ and stirred for $1.5 \mathrm{~h}$ before it was cooled to $0{ }^{\circ} \mathrm{C}$ and treated with $\mathrm{H}_{2} \mathrm{O}$ (1 mL per mmol cyclohexanone). The aqueous layer was extracted with three portions of $\mathrm{Et}_{2} \mathrm{O}(1 \mathrm{~mL}$ per $\mathrm{mmol}$ cyclohexanone). The combined organic layers were washed with a saturated sodium chloride solution ( $1 \mathrm{~mL}$ per $\mathrm{mL}$ of $\left.\mathrm{Et}_{2} \mathrm{O}\right)$, dried $\left(\mathrm{Na}_{2} \mathrm{SO}_{4}\right)$, and concentrated in vacuo. The unpurified mixture was analyzed by $\mathrm{GC}$ and ${ }^{1} \mathrm{H}$ NMR spectroscopy, then purified as indicated.

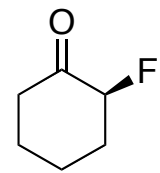

17

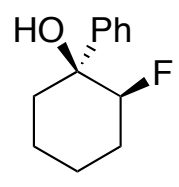

18

Fluorocyclohexanol 18. The standard Grignard addition procedure was followed with $\mathbf{1 7}$ ( $0.036 \mathrm{~g}, 0.31$ mmol) and phenylmagnesium bromide $\left(0.75 \mathrm{~mL}, 0.75 \mathrm{mmol}, 1.0 \mathrm{M}\right.$ in THF). GC and ${ }^{1} \mathrm{H}$ NMR spectroscopic analysis of the unpurified product indicated a single isomer was present. Purification of the resultant residue by 
silica gel chromatography (2:98 EtOAc:hexanes) yielded the product as a white solid $(0.057 \mathrm{~g}, 95 \%)$. X-ray quality crystals were grown from $\mathrm{CHCl}_{3}$ in which slow evaporation provided the crystal: $\mathrm{mp} 74{ }^{\circ} \mathrm{C} ;{ }^{1} \mathrm{H}$ NMR $\left(500 \mathrm{MHz}, \mathrm{CDCl}_{3}\right) \square 7.50(\mathrm{~d}, J=7.5 \mathrm{~Hz}, 2 \mathrm{H}), 7.37(\mathrm{t}, J=7.8 \mathrm{~Hz}, 2 \mathrm{H}), 7.28(\mathrm{~m}, 1 \mathrm{H}), 4.98(\mathrm{dt}, J=40.4,8.4 \mathrm{~Hz}$, $1 \mathrm{H}), 2.27(\mathrm{t}, J=2.3 \mathrm{~Hz}, 1 \mathrm{H}), 2.02(\mathrm{tdd}, J=9.0,8.9,3.8 \mathrm{~Hz}, 2 \mathrm{H}), 1.94(\mathrm{~m}, 1 \mathrm{H}), 1.83(\mathrm{~m}, 1 \mathrm{H}), 1.73(\mathrm{ddt}, J=$ $25.8,12.9,3.6 \mathrm{~Hz}, 1 \mathrm{H}), 1.60(\mathrm{~m}, 1 \mathrm{H}), 1.52(\mathrm{~m}, 1 \mathrm{H}), 1.39(\mathrm{~m}, 1 \mathrm{H}) ;{ }^{13} \mathrm{C} \mathrm{NMR}\left(125 \mathrm{MHz}, \mathrm{CDCl}_{3}\right) \square 145.7,128.7$, 127.5, 125.2, $95.2(\mathrm{~d}, J=176.3 \mathrm{~Hz}), 75.2(\mathrm{~d}, J=17.5 \mathrm{~Hz}), 39.3(\mathrm{~d}, J=15.0 \mathrm{~Hz}), 27.8(\mathrm{~d}, J=70.0 \mathrm{~Hz}), 23.9(\mathrm{~d}$, $J=45.0 \mathrm{~Hz}$ ), $21.1\left(\mathrm{~d}, J=5.0 \mathrm{~Hz}\right.$ ); IR (thin film) $3419,2924,1458 \mathrm{~cm}^{-1}$; HRMS (ESI) $\mathrm{m} / z$ calcd for $\mathrm{C}_{12} \mathrm{H}_{15} \mathrm{FNaO}$ $[\mathrm{M}+\mathrm{Na}]^{+}$217.1005, found 217.1004. Anal. Calcd for $\mathrm{C}_{12} \mathrm{H}_{15} \mathrm{FO}$ : C, 74.97; H, 8.23. Found: C, 74.90; H, 8.16.

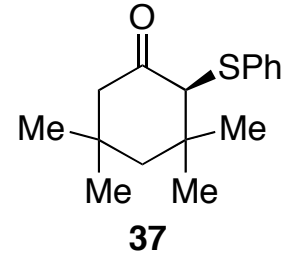

3,3,5,5-Tetramethylcyclohexanol 38. The standard Grignard addition procedure was followed with $\mathbf{3 7}$ $(0.044 \mathrm{~g}, 0.19 \mathrm{mmol})$ and phenylmagnesium bromide $\left(0.45 \mathrm{~mL}, 0.45 \mathrm{mmol}, 1.0 \mathrm{M}\right.$ in THF). GC and ${ }^{1} \mathrm{H}$ NMR spectroscopic analysis of the unpurified product detected only a single isomer was present. Purification of the resultant residue by silica gel chromatography (3:97 EtOAc:hexanes) yielded the product as a white solid (0.054 g, 84\%). X-ray quality crystals were grown from a 3:1 mixture of hexanes and $\mathrm{CH}_{2} \mathrm{Cl}_{2}$ in which slow evaporation provided the crystal: mp 108-110 ${ }^{\circ} \mathrm{C} ;{ }^{1} \mathrm{H}$ NMR $\left(500 \mathrm{MHz}, \mathrm{CDCl}_{3}\right) \square 7.12(\mathrm{~m}, 2 \mathrm{H}), 6.98(\mathrm{~m}, 4 \mathrm{H})$, $6.89(\mathrm{~m}, 2 \mathrm{H}), 6.79(\mathrm{~m}, 2 \mathrm{H}), 3.35(\mathrm{~s}, 1 \mathrm{H}), 3.00(\mathrm{~d}, J=2.6 \mathrm{~Hz}, 1 \mathrm{H}), 1.81(\mathrm{dd}, J=14.8,2.9 \mathrm{~Hz}, 1 \mathrm{H}), 1.66(\mathrm{dd}, J=$ $14.1,2.9 \mathrm{~Hz}, 1 \mathrm{H}), 1.63(\mathrm{dd}, J=14.8,2.5 \mathrm{~Hz}, 1 \mathrm{H}), 1.52(\mathrm{~d}, J=14.1 \mathrm{~Hz}, 1 \mathrm{H}), 1.41(\mathrm{~s}, 3 \mathrm{H}), 1.38(\mathrm{~s}, 3 \mathrm{H}), 1.34(\mathrm{~s}$, $3 \mathrm{H}), 0.90(\mathrm{~s}, 3 \mathrm{H}) ;{ }^{13} \mathrm{C}\left(125 \mathrm{MHz}, \mathrm{CDCl}_{3}\right) \square 148.4,136.8,133.1,128.4,127.6,126.6,126.4,125.3,79.1,71.1$, 54.5, 52.3, 36.9, 36.6, 35.4, 31.3, 27.9, 24.8; IR (thin film) 3505, 3030, 2951, $1445 \mathrm{~cm}^{-1}$; HRMS (ESI) $\mathrm{m} / z$ calcd for $\mathrm{C}_{22} \mathrm{H}_{28} \mathrm{NaOS}[\mathrm{M}+\mathrm{Na}]^{+}$363.1758, found 363.1751. Anal. Calcd for $\mathrm{C}_{22} \mathrm{H}_{28} \mathrm{OS}: \mathrm{C}, 77.60 ; \mathrm{H}, 8.29$. Found: $\mathrm{C}$, $77.41 ; \mathrm{H}, 8.43$.
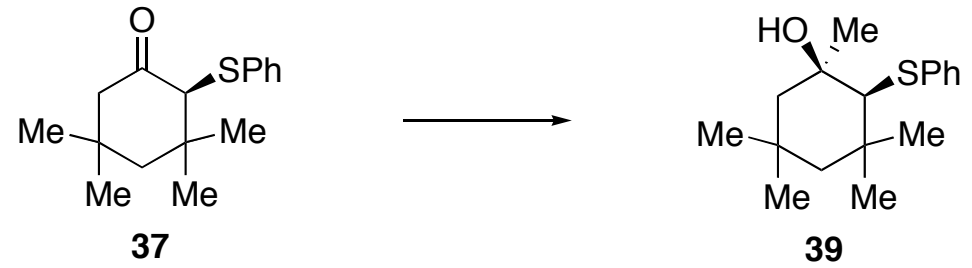

Cyclohexanol 39. The standard Grignard addition procedure was followed with $37(0.033 \mathrm{~g}, 0.13$ mmol) and methylmagnesium bromide $\left(0.10 \mathrm{~mL}, 0.30 \mathrm{mmol}, 3.0 \mathrm{M}^{\text {in }} \mathrm{Et}_{2} \mathrm{O}\right)$. GC and ${ }^{1} \mathrm{H}$ NMR spectroscopic analysis of the unpurified product detected only a single isomer was present. Purification of the resultant residue by silica gel chromatography (3:97 EtOAc:hexanes) yielded the product as a colorless oil $(0.027 \mathrm{~g}$, 77\%): ${ }^{1} \mathrm{H}$ NMR (400 MHz, $\left.\mathrm{CDCl}_{3}\right) \square 7.45(\mathrm{~m}, 2 \mathrm{H}), 7.28(\mathrm{~m}, 2 \mathrm{H}), 7.18(\mathrm{~m}, 1 \mathrm{H}), 2.89(\mathrm{~s}, 1 \mathrm{H}), 2.17(\mathrm{~d}, J=2.2$ $\mathrm{Hz}, 1 \mathrm{H}), 1.82(\mathrm{dd}, J=14.4,2.9 \mathrm{~Hz}, 1 \mathrm{H}), 1.57(\mathrm{dd}, J=14.0,2.9 \mathrm{~Hz}, 1 \mathrm{H}), 1.29(\mathrm{~m}, 2 \mathrm{H}), 1.32(\mathrm{~s}, 3 \mathrm{H}), 1.24(\mathrm{~s}$, $6 \mathrm{H}), 1.14(\mathrm{~s}, 3 \mathrm{H}), 0.89$ (s, 3H); ${ }^{13} \mathrm{C}\left(125 \mathrm{MHz}, \mathrm{CDCl}_{3}\right) \square 139.3,130.6,129.3,126.5,69.8,54.6,50.8,37.1,36.5$, 35.3, 33.6, 30.9, 27.7, 24.4, 22.7; IR (thin film) 3529, 2951, $1481 \mathrm{~cm}^{-1}$; HRMS (CI) $\mathrm{m} / z$ calcd for $\mathrm{C}_{17} \mathrm{H}_{25} \mathrm{~S}[\mathrm{M}-$ $\mathrm{OH}]^{+}$261.1677, found 261.1674. Anal. Calcd for $\mathrm{C}_{17} \mathrm{H}_{26} \mathrm{OS}: \mathrm{C}, 73.33$; H, 9.41. Found: C, 73.14; H, 9.65. 


\section{Stereochemical Proofs of Nucleophilic Substitution Products}

Relevant DPFGSE-nOe data (mixing time $2.0 \mathrm{~s}$ ): the peaks in the ${ }^{1} \mathrm{H}$ NMR spectra were assigned using ${ }^{1} \mathrm{H} /{ }^{1} \mathrm{H}$ COSY, ${ }^{1} \mathrm{H}$ NMR chemical shifts, and ${ }^{1} \mathrm{H}$ NMR coupling constants.<smiles>C=CC[C@]1(OC)CCCC[C@@H]1[SnH2]</smiles>

8

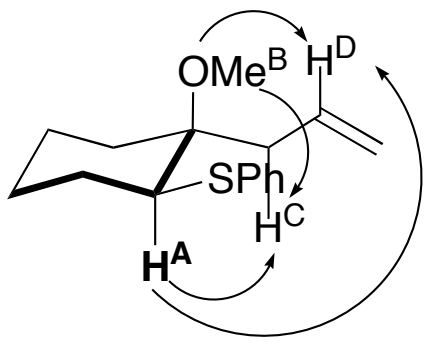

\section{Allyl Product (1,2-trans)}

$\mathbf{H}^{\mathbf{A}}$ irradiated: $\mathrm{H}^{\mathrm{C}}(1.1 \%), \mathrm{H}^{\mathrm{D}}(3.6 \%)$

OMe $^{\mathrm{B}}$ irradiated: $\mathrm{H}^{\mathrm{C}}(1.7 \%), \mathrm{H}^{\mathrm{D}}(0.4 \%)$

Note: The absence of nOe between $\mathrm{H}^{\mathrm{A}}$ and $\mathrm{OMe}^{\mathrm{B}}$ suggests 1,2-trans isomer. The splitting pattern of $\mathrm{H}^{\mathrm{A}}$ indicates that $\mathrm{H}^{\mathrm{A}}$ is axial: $\mathrm{dd}(J=11.1,4.1 \mathrm{~Hz})$.

Also, an nOe comparison was made between allyl sulfide $\mathbf{8}$ and meth-allyl sulfide 9, whose configuration was determined by X-ray crystallography.<smiles>C=CC[C@]1(OC)CCCC[C@H]1Cl</smiles>

$12 b$

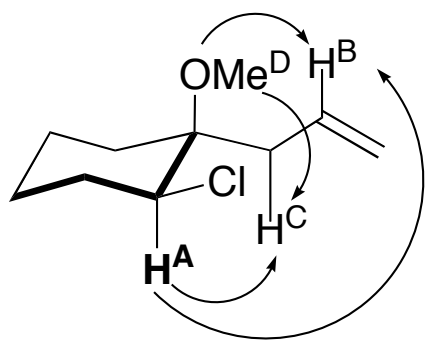

Chlorocyclohexane (1,2-trans, major)

$\mathbf{H}^{\mathrm{A}}$ irradiated: $\mathrm{H}^{\mathrm{B}}(0.4 \%), \mathrm{H}^{\mathrm{C}}(0.2 \%)$

OMe $^{\mathbf{B}}$ irradiated: $\mathrm{HC}$ (

Note: The splitting pattern of $\mathrm{H}^{\mathrm{A}}$ indicates that $\mathrm{H}^{\mathrm{A}}$ is axial: $\mathrm{dd}(J=10.7,3.8 \mathrm{~Hz})$. The nOe between $\mathrm{H}^{\mathrm{A}}$ and $\mathrm{H}^{\mathrm{B}}$ and $\mathrm{H}^{\mathrm{C}}$ suggests the 1,2-trans isomer.<smiles>C=CC[C@]1(OC)CCCC[C@H]1Cl</smiles>

$13 b$
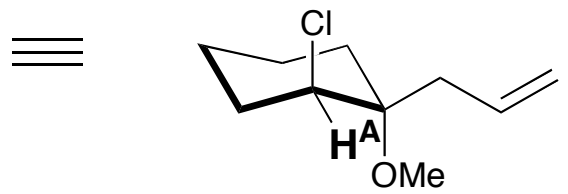

Chlorocyclohexane (1,2-cis, minor)

Note: The splitting pattern of $\mathrm{H}^{\mathrm{A}}$ indicates that $\mathrm{H}^{\mathrm{A}}$ is equatorial (multiplet), 
suggests 1,2-cis isomer. This can be further confirmed if compared to major isomer.<smiles>C=CCC1CCCCC1(OC)OC</smiles><smiles>C#CCCC#C</smiles>

12c

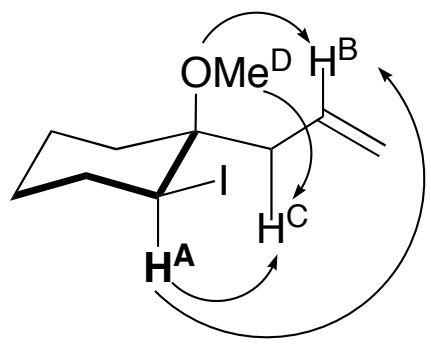

\section{Iodocyclohexane (1,2-trans)}

$\mathbf{H}^{\mathrm{A}}$ irradiated: $\mathrm{H}^{\mathrm{B}}(0.4 \%), \mathrm{H}^{\mathrm{C}}(0.3 \%)$

OMe $^{\mathrm{D}}$ irradiated: $\mathrm{H}^{\mathrm{B}}(0.1 \%), \mathrm{H}^{\mathrm{C}}(0.3 \%)$

Note: The absence of nOe between $\mathrm{H}^{\mathrm{A}}$ and $\mathrm{OMe}^{\mathrm{B}}$ suggests 1,2-trans isomer. The splitting pattern of $\mathrm{H}^{\mathrm{A}}$ indicates that $\mathrm{H}^{\mathrm{A}}$ is axial: $\mathrm{dd}(J=10.2,4.2 \mathrm{~Hz})$<smiles>C=CC[C@]1(O)CCCC[C@@H]1Oc1ccccc1</smiles>

12d

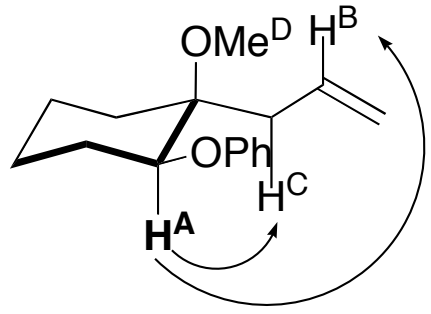

\section{Phenoxycyclohexane (1,2-trans, major)}

$\mathbf{H}^{\mathrm{A}}$ irradiated: $\mathrm{H}^{\mathrm{B}}(0.6 \%), \mathrm{H}^{\mathrm{C}}(0.9 \%)$

Note: The splitting pattern of $\mathrm{H}^{\mathrm{A}}$ indicates that $\mathrm{H}^{\mathrm{A}}$ is axial:

dd $(J=10.5,3.7 \mathrm{~Hz})$. The nOe between $\mathrm{H}^{\mathrm{A}}$ and $\mathrm{H}^{\mathrm{B}}, \mathrm{H}^{\mathrm{C}}$ suggests the 1,2-trans isomer.<smiles>C=CC[C@]1(OC)CCCCC1Oc1ccccc1</smiles>

13d

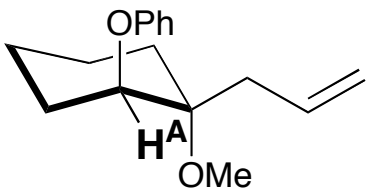

\section{Phenoxycyclohexane (1,2-cis, minor)}

Note: The splitting pattern of $\mathrm{H}^{\mathrm{A}}$ indicates that $\mathrm{H}^{\mathrm{A}}$ is equatorial: $\mathrm{t}(J=3.6 \mathrm{~Hz})$, suggests 1,2-cis isomer. This can be further confirmed if compared to the major isomer. 
<smiles>C=CC[C@]1(OC)CC[C@@H](C(C)(C)C)C[C@H]1c1ccccc1</smiles>

20

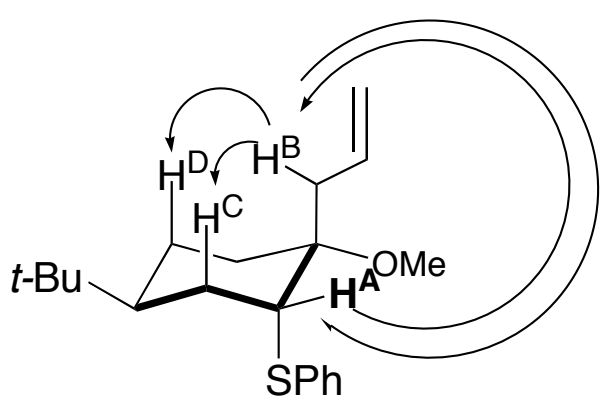

Allyl Product (1,2-trans)

$\mathbf{H}^{\mathrm{A}}$ irradiated: $\mathrm{H}^{\mathrm{B}}(1.9 \%), \mathrm{H}^{\mathrm{C}}(2.4 \%)$

$\mathbf{H}^{\mathrm{B}}$ irradiated: $\mathrm{H}^{\mathrm{A}}(7.0 \%), \mathrm{H}^{\mathrm{C}}$ and $\mathrm{H}^{\mathrm{D}}(11.2 \%)$

Note: The presence of nOe between $\mathrm{H}^{\mathrm{B}}$ and $\mathrm{H}^{\mathrm{C}}$, and $\mathrm{H}^{\mathrm{D}}$ suggests that the allyl group is axial. The splitting pattern of $\mathrm{H}^{\mathrm{A}}$ is indicative of an equatorial proton and confirms this structure as 1,2-trans.
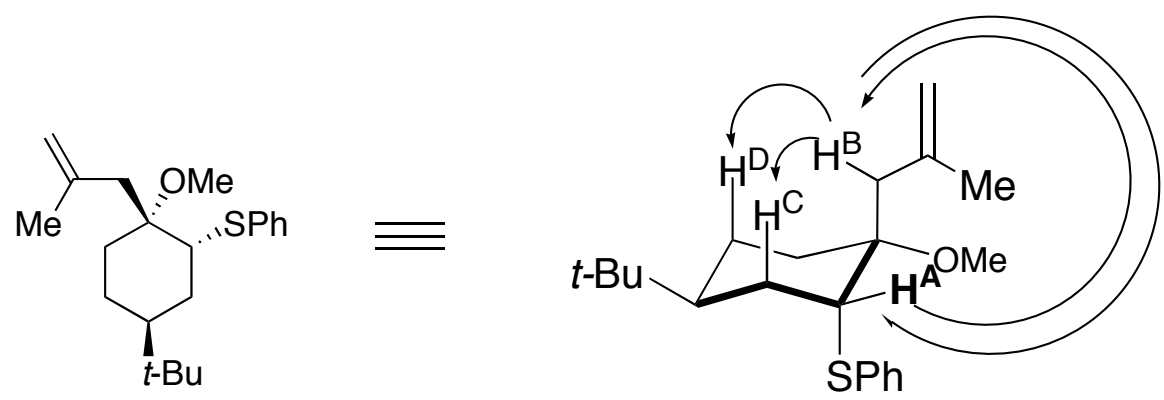

21

\section{Methallyl Product (1,2-trans)}

$\mathbf{H}^{\mathrm{A}}$ irradiated: $\mathrm{H}^{\mathrm{B}}(2.5 \%), \mathrm{H}^{\mathrm{C}}(3.9 \%)$

$\mathbf{H}^{\mathrm{B}}$ irradiated: $\mathrm{H}^{\mathrm{A}}(9.6 \%), \mathrm{H}^{\mathrm{C}}$ and $\mathrm{H}^{\mathrm{D}}(16.4 \%)$

Note: The presence of nOe between $\mathrm{H}^{\mathrm{B}}$ and $\mathrm{H}^{\mathrm{C}}, \mathrm{H}^{\mathrm{D}}$ suggests that the methallyl group is axial. The splitting pattern of $\mathrm{H}^{\mathrm{A}}$ is indicative of an equatorial proton and confirms this structure as 1,2-trans.

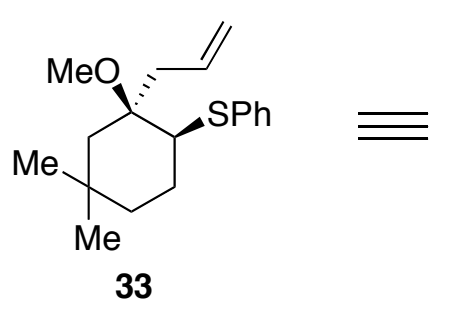

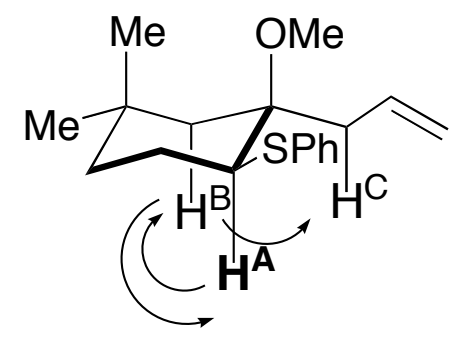
Allyl Product (1,2-trans)
$\mathbf{H}^{\mathrm{A}}$ irradiated: $\mathrm{H}^{\mathrm{B}}(0.7 \%), \mathrm{H}^{\mathrm{C}}(0.4 \%)$
$\mathbf{H}^{\mathrm{B}}$ irradiated: $\mathrm{H}^{\mathrm{A}}(1.5 \%), \mathrm{H}^{\mathrm{C}}(0.5 \%)$ 
Note: The absence of nOe between $\mathrm{H}^{\mathrm{B}}$ and $\mathrm{SPh}$ indicates 1,2-trans. The splitting pattern of $\mathrm{H}^{\mathrm{A}}$ indicates that $\mathrm{H}^{\mathrm{A}}$ is axial: $\mathrm{dd}(J=12.2,4.2 \mathrm{~Hz})$ and confirms this structure as 1,2-trans.

The stereochemistry of $\mathbf{3 1}$ was assigned by conversion of $\mathbf{3 1}$ to $\mathbf{S - 1 0}$, whose configuration was determined by analysis of nOe data.
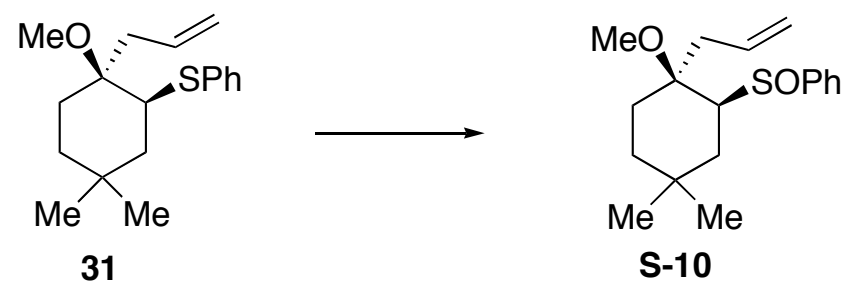

Sulfoxide S-10. ${ }^{26}$ To a cooled $\left(0{ }^{\circ} \mathrm{C}\right)$ solution of 4,4-dimethyl allyl sulfide $31(0.018 \mathrm{~g}, 0.060 \mathrm{mmol})$ in $0.6 \mathrm{~mL}(0.1 \mathrm{M})$ was added a solution of mCPBA $(0.011 \mathrm{~g}, 0.060 \mathrm{mmol})$ in $0.5 \mathrm{~mL}$ of $\mathrm{CH}_{2} \mathrm{Cl}_{2}$. The reaction mixture was allowed to warm to $23^{\circ} \mathrm{C}$. After stirring for $12 \mathrm{~h}$, sodium bisulfite $(0.010 \mathrm{~g})$ was added, and the reaction mixture was poured into a separatory funnel with $\mathrm{H}_{2} \mathrm{O}(2 \mathrm{~mL})$. The aqueous layer was extracted with $\mathrm{CH}_{2} \mathrm{Cl}_{2}(2 \times 3 \mathrm{~mL})$. The combined organic layers were washed with a saturated sodium chloride solution (5 $\mathrm{mL})$, dried $\left(\mathrm{Na}_{2} \mathrm{SO}_{4}\right)$, and concentrated in vacuo. Purification of the resultant residue by silica gel chromatography (10:90 to 20:80 EtOAc:hexanes) yielded the product as a white solid (0.016 g, 88\%): $\mathrm{mp} 100$ ${ }^{\circ} \mathrm{C} ;{ }^{1} \mathrm{H}$ NMR (500 MHz, $\left.\mathrm{CDCl}_{3}\right) \square 7.55(\mathrm{~m}, 2 \mathrm{H}), 7.47(\mathrm{~m}, 2 \mathrm{H}), 7.42(\mathrm{~m}, 1 \mathrm{H}), 5.82,(\mathrm{~m}, 1 \mathrm{H}), 5.29(\mathrm{~m}, 2 \mathrm{H}), 3.35$ (s, $3 \mathrm{H}), 3.00(\mathrm{dd}, J=13.9,8.9 \mathrm{~Hz}, 1 \mathrm{H}), 2.75(\mathrm{dd}, J=13.9,5.9 \mathrm{~Hz}, 1 \mathrm{H}), 2.58(\mathrm{dd}, J=12.9,4.1 \mathrm{~Hz}, 1 \mathrm{H}), 1.97(\mathrm{t}$, $J=13.1 \mathrm{~Hz}, 1 \mathrm{H}), 1.84(\mathrm{dt}, J=14.4,3.1 \mathrm{~Hz}, 1 \mathrm{H}), 1.53(\mathrm{td}, J=14.2,3.1 \mathrm{~Hz}, 1 \mathrm{H}), 1.45(\mathrm{td}, J=13.3,3.3 \mathrm{~Hz}, 1 \mathrm{H})$, $1.11(\mathrm{ddd}, J=11.2,5.4,2.5 \mathrm{~Hz}, 1 \mathrm{H}), 0.93(\mathrm{~s}, 3 \mathrm{H}), 0.89(\mathrm{ddd}, J=13.3,4.0,2.4 \mathrm{~Hz}, 1 \mathrm{H}), 0.60(\mathrm{~s}, 3 \mathrm{H}) ;{ }^{13} \mathrm{C}$ NMR $\left(125 \mathrm{MHz}, \mathrm{CDCl}_{3}\right) \square 144.9,133.9,130.2,129.1,124.5,120.2,76.8,66.3,48.9,40.8,33.5,32.6,30.4,28.4$, 26.7, 24.4; IR (thin film) 3078, 2952, 1443, $1082 \mathrm{~cm}^{-1}$; HRMS (ESI) $\mathrm{m} / z$ calcd for $\mathrm{C}_{18} \mathrm{H}_{26} \mathrm{NaO}_{2} \mathrm{~S}[\mathrm{M}+\mathrm{Na}]^{+}$ 329.1551, found 329.1546. Anal. Calcd for $\mathrm{C}_{18} \mathrm{H}_{26} \mathrm{O}_{2} \mathrm{~S}: \mathrm{C}, 70.54 ; \mathrm{H}, 8.55$. Found: C, 70.90; H, 8.31.
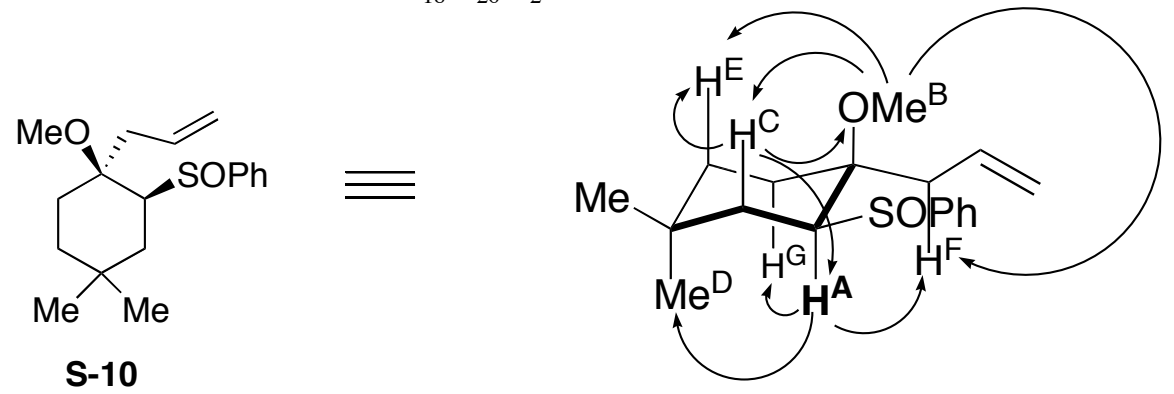

Sulfoxide (1,2-trans)

$\mathbf{H}^{\mathrm{A}}$ irradiated: $\mathrm{Me}^{\mathrm{D}}(2.1 \%), \mathrm{H}^{\mathrm{F}}(0.7 \%), \mathrm{H}^{\mathrm{G}}(1.2 \%)$

OMe $^{\mathrm{B}}$ irradiated: $\mathrm{H}^{\mathrm{C}}(0.1 \%), \mathrm{H}^{\mathrm{E}}(0.3 \%), \mathrm{H}^{\mathrm{F}}(0.6 \%)$

$\mathbf{H}^{\mathrm{C}}$ irradiated: $\mathrm{H}^{\mathrm{A}}(0.1 \%), \mathrm{OMe}^{\mathrm{B}}(0.2 \%), \mathrm{H}^{\mathrm{E}}(0.2 \%)$

Note: The absence of nOe between $\mathrm{H}^{\mathrm{A}}$ and $\mathrm{OMe}^{\mathrm{B}}$ indicates that these substituents are trans with respect to each other. This nOe analysis confirms the stereochemistry as the trans isomer. 
The stereochemistry of $\mathbf{2 2}$ was assigned by conversion of $\mathbf{2 2}$ to S-11, whose structure was determined by X-ray crystallography.<smiles></smiles>

22

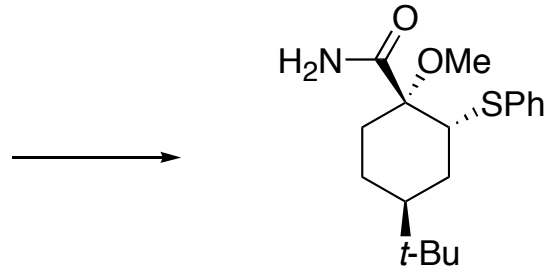

S-11

Amide S-11. ${ }^{27}$ To a solution of $22(0.022 \mathrm{~g}, 0.072 \mathrm{mmol})$ in $0.72 \mathrm{~mL}$ of EtOH was added a solution of $\mathrm{K}_{2} \mathrm{CO}_{3}(0.110 \mathrm{~g}, 1.44 \mathrm{mmol})$ in $0.20 \mathrm{~mL}$ of $\mathrm{H}_{2} \mathrm{O}$ followed by $0.25 \mathrm{~mL}$ of $30 \% \mathrm{H}_{2} \mathrm{O}_{2}$. After $16 \mathrm{~h}$ at $23{ }^{\circ} \mathrm{C}$, the reaction mixture was cooled $\left(0{ }^{\circ} \mathrm{C}\right)$ and $\mathrm{NaS}_{2} \mathrm{O}_{3} \cdot 7 \mathrm{H}_{2} \mathrm{O}$ was added slowly until bubbling ceased. The solution was concentrated in vacuo, and the residue was extracted with $\mathrm{CH}_{2} \mathrm{Cl}_{2}(3 \times 3 \mathrm{~mL})$. The combined organic layers were dried $\left(\mathrm{MgSO}_{4}\right)$ and concentrated in vacuo. Purification of the resultant residue by silica gel chromatography (10:90 EtOAc:hexanes) yielded the product as a white solid $(0.018 \mathrm{~g}, 78 \%)$. X-ray quality crystals were grown from $\mathrm{CH}_{2} \mathrm{Cl}_{2}$ in which slow evaporation provided the crystal: mp $138{ }^{\circ} \mathrm{C} ;{ }^{1} \mathrm{H}$ NMR (400 $\left.\mathrm{MHz}, \mathrm{CDCl}_{3}\right) \square 7.45(\mathrm{~m}, 2 \mathrm{H}), 7.29(\mathrm{~m}, 3 \mathrm{H}), 6.22$ (broad s, $\left.1 \mathrm{H}\right), 5.43$ (broad s, $\left.1 \mathrm{H}\right), 4.11(\mathrm{~m}, 1 \mathrm{H}), 3.35(\mathrm{~s}, 3 \mathrm{H})$, $1.97(\mathrm{~m}, 2 \mathrm{H}), 1.81(\mathrm{~m}, 3 \mathrm{H}), 1.67(\mathrm{~m}, 1 \mathrm{H}), 1.27(\mathrm{~m}, 1 \mathrm{H}), 0.76(\mathrm{~s}, 9 \mathrm{H}) ;{ }^{13} \mathrm{C}$ NMR $\left(125 \mathrm{MHz}, \mathrm{CDCl}_{3}\right) \mathrm{1}$ 175.2, 135.0, 133.3, 129.2, 127.5, 82.0, 51.8, 50.9, 40.8, 32.4, 32.2, 29.2, 27.8, 24.1; IR (thin film) 3300, 3056, 2958, $2869,1673 \mathrm{~cm}^{-1}$; HRMS (ESI) $\mathrm{m} / z$ calcd for $\mathrm{C}_{18} \mathrm{H}_{27} \mathrm{NaNO}_{2} \mathrm{~S}\left[\mathrm{M}+\mathrm{Na}^{+}\right.$344.1660, found 344.1667. Anal. Calcd for $\mathrm{C}_{18} \mathrm{H}_{27} \mathrm{NO}_{2} \mathrm{~S}$ : C, 67.25; H, 8.47; N, 4.36. Found: C, 67.22; H, 8.54; N, 4.25.

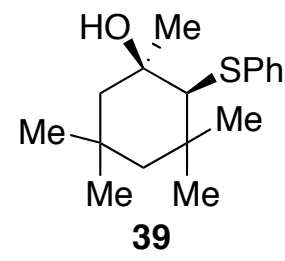

Sulfoxide S-12. ${ }^{27}$ To a cooled $\left(0{ }^{\circ} \mathrm{C}\right)$ solution of $39(0.043 \mathrm{~g}, 0.15 \mathrm{mmol})$ in $1.50 \mathrm{~mL}$ of $\mathrm{Et}_{2} \mathrm{O}(0.10 \mathrm{M})$ was added a solution of mCPBA $(0.080 \mathrm{~g}, 0.46 \mathrm{mmol})$ in $0.6 \mathrm{~mL}$ of $\mathrm{Et}_{2} \mathrm{O}(0.80 \mathrm{M})$. After stirring for $30 \mathrm{~min}$ at $0{ }^{\circ} \mathrm{C}$, sodium bisulfite $(0.020 \mathrm{~g})$ was added and the reaction mixture was poured into a separatory funnel with $\mathrm{H}_{2} \mathrm{O}(5 \mathrm{~mL})$. The aqueous layer was extracted with $\mathrm{CH}_{2} \mathrm{Cl}_{2}(2 \times 5 \mathrm{~mL})$. The combined organic layers were washed with a saturated sodium chloride solution $(10 \mathrm{~mL})$, dried $\left(\mathrm{Na}_{2} \mathrm{SO}_{4}\right)$, and concentrated in vacuo. Purification of the resultant residue by silica gel chromatography (5:95 to 10:90 EtOAc:hexanes) yielded the product as a white solid $(0.036 \mathrm{~g}, 84 \%)$. X-ray quality crystals were grown from a 5:1 mixture of hexanes and $\mathrm{CH}_{2} \mathrm{Cl}_{2}$ in which slow evaporation provided the crystal: mp $135-138{ }^{\circ} \mathrm{C} ;{ }^{1} \mathrm{H} \mathrm{NMR}\left(500 \mathrm{MHz}, \mathrm{CDCl}_{3}\right) \square 7.60$ (d, $J=7.4 \mathrm{~Hz}, 2 \mathrm{H}), 7.52(\mathrm{t}, J=7.7 \mathrm{~Hz}, 2 \mathrm{H}), 7.44(\mathrm{t}, J=7.2 \mathrm{~Hz}, 1 \mathrm{H}), 5.00(\mathrm{~d}, J=2.6 \mathrm{~Hz}, 1 \mathrm{H}), 2.42(\mathrm{~s}, 1 \mathrm{H}), 1.78(\mathrm{~s}$, $3 \mathrm{H}), 1.55(\mathrm{~m}, 1 \mathrm{H}), 1.32(\mathrm{~s}, 3 \mathrm{H}), 1.27(\mathrm{~s}, 3 \mathrm{H}), 1.26(\mathrm{~m}, 2 \mathrm{H}), 1.12(\mathrm{~s}, 3 \mathrm{H}), 1.09(1 \mathrm{H}), 0.84(\mathrm{~s}, 3 \mathrm{H}) ;{ }^{13} \mathrm{C}$ NMR $(125$ $\left.\mathrm{MHz}, \mathrm{CDCl}_{3}\right) \square$ 145.6, 130.0, 129.4, 124.1, 78.0, 77.7, 54.7, 54.1, 36.3, 36.2, 35.2, 32.4, 31.1, 27.8, 24.8; IR (thin film) 3377, 2919, 2868, $1410 \mathrm{~cm}^{-1}$; HRMS (ESI) $\mathrm{m} / z$ calcd for $\mathrm{C}_{17} \mathrm{H}_{27} \mathrm{O}_{2} \mathrm{~S}[\mathrm{M}+\mathrm{H}]^{+} 295.1732$, found 295.1737. Anal. Calcd for $\mathrm{C}_{17} \mathrm{H}_{26} \mathrm{SO}$ : C, 69.34; H, 8.90. Found: C, 69.89; H, 9.13. 
X-Ray Crystallography Data for Nucleophilic Substitution Products: 9, 10, S-11, 18, 38, and S-12. See Analytical Section for additional X-ray crystal data.
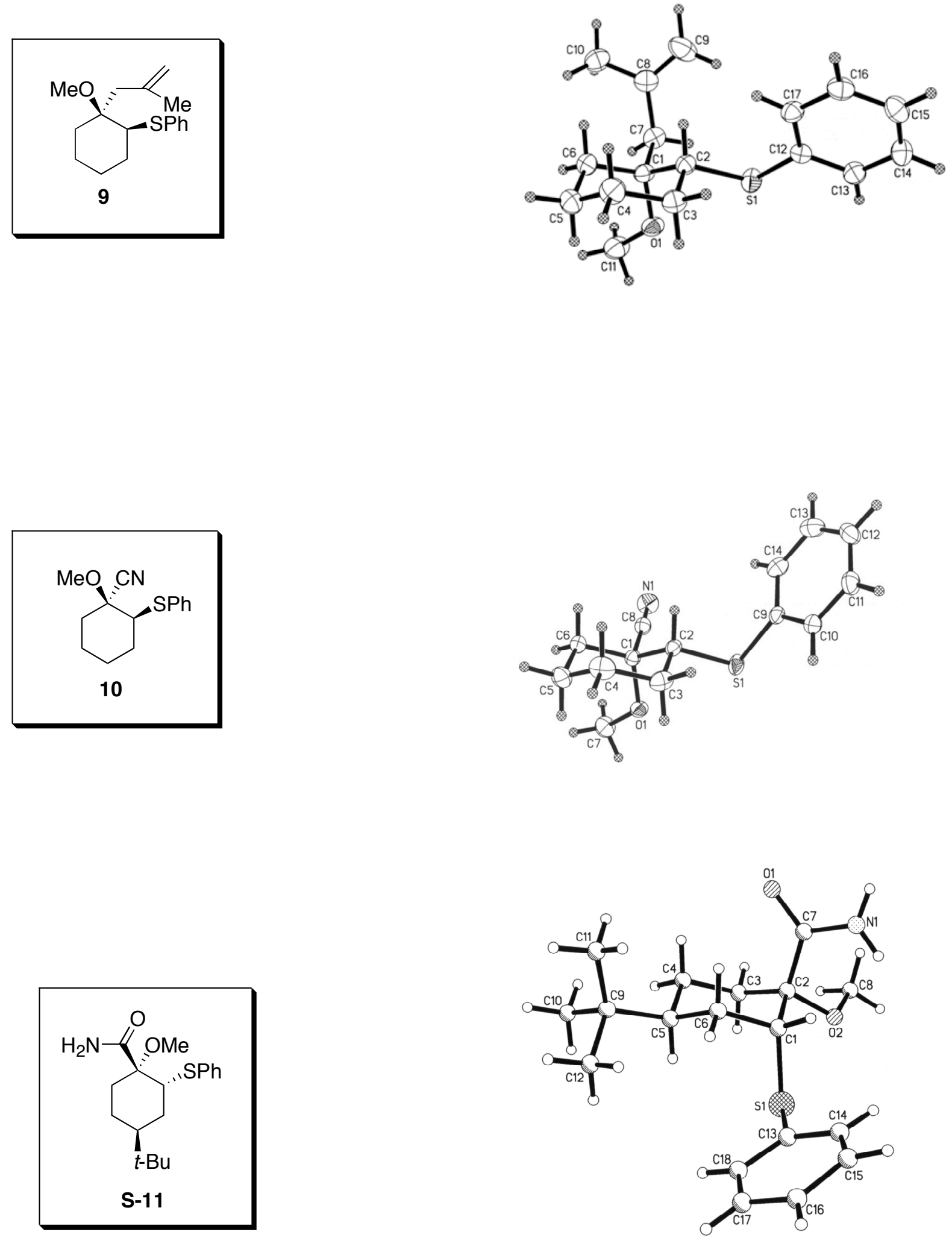

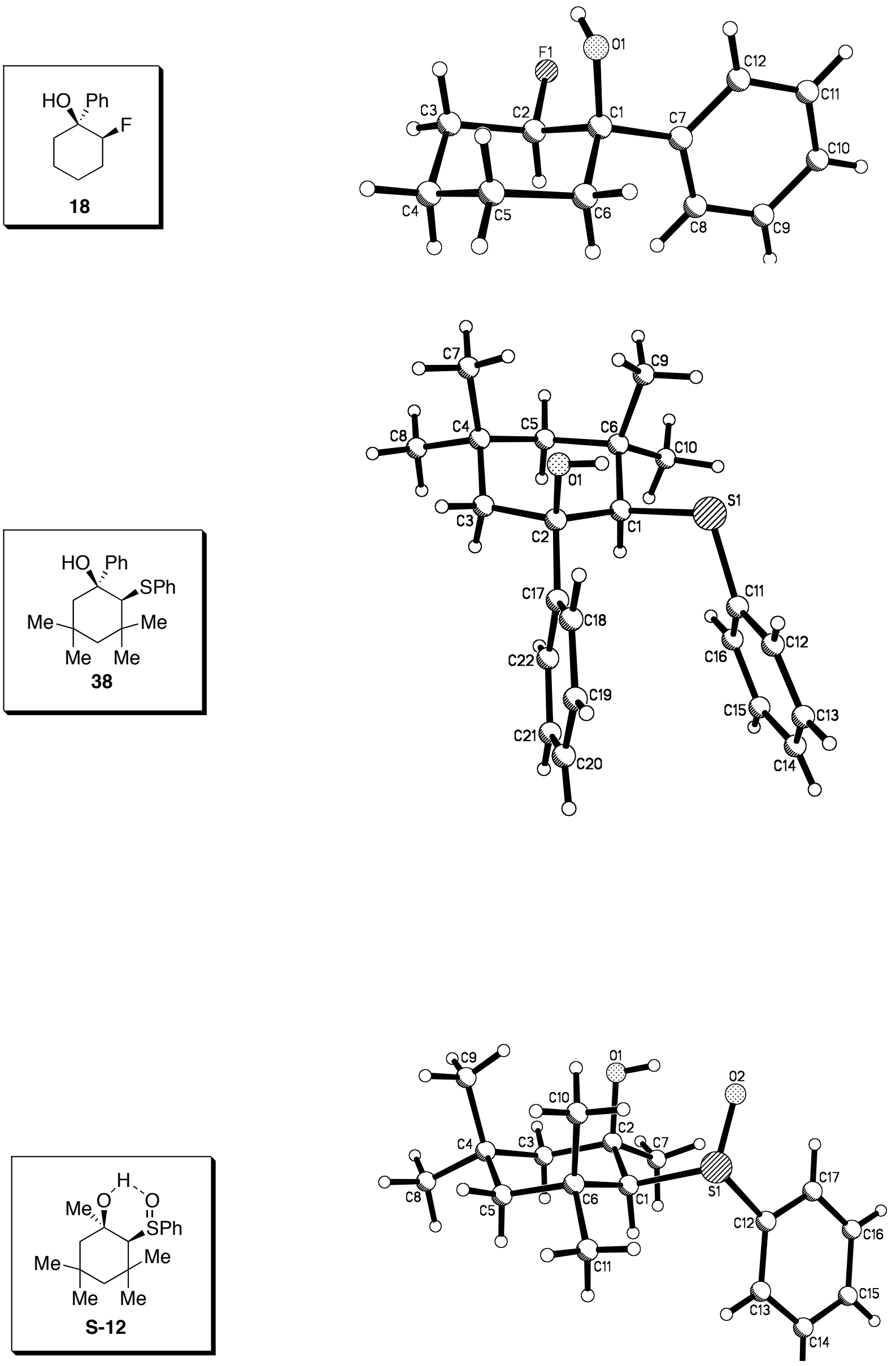


\section{Bibliography}

(1) Pangborn, A. B.; Giardello, M. A.; Grubbs, R. H.; Rosen, R. K.; Timmers, F. J. Organometallics 1996, 15, 1518-1520.

(2) Overman, L. E.; Robichaud, A. J. J. Am. Chem. Soc. 1989, 111, 300-308.

(3) Trost, B. M.; Salzmann, T. N.; Hiroi, K. J. Am. Chem. Soc. 1976, 98, 4887-4902.

(4) De Amici, M.; De Micheli, C.; Molteni, G.; Pitre, D.; Carrea, G.; Riva, S.; Spezia, S.; Zetta, L. J. Org. Chem. 1991, 56, 67-72.

(5) Mursakulov, I. G.; Guseinov, M. M.; Kasumov, N. K.; Zefirov, N. S.; Samoshin, V. V.; Chalenko, E. G. Tetrahedron 1982, 38, 2213-2220.

(6) Tanikaga, R.; Nishikawa, T.; Tomita, N. Bull. Chem. Soc. Jpn. 1999, 72, 1057-1062.

(7) Dess, D. B.; Martin, J. C. J. Org. Chem. 1983, 48, 4156-4158.

(8) Meyer, S. D.; Schreiber, S. L. J. Org. Chem. 1994, 59, 7549-7552.

(9) Bordwell, F. G.; Wellman, K. M. J. Org. Chem. 1963, 28, 1347-1352.

(10) Reid, E. B.; Gompf, T. E. J. Org. Chem. 1953, 18, 661-663.

(11) Carreno, M. C.; Garcia Ruano, J. L.; Martin, A. M.; Pedregal, C.; Rodriguez, J. H.; Rubio, A.; Sanchez, J.; Solladie, G. J. Org. Chem. 1990, 55, 2120-2128.

(12) Fuchigami, T.; Shimojo, M.; Konno, A. J. Org. Chem. 1995, 60, 3459-3464.

(13) Liphshutz, B. H.; Ellsworth, E. L.; Siahaan, T. J. J. Am. Chem. Soc. 1989, 111, 1351-1358.

(14) House, H. O.; Fischer, W. F. J. Org. Chem. 1968, 33, 949-956.

(15) Bartel, S.; Bohlmann, F. Tetrahedron Lett. 1989, 30, 685-688.

(16) Wolker, D.; Haufe, G. J. Org. Chem. 2002, 67, 3015-3021.

(17) Bruns, S.; Haufe, G. J. Fluorine. Chem. 2000, 104, 247-254.

(18) Welch, J. T.; Seper, K. W. J. Org. Chem. 1988, 53, 2991-2999.

(19) Masilamani, D.; Manahan, E., H.; Vitrone, J.; Rogic, M. M. J. Org. Chem. 1983, 48, 4918-4931.

(20) Torii, S.; Inokuchi, T.; Misima, S.; Kobayashi, T. J. Org. Chem. 1980, 45, 2731-2735.

(21) Horiuchi, C. A.; Kiji, S. Bull. Chem. Soc. Jpn. 1997, 70, 421-426.

(22) D'Auria, M.; D'Onofrio, F.; Piancetelli, G.; Scettri, A. Synth. Commun. 1982, 12, 1127-1138.

(23) Iranpoor, N.; Firouzabadi, H.; Safavi, A.; Shekarriz, M. Synth. Commun. 2002, 32, 2287-2293.

(24) Koreeda, M.; Patel, P. D.; Brown, L. J. Org. Chem. 1985, 50, 5910-5912.

(25) Williams, D. R.; Gaston, R. D. Tetrahedron Lett. 1986, 27, 1485-1488.

(26) Kozikowski, A. P.; Li, C. J. Org. Chem. 1987, 52, 3541-3552.

(27) Willson, T. M.; Kocienski, P.; Jarowicki, K.; Isaac, K.; Hitchcock, P. M.; Faller, A.; Campbell, S. F. Tetrahedron 1990, 46, 1767-1782. 


\section{Analytical Data}

\section{A. X-Ray Crystal Data}

Table 1. Crystal data and structure refinement for Allyl Product 9.

Identification code

Empirical formula

Formula weight

Temperature

Wavelength

Crystal system

Space group

Unit cell dimensions

Volume

\section{Z}

Density (calculated)

Absorption coefficient

$\mathrm{F}(000)$

Crystal size

Theta range for data collection

Index ranges

Reflections collected

Independent reflections

Completeness to theta $=28.29^{\circ}$

Absorption correction

Max. and min. transmission

Refinement method

Data / restraints / parameters

Goodness-of-fit on $\mathrm{F}^{2}$

Final $\mathrm{R}$ indices [I $>2 \operatorname{sigma}(\mathrm{I})]$

$\mathrm{R}$ indices (all data)
Allyl Product 9

$\mathrm{C}_{17} \mathrm{H}_{24} \mathrm{O} \mathrm{S}$

276.42

163(2) K

$0.71073 \AA$

Orthorhombic

$\mathrm{P} 2{ }_{1} 2_{1} 2_{1}$

$\mathrm{a}=8.1328(4) \AA \quad \square=90^{\circ}$.

$\mathrm{b}=9.4467(4) \AA \quad \square=90^{\circ}$.

$\mathrm{c}=20.3760(9) \AA \quad \square=90^{\circ}$.

$1565.45(12) \AA^{3}$

4

$1.173 \mathrm{Mg} / \mathrm{m}^{3}$

$0.198 \mathrm{~mm}^{-1}$

600

$0.46 \times 0.45 \times 0.26 \mathrm{~mm}^{3}$

2.38 to $28.29^{\circ}$.

-10 h $10,-12$ k 12, -26 127

16003

$3760[\mathrm{R}(\mathrm{int})=0.0239]$

$99.0 \%$

Semi-empirical from equivalents

0.9503 and 0.9144

Full-matrix least-squares on $\mathrm{F}^{2}$

3760 / 0 / 268

1.058

$\mathrm{R} 1=0.0276, \mathrm{wR} 2=0.0728$

$\mathrm{R} 1=0.0292, w R 2=0.0738$

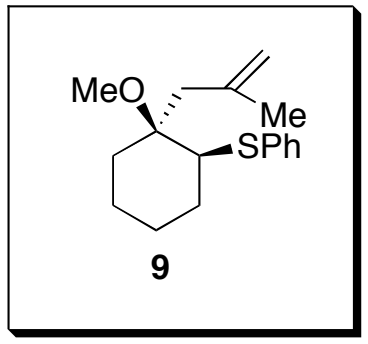


Absolute structure parameter

Largest diff. peak and hole
$0.53(5)$

0.274 and -0.136 e. $\AA^{-3}$ 
Table 2. Atomic coordinates $\left(\times 10^{4}\right)$ and equivalent isotropic displacement parameters $\left(\AA^{2} \times 10^{3}\right)$ for kaw49. $U(e q)$ is defined as one third of the trace of the orthogonalized Uij tensor.

\begin{tabular}{lrrrr}
\hline & $\mathrm{x}$ & $\mathrm{y}$ & $\mathrm{z}$ & $\mathrm{U}(\mathrm{eq})$ \\
\hline $\mathrm{S}(1)$ & $4013(1)$ & $316(1)$ & $1110(1)$ & $31(1)$ \\
$\mathrm{O}(1)$ & $4165(1)$ & $2591(1)$ & $2053(1)$ & $33(1)$ \\
$\mathrm{C}(1)$ & $2621(1)$ & $2626(1)$ & $1710(1)$ & $26(1)$ \\
$\mathrm{C}(2)$ & $3061(1)$ & $2055(1)$ & $1019(1)$ & $24(1)$ \\
$\mathrm{C}(3)$ & $4208(2)$ & $3051(1)$ & $644(1)$ & $31(1)$ \\
$\mathrm{C}(4)$ & $3488(2)$ & $4538(2)$ & $590(1)$ & $37(1)$ \\
$\mathrm{C}(5)$ & $3128(2)$ & $5124(2)$ & $1273(1)$ & $37(1)$ \\
$\mathrm{C}(6)$ & $1953(2)$ & $4137(1)$ & $1636(1)$ & $31(1)$ \\
$\mathrm{C}(7)$ & $1387(2)$ & $1659(2)$ & $2076(1)$ & $31(1)$ \\
$\mathrm{C}(8)$ & $-341(2)$ & $1580(2)$ & $1803(1)$ & $33(1)$ \\
$\mathrm{C}(9)$ & $-725(2)$ & $682(2)$ & $1324(1)$ & $51(1)$ \\
$\mathrm{C}(10)$ & $-1610(2)$ & $2498(2)$ & $2116(1)$ & $43(1)$ \\
$\mathrm{C}(11)$ & $4251(2)$ & $3294(2)$ & $2670(1)$ & $45(1)$ \\
$\mathrm{C}(12)$ & $3994(1)$ & $-402(1)$ & $310(1)$ & $24(1)$ \\
$\mathrm{C}(13)$ & $5026(2)$ & $-1565(1)$ & $215(1)$ & $30(1)$ \\
$\mathrm{C}(14)$ & $5071(2)$ & $-2251(2)$ & $-386(1)$ & $34(1)$ \\
$\mathrm{C}(15)$ & $4083(2)$ & $-1788(2)$ & $-900(1)$ & $35(1)$ \\
$\mathrm{C}(16)$ & $3076(2)$ & $-631(2)$ & $-811(1)$ & $32(1)$ \\
$\mathrm{C}(17)$ & $3021(2)$ & $70(1)$ & $-209(1)$ & $28(1)$ \\
& & & & \\
\hline
\end{tabular}


Table 3. Bond lengths $[\AA]$ and angles $\left[{ }^{\circ}\right]$ for kaw49.

\begin{tabular}{ll}
\hline $\mathrm{S}(1)-\mathrm{C}(12)$ & $1.7661(11)$ \\
$\mathrm{S}(1)-\mathrm{C}(2)$ & $1.8251(12)$ \\
$\mathrm{O}(1)-\mathrm{C}(11)$ & $1.4233(16)$ \\
$\mathrm{O}(1)-\mathrm{C}(1)$ & $1.4382(14)$ \\
$\mathrm{C}(1)-\mathrm{C}(6)$ & $1.5345(18)$ \\
$\mathrm{C}(1)-\mathrm{C}(7)$ & $1.5482(17)$ \\
$\mathrm{C}(1)-\mathrm{C}(2)$ & $1.5501(16)$ \\
$\mathrm{C}(2)-\mathrm{C}(3)$ & $1.5301(17)$ \\
$\mathrm{C}(3)-\mathrm{C}(4)$ & $1.526(2)$ \\
$\mathrm{C}(4)-\mathrm{C}(5)$ & $1.526(2)$ \\
$\mathrm{C}(5)-\mathrm{C}(6)$ & $1.526(2)$ \\
$\mathrm{C}(7)-\mathrm{C}(8)$ & $1.5135(19)$ \\
$\mathrm{C}(8)-\mathrm{C}(9)$ & $1.330(2)$ \\
$\mathrm{C}(8)-\mathrm{C}(10)$ & $1.492(2)$ \\
$\mathrm{C}(12)-\mathrm{C}(17)$ & $1.3931(16)$ \\
$\mathrm{C}(12)-\mathrm{C}(13)$ & $1.3955(18)$ \\
$\mathrm{C}(13)-\mathrm{C}(14)$ & $1.3854(18)$ \\
$\mathrm{C}(14)-\mathrm{C}(15)$ & $1.390(2)$ \\
$\mathrm{C}(15)-\mathrm{C}(16)$ & $1.378(2)$ \\
$\mathrm{C}(16)-\mathrm{C}(17)$ & $1.3953(18)$ \\
$\mathrm{C}(12)-\mathrm{S}(1)-\mathrm{C}(2)$ & $104.39(5)$ \\
$\mathrm{C}(11)-\mathrm{O}(1)-\mathrm{C}(1)$ & $117.50(10)$ \\
$\mathrm{O}(1)-\mathrm{C}(1)-\mathrm{C}(6)$ & $112.22(10)$ \\
$\mathrm{O}(1)-\mathrm{C}(1)-\mathrm{C}(7)$ & $108.57(10)$ \\
$\mathrm{C}(6)-\mathrm{C}(1)-\mathrm{C}(7)$ & $111.54(10)$ \\
$\mathrm{O}(1)-\mathrm{C}(1)-\mathrm{C}(2)$ & $103.41(9)$ \\
$\mathrm{C}(6)-\mathrm{C}(1)-\mathrm{C}(2)$ & $108.44(10)$ \\
$\mathrm{C}(7)-\mathrm{C}(1)-\mathrm{C}(2)$ & $112.43(10)$ \\
$\mathrm{C}(3)-\mathrm{C}(2)-\mathrm{C}(1)$ & $112.39(10)$ \\
$\mathrm{C}(3)-\mathrm{C}(2)-\mathrm{S}(1)$ & $110.22(8)$ \\
$\mathrm{C}(1)-\mathrm{C}(2)-\mathrm{S}(1)$ & $108.64(8)$ \\
$\mathrm{C}(4)-\mathrm{C}(3)-\mathrm{C}(2)$ & $111.59(10)$ \\
$\mathrm{C}(5)-\mathrm{C}(4)-\mathrm{C}(3)$ & $110.00(11)$ \\
& \\
& \\
&
\end{tabular}




$\begin{array}{ll}\mathrm{C}(4)-\mathrm{C}(5)-\mathrm{C}(6) & 109.92(12) \\ \mathrm{C}(5)-\mathrm{C}(6)-\mathrm{C}(1) & 113.23(11) \\ \mathrm{C}(8)-\mathrm{C}(7)-\mathrm{C}(1) & 117.00(10) \\ \mathrm{C}(9)-\mathrm{C}(8)-\mathrm{C}(10) & 121.50(15) \\ \mathrm{C}(9)-\mathrm{C}(8)-\mathrm{C}(7) & 121.26(14) \\ \mathrm{C}(10)-\mathrm{C}(8)-\mathrm{C}(7) & 117.17(13) \\ \mathrm{C}(17)-\mathrm{C}(12)-\mathrm{C}(13) & 119.28(11) \\ \mathrm{C}(17)-\mathrm{C}(12)-\mathrm{S}(1) & 125.57(9) \\ \mathrm{C}(13)-\mathrm{C}(12)-\mathrm{S}(1) & 115.12(9) \\ \mathrm{C}(14)-\mathrm{C}(13)-\mathrm{C}(12) & 120.40(12) \\ \mathrm{C}(13)-\mathrm{C}(14)-\mathrm{C}(15) & 120.21(13) \\ \mathrm{C}(16)-\mathrm{C}(15)-\mathrm{C}(14) & 119.62(12) \\ \mathrm{C}(15)-\mathrm{C}(16)-\mathrm{C}(17) & 120.71(12) \\ \mathrm{C}(12)-\mathrm{C}(17)-\mathrm{C}(16) & 119.78(12)\end{array}$


Table 4. Anisotropic displacement parameters $\left(\AA^{2} \times 10^{3}\right)$ for kaw49. The anisotropic displacement factor exponent takes the form: $-2 \square^{2}\left[\mathrm{~h}^{2} \mathrm{a}^{* 2} \mathrm{U}^{11}+\ldots+2 \mathrm{hk} \mathrm{a}^{*} \mathrm{~b}^{*} \mathrm{U}^{12}\right]$

\begin{tabular}{lcccccc}
\hline & $\mathrm{U}^{11}$ & $\mathrm{U}^{22}$ & $\mathrm{U}^{33}$ & $\mathrm{U}^{23}$ & $\mathrm{U}^{13}$ & $\mathrm{U}$ \\
\hline $\mathrm{S}(1)$ & $39(1)$ & $32(1)$ & $21(1)$ & $1(1)$ & $-5(1)$ & $10(1)$ \\
$\mathrm{O}(1)$ & $25(1)$ & $48(1)$ & $26(1)$ & $-10(1)$ & $-5(1)$ & $4(1)$ \\
$\mathrm{C}(1)$ & $22(1)$ & $32(1)$ & $24(1)$ & $-1(1)$ & $-2(1)$ & $1(1)$ \\
$\mathrm{C}(2)$ & $24(1)$ & $27(1)$ & $22(1)$ & $1(1)$ & $-1(1)$ & $2(1)$ \\
$\mathrm{C}(3)$ & $28(1)$ & $36(1)$ & $28(1)$ & $3(1)$ & $4(1)$ & $-2(1)$ \\
$\mathrm{C}(4)$ & $36(1)$ & $32(1)$ & $42(1)$ & $8(1)$ & $4(1)$ & $-3(1)$ \\
$\mathrm{C}(5)$ & $35(1)$ & $29(1)$ & $48(1)$ & $-2(1)$ & $2(1)$ & $-3(1)$ \\
$\mathrm{C}(6)$ & $27(1)$ & $31(1)$ & $35(1)$ & $-5(1)$ & $2(1)$ & $2(1)$ \\
$\mathrm{C}(7)$ & $31(1)$ & $38(1)$ & $24(1)$ & $4(1)$ & $3(1)$ & $1(1)$ \\
$\mathrm{C}(8)$ & $30(1)$ & $39(1)$ & $31(1)$ & $5(1)$ & $6(1)$ & $-5(1)$ \\
$\mathrm{C}(9)$ & $44(1)$ & $58(1)$ & $50(1)$ & $-13(1)$ & $6(1)$ & $-20(1)$ \\
$\mathrm{C}(10)$ & $30(1)$ & $52(1)$ & $47(1)$ & $1(1)$ & $7(1)$ & $0(1)$ \\
$\mathrm{C}(11)$ & $36(1)$ & $67(1)$ & $32(1)$ & $-19(1)$ & $-8(1)$ & $6(1)$ \\
$\mathrm{C}(12)$ & $24(1)$ & $26(1)$ & $23(1)$ & $2(1)$ & $-1(1)$ & $-3(1)$ \\
$\mathrm{C}(13)$ & $31(1)$ & $29(1)$ & $28(1)$ & $3(1)$ & $-4(1)$ & $3(1)$ \\
$\mathrm{C}(14)$ & $39(1)$ & $28(1)$ & $34(1)$ & $-1(1)$ & $1(1)$ & $4(1)$ \\
$\mathrm{C}(15)$ & $42(1)$ & $37(1)$ & $25(1)$ & $-3(1)$ & $1(1)$ & $-4(1)$ \\
$\mathrm{C}(16)$ & $32(1)$ & $39(1)$ & $24(1)$ & $3(1)$ & $-6(1)$ & $-2(1)$ \\
$\mathrm{C}(17)$ & $27(1)$ & $32(1)$ & $26(1)$ & $2(1)$ & $-4(1)$ & $3(1)$ \\
& & & & & & \\
\hline
\end{tabular}


Table 5. Hydrogen coordinates ( x 104) and isotropic displacement parameters $\left(\AA^{2} \times 10^{3}\right)$ for kaw49.

$\mathrm{X}$

$\mathrm{y} \quad \mathrm{z} \quad \mathrm{U}(\mathrm{eq})$

\begin{tabular}{|c|c|c|c|c|}
\hline $\mathrm{H}(2)$ & $1990(20)$ & $1965(17)$ & 797(7) & $29(4)$ \\
\hline $\mathrm{H}(3 \mathrm{~A})$ & $4448(19)$ & $2624(17)$ & $200(8)$ & $30(4)$ \\
\hline $\mathrm{H}(3 \mathrm{~B})$ & $5320(20)$ & $3095(19)$ & $878(8)$ & $36(4)$ \\
\hline $\mathrm{H}(4 \mathrm{~A})$ & $4260(20)$ & $5154(19)$ & $360(8)$ & $35(4)$ \\
\hline $\mathrm{H}(4 \mathrm{~B})$ & $2440(20)$ & $4488(19)$ & $353(8)$ & $41(4)$ \\
\hline $\mathrm{H}(5 \mathrm{~A})$ & $2710(20)$ & $6111(18)$ & $1238(8)$ & $38(4)$ \\
\hline $\mathrm{H}(5 \mathrm{~B})$ & $4150(20)$ & 5183(19) & $1534(8)$ & $45(5)$ \\
\hline $\mathrm{H}(6 \mathrm{~A})$ & $910(20)$ & $4071(16)$ & $1383(7)$ & $28(4)$ \\
\hline $\mathrm{H}(6 \mathrm{~B})$ & $1660(20)$ & $4482(19)$ & 2077(9) & $43(5)$ \\
\hline $\mathrm{H}(7 \mathrm{~A})$ & $1850(20)$ & $705(19)$ & $2078(8)$ & $38(4)$ \\
\hline $\mathrm{H}(7 \mathrm{~B})$ & $1370(20)$ & 2031(19) & $2528(8)$ & $41(5)$ \\
\hline $\mathrm{H}(9 \mathrm{~A})$ & $110(30)$ & $40(20)$ & $1168(10)$ & $64(6)$ \\
\hline $\mathrm{H}(9 \mathrm{~B})$ & $-1840(30)$ & $610(20)$ & $1116(12)$ & $76(7)$ \\
\hline $\mathrm{H}(10 \mathrm{~A})$ & $-1300(30)$ & $3500(20)$ & 2091(10) & $58(6)$ \\
\hline $\mathrm{H}(10 \mathrm{~B})$ & $-1650(30)$ & $2300(20)$ & $2563(11)$ & $64(6)$ \\
\hline $\mathrm{H}(10 \mathrm{C})$ & $-2640(30)$ & $2330(20)$ & $1927(10)$ & $52(5)$ \\
\hline $\mathrm{H}(11 \mathrm{~A})$ & $5310(20)$ & $3090(20)$ & $2877(9)$ & $46(5)$ \\
\hline $\mathrm{H}(11 \mathrm{~B})$ & $3470(30)$ & $2940(20)$ & $2963(11)$ & $65(6)$ \\
\hline $\mathrm{H}(11 \mathrm{C})$ & $4180(30)$ & $4310(30)$ & $2647(12)$ & $80(8)$ \\
\hline $\mathrm{H}(13)$ & $5710(20)$ & $-1892(19)$ & $566(8)$ & $38(4)$ \\
\hline $\mathrm{H}(14)$ & $5850(30)$ & $-3030(20)$ & $-457(9)$ & $54(5)$ \\
\hline $\mathrm{H}(15)$ & $4100(20)$ & $-2235(19)$ & $-1303(9)$ & $43(4)$ \\
\hline $\mathrm{H}(16)$ & $2450(20)$ & $-287(19)$ & $-1156(9)$ & $43(4)$ \\
\hline $\mathrm{H}(17)$ & $2280(20)$ & $786(17)$ & $-145(7)$ & $26(4)$ \\
\hline
\end{tabular}


Table 6. Torsion angles $\left[{ }^{\circ}\right]$ for kaw49.

\begin{tabular}{lc}
\hline $\mathrm{C}(11)-\mathrm{O}(1)-\mathrm{C}(1)-\mathrm{C}(6)$ & $52.12(16)$ \\
$\mathrm{C}(11)-\mathrm{O}(1)-\mathrm{C}(1)-\mathrm{C}(7)$ & $-71.63(16)$ \\
$\mathrm{C}(11)-\mathrm{O}(1)-\mathrm{C}(1)-\mathrm{C}(2)$ & $168.78(13)$ \\
$\mathrm{O}(1)-\mathrm{C}(1)-\mathrm{C}(2)-\mathrm{C}(3)$ & $-66.15(12)$ \\
$\mathrm{C}(6)-\mathrm{C}(1)-\mathrm{C}(2)-\mathrm{C}(3)$ & $53.14(12)$ \\
$\mathrm{C}(7)-\mathrm{C}(1)-\mathrm{C}(2)-\mathrm{C}(3)$ & $176.94(10)$ \\
$\mathrm{O}(1)-\mathrm{C}(1)-\mathrm{C}(2)-\mathrm{S}(1)$ & $56.08(11)$ \\
$\mathrm{C}(6)-\mathrm{C}(1)-\mathrm{C}(2)-\mathrm{S}(1)$ & $175.38(8)$ \\
$\mathrm{C}(7)-\mathrm{C}(1)-\mathrm{C}(2)-\mathrm{S}(1)$ & $-60.82(11)$ \\
$\mathrm{C}(12)-\mathrm{S}(1)-\mathrm{C}(2)-\mathrm{C}(3)$ & $-70.32(9)$ \\
$\mathrm{C}(12)-\mathrm{S}(1)-\mathrm{C}(2)-\mathrm{C}(1)$ & $166.14(8)$ \\
$\mathrm{C}(1)-\mathrm{C}(2)-\mathrm{C}(3)-\mathrm{C}(4)$ & $-55.72(14)$ \\
$\mathrm{S}(1)-\mathrm{C}(2)-\mathrm{C}(3)-\mathrm{C}(4)$ & $-177.06(9)$ \\
$\mathrm{C}(2)-\mathrm{C}(3)-\mathrm{C}(4)-\mathrm{C}(5)$ & $57.03(15)$ \\
$\mathrm{C}(3)-\mathrm{C}(4)-\mathrm{C}(5)-\mathrm{C}(6)$ & $-57.62(15)$ \\
$\mathrm{C}(4)-\mathrm{C}(5)-\mathrm{C}(6)-\mathrm{C}(1)$ & $58.61(15)$ \\
$\mathrm{O}(1)-\mathrm{C}(1)-\mathrm{C}(6)-\mathrm{C}(5)$ & $58.38(14)$ \\
$\mathrm{C}(7)-\mathrm{C}(1)-\mathrm{C}(6)-\mathrm{C}(5)$ & $-179.55(11)$ \\
$\mathrm{C}(2)-\mathrm{C}(1)-\mathrm{C}(6)-\mathrm{C}(5)$ & $-55.21(14)$ \\
$\mathrm{O}(1)-\mathrm{C}(1)-\mathrm{C}(7)-\mathrm{C}(8)$ & $178.30(11)$ \\
$\mathrm{C}(6)-\mathrm{C}(1)-\mathrm{C}(7)-\mathrm{C}(8)$ & $54.15(15)$ \\
$\mathrm{C}(2)-\mathrm{C}(1)-\mathrm{C}(7)-\mathrm{C}(8)$ & $-67.92(15)$ \\
$\mathrm{C}(1)-\mathrm{C}(7)-\mathrm{C}(8)-\mathrm{C}(9)$ & $85.46(18)$ \\
$\mathrm{C}(1)-\mathrm{C}(7)-\mathrm{C}(8)-\mathrm{C}(10)$ & $-97.55(14)$ \\
$\mathrm{C}(2)-\mathrm{S}(1)-\mathrm{C}(12)-\mathrm{C}(17)$ & $-17.73(12)$ \\
$\mathrm{C}(2)-\mathrm{S}(1)-\mathrm{C}(12)-\mathrm{C}(13)$ & $164.19(9)$ \\
$\mathrm{C}(17)-\mathrm{C}(12)-\mathrm{C}(13)-\mathrm{C}(14)$ & $-0.59(19)$ \\
$\mathrm{S}(1)-\mathrm{C}(12)-\mathrm{C}(13)-\mathrm{C}(14)$ & $177.62(10)$ \\
$\mathrm{C}(12)-\mathrm{C}(13)-\mathrm{C}(14)-\mathrm{C}(15)$ & $-0.2(2)$ \\
$\mathrm{C}(13)-\mathrm{C}(14)-\mathrm{C}(15)-\mathrm{C}(16)$ & $0.9(2)$ \\
$\mathrm{C}(14)-\mathrm{C}(15)-\mathrm{C}(16)-\mathrm{C}(17)$ & $-0.8(2)$ \\
$\mathrm{C}(13)-\mathrm{C}(12)-\mathrm{C}(17)-\mathrm{C}(16)$ & $0.67(18)$ \\
$\mathrm{S}(1)-\mathrm{C}(12)-\mathrm{C}(17)-\mathrm{C}(16)$ & $-177.34(10)$ \\
$\mathrm{C}(15)-\mathrm{C}(16)-\mathrm{C}(17)-\mathrm{C}(12)$ & \\
&
\end{tabular}


Table 1. Crystal data and structure refinement for Nitrile $\mathbf{1 0 .}$

Identification code

Empirical formula

Formula weight

Temperature

Wavelength

Crystal system

Space group

Unit cell dimensions

Volume

Z

Density (calculated)

Absorption coefficient

$\mathrm{F}(000)$

Crystal size

Theta range for data collection

Index ranges

Reflections collected

Independent reflections

Completeness to theta $=28.31^{\circ}$

Absorption correction

Max. and min. transmission

Refinement method

Data / restraints / parameters

Goodness-of-fit on $\mathrm{F}^{2}$

Final $\mathrm{R}$ indices [I $>2 \operatorname{sigma}(\mathrm{I})]$

$\mathrm{R}$ indices (all data)

Extinction coefficient

Largest diff. peak and hole
Nitrile 10

$\mathrm{C}_{14} \mathrm{H}_{17} \mathrm{~N} \mathrm{O} \mathrm{S}$

247.35

163(2) K

$0.71073 \AA$

Orthorhombic

Pbca

$\mathrm{a}=8.2284(5) \AA$

$\square=90^{\circ}$.

$\mathrm{b}=14.6764(9) \AA$

$\square=90^{\circ}$.

$c=21.4924(13) \AA$

$\square=90^{\circ}$.
$\mathrm{MeO} \subseteq \mathrm{CN}$<smiles>CC1(C)CCCC[C@H]1[SnH2]</smiles>

10

2595.5(3) $\AA^{3}$

8

$1.266 \mathrm{Mg} / \mathrm{m}^{3}$

$0.233 \mathrm{~mm}^{-1}$

1056

$0.37 \times 0.35 \times 0.25 \mathrm{~mm}^{3}$

1.90 to $28.31^{\circ}$.

$-10 h \quad 10,-19 k \quad k \quad 19,-28 \quad l \quad 28$

25543

3186 [R(int) $=0.0262]$

$98.6 \%$

Semi-empirical from equivalents

0.9441 and 0.9188

Full-matrix least-squares on $\mathrm{F}^{2}$

3186 / 0 / 223

1.048

$\mathrm{R} 1=0.0308, w \mathrm{R} 2=0.0800$

$\mathrm{R} 1=0.0371, \mathrm{wR} 2=0.0851$

$0.0014(6)$

0.318 and -0.275 e. $\AA^{-3}$ 
Table 2. Atomic coordinates ( $\left.\mathrm{x} 10^{4}\right)$ and equivalent isotropic displacement parameters $\left(\AA^{2} \times 10^{3}\right)$ for kaw44. $U(\mathrm{eq})$ is defined as one third of the trace of the orthogonalized $U^{\mathrm{ij}}$ tensor.

\begin{tabular}{lrrrr}
\hline & $\mathrm{x}$ & $\mathrm{y}$ & $\mathrm{z}$ & $\mathrm{U}(\mathrm{eq})$ \\
\hline $\mathrm{S}(1)$ & $-33(1)$ & $5264(1)$ & $1801(1)$ & $28(1)$ \\
$\mathrm{O}(1)$ & $1513(1)$ & $6849(1)$ & $1229(1)$ & $24(1)$ \\
$\mathrm{N}(1)$ & $-1252(1)$ & $6103(1)$ & $177(1)$ & $34(1)$ \\
$\mathrm{C}(1)$ & $1495(1)$ & $6100(1)$ & $812(1)$ & $19(1)$ \\
$\mathrm{C}(2)$ & $1622(1)$ & $5249(1)$ & $1228(1)$ & $20(1)$ \\
$\mathrm{C}(3)$ & $3284(2)$ & $5212(1)$ & $1543(1)$ & $28(1)$ \\
$\mathrm{C}(4)$ & $4667(2)$ & $5236(1)$ & $1069(1)$ & $32(1)$ \\
$\mathrm{C}(5)$ & $4537(1)$ & $6072(1)$ & $651(1)$ & $29(1)$ \\
$\mathrm{C}(6)$ & $2883(1)$ & $6106(1)$ & $332(1)$ & $23(1)$ \\
$\mathrm{C}(7)$ & $1548(2)$ & $7734(1)$ & $954(1)$ & $35(1)$ \\
$\mathrm{C}(8)$ & $-81(1)$ & $6092(1)$ & $466(1)$ & $23(1)$ \\
$\mathrm{C}(9)$ & $-516(1)$ & $4083(1)$ & $1835(1)$ & $21(1)$ \\
$\mathrm{C}(10)$ & $100(1)$ & $3543(1)$ & $2313(1)$ & $24(1)$ \\
$\mathrm{C}(11)$ & $-368(2)$ & $2635(1)$ & $2361(1)$ & $28(1)$ \\
$\mathrm{C}(12)$ & $-1433(2)$ & $2263(1)$ & $1935(1)$ & $31(1)$ \\
$\mathrm{C}(13)$ & $-2023(2)$ & $2794(1)$ & $1451(1)$ & $33(1)$ \\
$\mathrm{C}(14)$ & $-1576(1)$ & $3702(1)$ & $1401(1)$ & $28(1)$ \\
& & & & \\
\hline
\end{tabular}


Table 3. Bond lengths $[\AA ̊ \mathrm{~A}]$ and angles $\left[{ }^{\circ}\right]$ for kaw44.

\begin{tabular}{|c|c|}
\hline$S(1)-C(9)$ & $1.7809(11)$ \\
\hline $\mathrm{S}(1)-\mathrm{C}(2)$ & $1.8357(11)$ \\
\hline $\mathrm{O}(1)-\mathrm{C}(1)$ & $1.4178(12)$ \\
\hline $\mathrm{O}(1)-\mathrm{C}(7)$ & $1.4264(14)$ \\
\hline $\mathrm{N}(1)-\mathrm{C}(8)$ & $1.1461(15)$ \\
\hline $\mathrm{C}(1)-\mathrm{C}(8)$ & $1.4958(14)$ \\
\hline$C(1)-C(2)$ & $1.5391(14)$ \\
\hline$C(1)-C(6)$ & $1.5395(14)$ \\
\hline $\mathrm{C}(2)-\mathrm{C}(3)$ & $1.5270(16)$ \\
\hline$C(3)-C(4)$ & $1.5273(18)$ \\
\hline$C(4)-C(5)$ & $1.5247(18)$ \\
\hline$C(5)-C(6)$ & $1.5247(16)$ \\
\hline $\mathrm{C}(9)-\mathrm{C}(14)$ & $1.3927(16)$ \\
\hline $\mathrm{C}(9)-\mathrm{C}(10)$ & $1.3936(15)$ \\
\hline $\mathrm{C}(10)-\mathrm{C}(11)$ & $1.3921(16)$ \\
\hline $\mathrm{C}(11)-\mathrm{C}(12)$ & $1.3810(18)$ \\
\hline $\mathrm{C}(12)-\mathrm{C}(13)$ & $1.3877(19)$ \\
\hline $\mathrm{C}(13)-\mathrm{C}(14)$ & $1.3866(18)$ \\
\hline $\mathrm{C}(9)-\mathrm{S}(1)-\mathrm{C}(2)$ & $100.43(5)$ \\
\hline $\mathrm{C}(1)-\mathrm{O}(1)-\mathrm{C}(7)$ & $116.41(9)$ \\
\hline $\mathrm{O}(1)-\mathrm{C}(1)-\mathrm{C}(8)$ & $109.22(8)$ \\
\hline $\mathrm{O}(1)-\mathrm{C}(1)-\mathrm{C}(2)$ & $105.22(8)$ \\
\hline $\mathrm{C}(8)-\mathrm{C}(1)-\mathrm{C}(2)$ & $109.98(8)$ \\
\hline $\mathrm{O}(1)-\mathrm{C}(1)-\mathrm{C}(6)$ & $114.29(8)$ \\
\hline $\mathrm{C}(8)-\mathrm{C}(1)-\mathrm{C}(6)$ & $107.99(8)$ \\
\hline $\mathrm{C}(2)-\mathrm{C}(1)-\mathrm{C}(6)$ & $110.09(8)$ \\
\hline $\mathrm{C}(3)-\mathrm{C}(2)-\mathrm{C}(1)$ & $110.27(9)$ \\
\hline$C(3)-C(2)-S(1)$ & $111.57(8)$ \\
\hline$C(1)-C(2)-S(1)$ & $109.21(7)$ \\
\hline $\mathrm{C}(2)-\mathrm{C}(3)-\mathrm{C}(4)$ & $111.77(9)$ \\
\hline$C(5)-C(4)-C(3)$ & $111.04(10)$ \\
\hline$C(6)-C(5)-C(4)$ & $110.72(10)$ \\
\hline$C(5)-C(6)-C(1)$ & 111.11(9) \\
\hline
\end{tabular}




$\begin{array}{ll}\mathrm{N}(1)-\mathrm{C}(8)-\mathrm{C}(1) & 176.88(12) \\ \mathrm{C}(14)-\mathrm{C}(9)-\mathrm{C}(10) & 119.60(10) \\ \mathrm{C}(14)-\mathrm{C}(9)-\mathrm{S}(1) & 120.19(9) \\ \mathrm{C}(10)-\mathrm{C}(9)-\mathrm{S}(1) & 120.13(9) \\ \mathrm{C}(11)-\mathrm{C}(10)-\mathrm{C}(9) & 119.89(11) \\ \mathrm{C}(12)-\mathrm{C}(11)-\mathrm{C}(10) & 120.30(11) \\ \mathrm{C}(11)-\mathrm{C}(12)-\mathrm{C}(13) & 119.87(11) \\ \mathrm{C}(14)-\mathrm{C}(13)-\mathrm{C}(12) & 120.30(12) \\ \mathrm{C}(13)-\mathrm{C}(14)-\mathrm{C}(9) & 120.02(11)\end{array}$


Table 4. Anisotropic displacement parameters $\left(\AA^{2} \times 10^{3}\right)$ for kaw44. The anisotropic displacement factor exponent takes the form: $-2 \square^{2}\left[h^{2} a^{* 2} U^{11}+\ldots+2 h k a^{*} b^{*} U^{12}\right]$

\begin{tabular}{lllllll}
\hline & $\mathrm{U}^{11}$ & $\mathrm{U}^{22}$ & $\mathrm{U}^{33}$ & $\mathrm{U}^{23}$ & $\mathrm{U}^{13}$ & $\mathrm{U}^{12}$ \\
\hline $\mathrm{S}(1)$ & $43(1)$ & $17(1)$ & $25(1)$ & $1(1)$ & $15(1)$ & $3(1)$ \\
$\mathrm{O}(1)$ & $36(1)$ & $14(1)$ & $20(1)$ & $-1(1)$ & $1(1)$ & $-1(1)$ \\
$\mathrm{N}(1)$ & $27(1)$ & $40(1)$ & $36(1)$ & $0(1)$ & $-5(1)$ & $4(1)$ \\
$\mathrm{C}(1)$ & $22(1)$ & $18(1)$ & $17(1)$ & $0(1)$ & $-1(1)$ & $0(1)$ \\
$\mathrm{C}(2)$ & $26(1)$ & $17(1)$ & $16(1)$ & $0(1)$ & $3(1)$ & $2(1)$ \\
$\mathrm{C}(3)$ & $34(1)$ & $25(1)$ & $23(1)$ & $1(1)$ & $-7(1)$ & $6(1)$ \\
$\mathrm{C}(4)$ & $23(1)$ & $33(1)$ & $39(1)$ & $-4(1)$ & $-7(1)$ & $6(1)$ \\
$\mathrm{C}(5)$ & $21(1)$ & $34(1)$ & $33(1)$ & $-3(1)$ & $0(1)$ & $-4(1)$ \\
$\mathrm{C}(6)$ & $23(1)$ & $26(1)$ & $19(1)$ & $0(1)$ & $2(1)$ & $-3(1)$ \\
$\mathrm{C}(7)$ & $53(1)$ & $17(1)$ & $36(1)$ & $3(1)$ & $5(1)$ & $-2(1)$ \\
$\mathrm{C}(8)$ & $24(1)$ & $22(1)$ & $22(1)$ & $2(1)$ & $2(1)$ & $1(1)$ \\
$\mathrm{C}(9)$ & $25(1)$ & $19(1)$ & $18(1)$ & $1(1)$ & $6(1)$ & $3(1)$ \\
$\mathrm{C}(10)$ & $27(1)$ & $23(1)$ & $20(1)$ & $3(1)$ & $0(1)$ & $-1(1)$ \\
$\mathrm{C}(11)$ & $34(1)$ & $23(1)$ & $28(1)$ & $6(1)$ & $6(1)$ & $2(1)$ \\
$\mathrm{C}(12)$ & $31(1)$ & $22(1)$ & $41(1)$ & $-5(1)$ & $12(1)$ & $-4(1)$ \\
$\mathrm{C}(13)$ & $27(1)$ & $38(1)$ & $36(1)$ & $-13(1)$ & $-2(1)$ & $-2(1)$ \\
$\mathrm{C}(14)$ & $29(1)$ & $33(1)$ & $22(1)$ & $-2(1)$ & $-2(1)$ & $7(1)$ \\
& & & & & & \\
\hline
\end{tabular}


Table 5. Hydrogen coordinates ( $\left.\mathrm{x} 10^{4}\right)$ and isotropic displacement parameters $\left(\AA^{2} \mathrm{x} 10^{3}\right)$ for kaw44.

\begin{tabular}{|c|c|c|c|c|}
\hline & $\mathrm{x}$ & $\mathrm{y}$ & $\mathrm{z}$ & $\mathrm{U}(\mathrm{eq})$ \\
\hline $\mathrm{H}(2)$ & $1455(16)$ & $4706(9)$ & $972(6)$ & $25(3)$ \\
\hline $\mathrm{H}(3 \mathrm{~A})$ & $3314(18)$ & $4646(10)$ & $1790(7)$ & $34(4)$ \\
\hline $\mathrm{H}(3 \mathrm{~B})$ & $3365(18)$ & $5728(11)$ & $1824(7)$ & $35(4)$ \\
\hline $\mathrm{H}(4 \mathrm{~A})$ & $4606(19)$ & $4658(11)$ & $819(8)$ & $40(4)$ \\
\hline $\mathrm{H}(4 \mathrm{~B})$ & $5710(20)$ & $5243(11)$ & $1286(8)$ & $45(4)$ \\
\hline $\mathrm{H}(5 \mathrm{~A})$ & $4675(18)$ & $6621(10)$ & $899(7)$ & $34(4)$ \\
\hline $\mathrm{H}(5 \mathrm{~B})$ & $5380(20)$ & $6060(10)$ & $337(7)$ & $40(4)$ \\
\hline $\mathrm{H}(6 \mathrm{~A})$ & $2721(16)$ & $5584(9)$ & $76(6)$ & $25(3)$ \\
\hline $\mathrm{H}(6 \mathrm{~B})$ & $2736(17)$ & $6643(9)$ & $66(6)$ & $28(3)$ \\
\hline $\mathrm{H}(7 \mathrm{~A})$ & $1330(20)$ & $8160(11)$ & 1293(8) & $43(4)$ \\
\hline $\mathrm{H}(7 \mathrm{~B})$ & $720(20)$ & $7799(11)$ & $629(8)$ & $49(4)$ \\
\hline $\mathrm{H}(7 \mathrm{C})$ & $2590(20)$ & $7852(11)$ & $779(8)$ & $47(4)$ \\
\hline $\mathrm{H}(10)$ & $827(17)$ & $3807(9)$ & $2614(7)$ & $30(4)$ \\
\hline $\mathrm{H}(11)$ & $70(20)$ & $2289(13)$ & 2704(8) & $51(5)$ \\
\hline $\mathrm{H}(12)$ & $-1770(20)$ & 1651(11) & $1974(7)$ & $41(4)$ \\
\hline $\mathrm{H}(13)$ & $-2730(20)$ & $2537(12)$ & $1158(8)$ & $51(5)$ \\
\hline $\mathrm{H}(14)$ & $-1976(18)$ & $4084(10)$ & $1074(7)$ & $37(4)$ \\
\hline
\end{tabular}


Table 6. Torsion angles $\left[{ }^{\circ}\right]$ for kaw 44.

\begin{tabular}{lc}
\hline $\mathrm{C}(7)-\mathrm{O}(1)-\mathrm{C}(1)-\mathrm{C}(8)$ & $-67.17(12)$ \\
$\mathrm{C}(7)-\mathrm{O}(1)-\mathrm{C}(1)-\mathrm{C}(2)$ & $174.80(10)$ \\
$\mathrm{C}(7)-\mathrm{O}(1)-\mathrm{C}(1)-\mathrm{C}(6)$ & $53.91(13)$ \\
$\mathrm{O}(1)-\mathrm{C}(1)-\mathrm{C}(2)-\mathrm{C}(3)$ & $-67.08(10)$ \\
$\mathrm{C}(8)-\mathrm{C}(1)-\mathrm{C}(2)-\mathrm{C}(3)$ & $175.40(9)$ \\
$\mathrm{C}(6)-\mathrm{C}(1)-\mathrm{C}(2)-\mathrm{C}(3)$ & $56.53(11)$ \\
$\mathrm{O}(1)-\mathrm{C}(1)-\mathrm{C}(2)-\mathrm{S}(1)$ & $55.85(9)$ \\
$\mathrm{C}(8)-\mathrm{C}(1)-\mathrm{C}(2)-\mathrm{S}(1)$ & $-61.66(10)$ \\
$\mathrm{C}(6)-\mathrm{C}(1)-\mathrm{C}(2)-\mathrm{S}(1)$ & $179.46(7)$ \\
$\mathrm{C}(9)-\mathrm{S}(1)-\mathrm{C}(2)-\mathrm{C}(3)$ & $-95.09(8)$ \\
$\mathrm{C}(9)-\mathrm{S}(1)-\mathrm{C}(2)-\mathrm{C}(1)$ & $142.75(7)$ \\
$\mathrm{C}(1)-\mathrm{C}(2)-\mathrm{C}(3)-\mathrm{C}(4)$ & $-56.30(12)$ \\
$\mathrm{S}(1)-\mathrm{C}(2)-\mathrm{C}(3)-\mathrm{C}(4)$ & $-177.84(8)$ \\
$\mathrm{C}(2)-\mathrm{C}(3)-\mathrm{C}(4)-\mathrm{C}(5)$ & $55.94(13)$ \\
$\mathrm{C}(3)-\mathrm{C}(4)-\mathrm{C}(5)-\mathrm{C}(6)$ & $-55.75(14)$ \\
$\mathrm{C}(4)-\mathrm{C}(5)-\mathrm{C}(6)-\mathrm{C}(1)$ & $57.00(13)$ \\
$\mathrm{O}(1)-\mathrm{C}(1)-\mathrm{C}(6)-\mathrm{C}(5)$ & $60.73(12)$ \\
$\mathrm{C}(8)-\mathrm{C}(1)-\mathrm{C}(6)-\mathrm{C}(5)$ & $-177.52(9)$ \\
$\mathrm{C}(2)-\mathrm{C}(1)-\mathrm{C}(6)-\mathrm{C}(5)$ & $-57.43(12)$ \\
$\mathrm{O}(1)-\mathrm{C}(1)-\mathrm{C}(8)-\mathrm{N}(1)$ & $102(2)$ \\
$\mathrm{C}(2)-\mathrm{C}(1)-\mathrm{C}(8)-\mathrm{N}(1)$ & $-143(2)$ \\
$\mathrm{C}(6)-\mathrm{C}(1)-\mathrm{C}(8)-\mathrm{N}(1)$ & $-23(2)$ \\
$\mathrm{C}(2)-\mathrm{S}(1)-\mathrm{C}(9)-\mathrm{C}(14)$ & $-84.56(10)$ \\
$\mathrm{C}(2)-\mathrm{S}(1)-\mathrm{C}(9)-\mathrm{C}(10)$ & $98.63(18)$ \\
$\mathrm{C}(14)-\mathrm{C}(9)-\mathrm{C}(10)-\mathrm{C}(11)$ & $-1.06(16)$ \\
$\mathrm{S}(1)-\mathrm{C}(9)-\mathrm{C}(10)-\mathrm{C}(11)$ & $175.77(9)$ \\
$\mathrm{C}(9)-\mathrm{C}(10)-\mathrm{C}(11)-\mathrm{C}(12)$ & $0.30(17)$ \\
$\mathrm{C}(10)-\mathrm{C}(11)-\mathrm{C}(12)-\mathrm{C}(13)$ & \\
$\mathrm{C}(11)-\mathrm{C}(12)-\mathrm{C}(13)-\mathrm{C}(14)$ & \\
$\mathrm{C}(12)-\mathrm{C}(13)-\mathrm{C}(14)-\mathrm{C}(9)$ & \\
$\mathrm{C}(10)-\mathrm{C}(9)-\mathrm{C}(14)-\mathrm{C}(13)$ & \\
$\mathrm{S}(1)-\mathrm{C}(9)-\mathrm{C}(14)-\mathrm{C}(13)$ & \\
\hline & \\
& \\
& \\
&
\end{tabular}


Table 1. Crystal data and structure refinement for Nitrile S-11.

Identification code

Empirical formula

Formula weight

Temperature

Wavelength

Crystal system

Space group

Unit cell dimensions

Volume

Z

Density (calculated)

Absorption coefficient

$\mathrm{F}(000)$

Crystal color

Crystal size
Nitrile S-11

$\mathrm{C}_{18} \mathrm{H}_{27} \mathrm{~N} \mathrm{O}_{2} \mathrm{~S}$

321.47

148(2) K

$0.71073 \AA$

Monoclinic

$P 2{ }_{1} / c$

$\mathrm{a}=8.738(9) \AA$

$\square=90^{\circ}$.

$\mathrm{b}=30.34(3) \AA$

$\square=91.031(16)^{\circ}$.

$c=13.872(14) \AA$

$\square=90^{\circ}$.

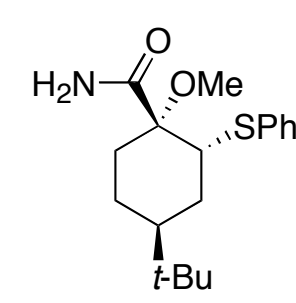

S-11
3677(6) $\AA^{3}$

8

$1.161 \mathrm{Mg} / \mathrm{m}^{3}$

$0.183 \mathrm{~mm}^{-1}$

1392

colorless

$0.39 \times 0.22 \times 0.07 \mathrm{~mm}^{3}$ 
Table 1. Crystal data and structure refinement for Fluorocyclohexanol 18.

Identification code

Empirical formula

Formula weight

Temperature

Wavelength

Crystal system

Space group

Unit cell dimensions

Volume

Z

Density (calculated)

Absorption coefficient

$\mathrm{F}(000)$

Crystal color

Crystal size

Theta range for data collection

Index ranges

Reflections collected

Independent reflections

Completeness to theta $=27.10^{\circ}$

Absorption correction

Max. and min. transmission

Refinement method

Data / restraints / parameters

Goodness-of-fit on $\mathrm{F}^{2}$

Final $R$ indices $[I>2 \operatorname{sigma}(I)=3726$ data $]$

$\mathrm{R}$ indices (all data; 0.78))

Absolute structure parameter

Extinction coefficient

Largest diff. peak and hole
Flurocyclohexanol 18

$\mathrm{C}_{12} \mathrm{H}_{15}$ F O

194.24

163(2) K

$0.71073 \AA$

Orthorhombic

Pca $2_{1}$

$\mathrm{a}=14.3874(14) \AA \quad \square=90^{\circ}$.

$\mathrm{b}=5.6698(6) \AA \quad \square=90^{\circ}$.

$\mathrm{c}=24.607(3) \AA \quad \square=90^{\circ}$.

2007.3(4) $\AA^{3}$

8

$1.285 \mathrm{Mg} / \mathrm{m}^{3}$

$0.092 \mathrm{~mm}^{-1}$

832

colorless

$0.33 \times 0.24 \times 0.10 \mathrm{~mm}^{3}$

1.66 to $27.10^{\circ}$.

$-18 h \quad 18,-7 \quad k \quad 7,-31 \quad l \quad 31$

18816

$4403[R($ int $)=0.0426]$

$99.5 \%$

Semi-empirical from equivalents

0.9908 and 0.9702

Full-matrix least-squares on $\mathrm{F}^{2}$

4403 / 3 / 263

1.046

$\mathrm{R} 1=0.0503, \mathrm{wR} 2=0.1352$

$\mathrm{R} 1=0.0635, \mathrm{wR} 2=0.1454$

$0.7(10)$

$0.0013(10)$

0.553 and -0.231 e. $\AA^{-3}$

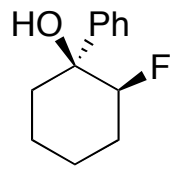

18 
Table 2. Atomic coordinates $\left(\times 10^{4}\right)$ and equivalent isotropic displacement parameters $\left(\AA^{2} \times 10^{3}\right)$ for kaw74. $U(e q)$ is defined as one third of the trace of the orthogonalized $U^{i j}$ tensor.

\begin{tabular}{|c|c|c|c|c|}
\hline & $\mathrm{x}$ & $\mathrm{y}$ & $\mathrm{z}$ & $\mathrm{U}(\mathrm{eq})$ \\
\hline $\mathrm{C}(1)$ & $602(2)$ & $3274(4)$ & $-92(1)$ & $20(1)$ \\
\hline$C(2)$ & $593(2)$ & $5156(5)$ & $344(1)$ & $22(1)$ \\
\hline$C(3)$ & $-189(2)$ & 4901(5) & $748(1)$ & $23(1)$ \\
\hline$C(4)$ & $-1122(2)$ & 4960(5) & $448(1)$ & $28(1)$ \\
\hline$C(5)$ & $-1158(2)$ & $3035(5)$ & $17(1)$ & $29(1)$ \\
\hline$C(6)$ & $-349(2)$ & $3265(5)$ & $-382(1)$ & $26(1)$ \\
\hline$C(7)$ & $1387(2)$ & $3720(5)$ & $-498(1)$ & $21(1)$ \\
\hline$C(8)$ & $1412(2)$ & $5784(5)$ & $-803(1)$ & $24(1)$ \\
\hline$C(9)$ & $2109(2)$ & $6180(5)$ & $-1182(1)$ & $29(1)$ \\
\hline$C(10)$ & 2801(2) & 4518(5) & $-1259(1)$ & $30(1)$ \\
\hline $\mathrm{C}(11)$ & 2792(2) & $2473(5)$ & $-956(1)$ & $29(1)$ \\
\hline$C(12)$ & $2088(2)$ & 2083(5) & $-577(1)$ & $24(1)$ \\
\hline $\mathrm{O}(1)$ & $688(1)$ & 1001(3) & $151(1)$ & $27(1)$ \\
\hline $\mathrm{F}(1)$ & $1443(1)$ & $5019(3)$ & $632(1)$ & $34(1)$ \\
\hline$C(13)$ & $1855(2)$ & $-1840(4)$ & 2023(1) & $17(1)$ \\
\hline$C(14)$ & $1805(2)$ & $180(4)$ & 1611(1) & $20(1)$ \\
\hline$C(15)$ & $2607(2)$ & $204(5)$ & $1215(1)$ & $25(1)$ \\
\hline$C(16)$ & $3526(2)$ & $346(5)$ & $1518(1)$ & $28(1)$ \\
\hline$C(17)$ & $3622(2)$ & $-1668(5)$ & 1928(1) & $25(1)$ \\
\hline$C(18)$ & 2794(2) & $-1693(5)$ & $2322(1)$ & $22(1)$ \\
\hline$C(19)$ & $1048(2)$ & $-1702(4)$ & $2427(1)$ & $18(1)$ \\
\hline$C(20)$ & $957(2)$ & $231(5)$ & $2769(1)$ & $24(1)$ \\
\hline $\mathrm{C}(21)$ & $226(2)$ & $344(6)$ & $3141(1)$ & $30(1)$ \\
\hline $\mathrm{C}(22)$ & $-414(2)$ & $-1465(5)$ & $3172(1)$ & $30(1)$ \\
\hline $\mathrm{C}(23)$ & $-337(2)$ & $-3380(6)$ & $2830(1)$ & $31(1)$ \\
\hline$C(24)$ & $392(2)$ & $-3504(5)$ & $2456(1)$ & $24(1)$ \\
\hline $\mathrm{O}(2)$ & 1861(1) & $-4047(3)$ & $1744(1)$ & $22(1)$ \\
\hline $\mathrm{F}(2)$ & $979(1)$ & $-106(3)$ & $1312(1)$ & $32(1)$ \\
\hline
\end{tabular}


Table 3. Bond lengths $[\AA]$ and angles $\left[{ }^{\circ}\right]$ for kaw74.

\begin{tabular}{|c|c|}
\hline $\mathrm{C}(1)-\mathrm{O}(1)$ & $1.427(3)$ \\
\hline $\mathrm{C}(1)-\mathrm{C}(2)$ & $1.514(4)$ \\
\hline$C(1)-C(7)$ & $1.529(3)$ \\
\hline$C(1)-C(6)$ & $1.542(3)$ \\
\hline $\mathrm{C}(2)-\mathrm{F}(1)$ & $1.416(3)$ \\
\hline$C(2)-C(3)$ & $1.506(4)$ \\
\hline$C(3)-C(4)$ & $1.532(4)$ \\
\hline$C(4)-C(5)$ & $1.522(4)$ \\
\hline$C(5)-C(6)$ & $1.529(4)$ \\
\hline $\mathrm{C}(7)-\mathrm{C}(12)$ & $1.385(4)$ \\
\hline$C(7)-C(8)$ & $1.390(4)$ \\
\hline $\mathrm{C}(8)-\mathrm{C}(9)$ & $1.389(4)$ \\
\hline $\mathrm{C}(9)-\mathrm{C}(10)$ & $1.384(4)$ \\
\hline$C(10)-C(11)$ & $1.379(4)$ \\
\hline $\mathrm{C}(11)-\mathrm{C}(12)$ & $1.395(4)$ \\
\hline $\mathrm{C}(13)-\mathrm{O}(2)$ & $1.427(3)$ \\
\hline$C(13)-C(19)$ & $1.530(3)$ \\
\hline$C(13)-C(14)$ & $1.531(3)$ \\
\hline $\mathrm{C}(13)-\mathrm{C}(18)$ & $1.542(3)$ \\
\hline$C(14)-F(2)$ & $1.407(3)$ \\
\hline$C(14)-C(15)$ & $1.510(3)$ \\
\hline$C(15)-C(16)$ & $1.521(4)$ \\
\hline$C(16)-C(17)$ & $1.530(4)$ \\
\hline $\mathrm{C}(17)-\mathrm{C}(18)$ & $1.535(3)$ \\
\hline$C(19)-C(20)$ & $1.388(4)$ \\
\hline $\mathrm{C}(19)-\mathrm{C}(24)$ & $1.394(3)$ \\
\hline$C(20)-C(21)$ & $1.397(4)$ \\
\hline$C(21)-C(22)$ & $1.379(4)$ \\
\hline$C(22)-C(23)$ & $1.378(4)$ \\
\hline$C(23)-C(24)$ & $1.396(4)$ \\
\hline $\mathrm{O}(1)-\mathrm{C}(1)-\mathrm{C}(2)$ & $109.8(2)$ \\
\hline $\mathrm{O}(1)-\mathrm{C}(1)-\mathrm{C}(7)$ & $111.09(19)$ \\
\hline$C(2)-C(1)-C(7)$ & $110.7(2)$ \\
\hline
\end{tabular}




\begin{tabular}{|c|c|}
\hline $\mathrm{O}(1)-\mathrm{C}(1)-\mathrm{C}(6)$ & $105.6(2)$ \\
\hline$C(2)-C(1)-C(6)$ & $108.9(2)$ \\
\hline $\mathrm{C}(7)-\mathrm{C}(1)-\mathrm{C}(6)$ & $110.7(2)$ \\
\hline $\mathrm{F}(1)-\mathrm{C}(2)-\mathrm{C}(3)$ & $108.1(2)$ \\
\hline $\mathrm{F}(1)-\mathrm{C}(2)-\mathrm{C}(1)$ & $108.0(2)$ \\
\hline $\mathrm{C}(3)-\mathrm{C}(2)-\mathrm{C}(1)$ & $114.0(2)$ \\
\hline $\mathrm{C}(2)-\mathrm{C}(3)-\mathrm{C}(4)$ & $109.5(2)$ \\
\hline$C(5)-C(4)-C(3)$ & $110.5(2)$ \\
\hline $\mathrm{C}(4)-\mathrm{C}(5)-\mathrm{C}(6)$ & $111.1(2)$ \\
\hline $\mathrm{C}(5)-\mathrm{C}(6)-\mathrm{C}(1)$ & $112.2(2)$ \\
\hline$C(12)-C(7)-C(8)$ & $118.1(2)$ \\
\hline $\mathrm{C}(12)-\mathrm{C}(7)-\mathrm{C}(1)$ & $121.2(2)$ \\
\hline $\mathrm{C}(8)-\mathrm{C}(7)-\mathrm{C}(1)$ & $120.7(2)$ \\
\hline $\mathrm{C}(9)-\mathrm{C}(8)-\mathrm{C}(7)$ & $121.2(3)$ \\
\hline $\mathrm{C}(10)-\mathrm{C}(9)-\mathrm{C}(8)$ & $120.1(3)$ \\
\hline $\mathrm{C}(11)-\mathrm{C}(10)-\mathrm{C}(9)$ & $119.4(3)$ \\
\hline$C(10)-C(11)-C(12)$ & $120.2(2)$ \\
\hline$C(7)-C(12)-C(11)$ & $121.1(2)$ \\
\hline $\mathrm{O}(2)-\mathrm{C}(13)-\mathrm{C}(19)$ & $111.22(19)$ \\
\hline $\mathrm{O}(2)-\mathrm{C}(13)-\mathrm{C}(14)$ & $109.77(19)$ \\
\hline$C(19)-C(13)-C(14)$ & $110.88(19)$ \\
\hline $\mathrm{O}(2)-\mathrm{C}(13)-\mathrm{C}(18)$ & $105.77(19)$ \\
\hline $\mathrm{C}(19)-\mathrm{C}(13)-\mathrm{C}(18)$ & $110.51(18)$ \\
\hline$C(14)-C(13)-C(18)$ & $108.53(19)$ \\
\hline $\mathrm{F}(2)-\mathrm{C}(14)-\mathrm{C}(15)$ & $108.0(2)$ \\
\hline $\mathrm{F}(2)-\mathrm{C}(14)-\mathrm{C}(13)$ & $107.46(19)$ \\
\hline$C(15)-C(14)-C(13)$ & $113.4(2)$ \\
\hline $\mathrm{C}(14)-\mathrm{C}(15)-\mathrm{C}(16)$ & $110.4(2)$ \\
\hline$C(15)-C(16)-C(17)$ & $111.2(2)$ \\
\hline $\mathrm{C}(16)-\mathrm{C}(17)-\mathrm{C}(18)$ & $110.7(2)$ \\
\hline$C(17)-C(18)-C(13)$ & $112.2(2)$ \\
\hline$C(20)-C(19)-C(24)$ & $118.9(2)$ \\
\hline$C(20)-C(19)-C(13)$ & $120.4(2)$ \\
\hline $\mathrm{C}(24)-\mathrm{C}(19)-\mathrm{C}(13)$ & $120.7(2)$ \\
\hline $\mathrm{C}(19)-\mathrm{C}(20)-\mathrm{C}(21)$ & $120.4(3)$ \\
\hline$C(22)-C(21)-C(20)$ & $120.2(3)$ \\
\hline
\end{tabular}


$\mathrm{C}(23)-\mathrm{C}(22)-\mathrm{C}(21)$

$120.0(3)$

$\mathrm{C}(22)-\mathrm{C}(23)-\mathrm{C}(24)$

120.1(3)

C(19)-C(24)-C(23)

$120.4(3)$ 
Table 4. Anisotropic displacement parameters $\left(\AA^{2} \times 10^{3}\right)$ for 33. The anisotropic displacement factor exponent takes the form: $-2 \square^{2}\left[h^{2} a^{* 2} U^{11}+\ldots+2 h k a^{*} b^{*} U^{12}\right]$

\begin{tabular}{|c|c|c|c|c|c|c|}
\hline & $\mathrm{U}^{11}$ & $\mathrm{U}^{22}$ & $\mathrm{U}^{33}$ & $\mathrm{U}^{23}$ & $\mathrm{U}^{13}$ & $\mathrm{U}^{12}$ \\
\hline $\mathrm{C}(1)$ & $22(1)$ & $16(1)$ & $22(1)$ & $1(1)$ & $0(1)$ & $1(1)$ \\
\hline$C(2)$ & $21(1)$ & $23(1)$ & $22(1)$ & $-3(1)$ & $-3(1)$ & $0(1)$ \\
\hline$C(3)$ & $24(1)$ & $26(1)$ & $20(1)$ & $-5(1)$ & $4(1)$ & $2(1)$ \\
\hline$C(4)$ & $22(1)$ & $27(1)$ & $35(2)$ & $3(1)$ & $5(1)$ & $4(1)$ \\
\hline$C(5)$ & $20(1)$ & $32(2)$ & $35(2)$ & $2(1)$ & $-3(1)$ & $-5(1)$ \\
\hline$C(6)$ & $26(1)$ & $30(2)$ & $21(1)$ & $-3(1)$ & $-3(1)$ & $-4(1)$ \\
\hline$C(7)$ & $20(1)$ & $26(1)$ & $17(1)$ & $-5(1)$ & $-4(1)$ & $0(1)$ \\
\hline $\mathrm{C}(8)$ & $25(1)$ & $25(1)$ & $22(1)$ & $-1(1)$ & $-4(1)$ & $-1(1)$ \\
\hline $\mathrm{C}(9)$ & $35(1)$ & $28(1)$ & $22(1)$ & $0(1)$ & $-2(1)$ & $-5(1)$ \\
\hline$C(10)$ & $30(1)$ & $38(2)$ & $22(1)$ & $-7(1)$ & $6(1)$ & $-8(1)$ \\
\hline $\mathrm{C}(11)$ & $26(1)$ & $27(1)$ & $34(1)$ & $-8(1)$ & $2(1)$ & $5(1)$ \\
\hline$C(12)$ & $25(1)$ & $22(1)$ & $24(1)$ & $-3(1)$ & $-3(1)$ & $-2(1)$ \\
\hline $\mathrm{O}(1)$ & $36(1)$ & $17(1)$ & $28(1)$ & $3(1)$ & $2(1)$ & $2(1)$ \\
\hline $\mathrm{F}(1)$ & $25(1)$ & $47(1)$ & $30(1)$ & $-12(1)$ & $-6(1)$ & $1(1)$ \\
\hline$C(13)$ & $21(1)$ & $13(1)$ & $16(1)$ & $-1(1)$ & $0(1)$ & $-1(1)$ \\
\hline$C(14)$ & $21(1)$ & $16(1)$ & $23(1)$ & $4(1)$ & $-1(1)$ & $1(1)$ \\
\hline$C(15)$ & $30(1)$ & $19(1)$ & $25(2)$ & $8(1)$ & $3(1)$ & $0(1)$ \\
\hline$C(16)$ & $21(1)$ & $28(1)$ & $34(2)$ & $5(1)$ & $4(1)$ & $-3(1)$ \\
\hline$C(17)$ & $19(1)$ & $31(1)$ & $26(1)$ & $1(1)$ & 1(1) & $2(1)$ \\
\hline$C(18)$ & $19(1)$ & $27(1)$ & $21(1)$ & $-2(1)$ & $-1(1)$ & $4(1)$ \\
\hline$C(19)$ & 19(1) & $19(1)$ & $14(1)$ & $5(1)$ & $-4(1)$ & $0(1)$ \\
\hline$C(20)$ & $25(1)$ & $29(1)$ & $19(1)$ & $-3(1)$ & $-2(1)$ & $0(1)$ \\
\hline$C(21)$ & $28(1)$ & $44(2)$ & $18(1)$ & $-2(1)$ & $2(1)$ & $6(1)$ \\
\hline$C(22)$ & $22(1)$ & $46(2)$ & $21(1)$ & $10(1)$ & $4(1)$ & $7(1)$ \\
\hline$C(23)$ & $24(1)$ & $38(2)$ & $32(2)$ & $12(1)$ & $0(1)$ & $-7(1)$ \\
\hline$C(24)$ & $25(1)$ & $24(1)$ & $24(1)$ & $4(1)$ & $-3(1)$ & $-4(1)$ \\
\hline $\mathrm{O}(2)$ & $34(1)$ & $14(1)$ & 19(1) & $-3(1)$ & $0(1)$ & $-1(1)$ \\
\hline $\mathrm{F}(2)$ & $27(1)$ & $40(1)$ & $29(1)$ & $10(1)$ & $-6(1)$ & $0(1)$ \\
\hline
\end{tabular}


Table 5. Hydrogen coordinates ( $\left.\times 10^{4}\right)$ and isotropic displacement parameters $\left(\AA^{2} \times 10^{3}\right)$ for 33 .

\begin{tabular}{|c|c|c|c|c|}
\hline & $\mathrm{x}$ & $\mathrm{y}$ & $\mathrm{z}$ & $\mathrm{U}(\mathrm{eq})$ \\
\hline $\mathrm{H}(2 \mathrm{~A})$ & 547 & 6739 & 167 & 26 \\
\hline $\mathrm{H}(3 \mathrm{~A})$ & -162 & 6202 & 1015 & 28 \\
\hline $\mathrm{H}(3 \mathrm{~B})$ & -124 & 3391 & 946 & 28 \\
\hline $\mathrm{H}(4 \mathrm{~A})$ & -1634 & 4729 & 711 & 34 \\
\hline $\mathrm{H}(4 \mathrm{~B})$ & -1205 & 6520 & 274 & 34 \\
\hline $\mathrm{H}(5 \mathrm{~A})$ & -1752 & 3143 & -184 & 35 \\
\hline $\mathrm{H}(5 \mathrm{~B})$ & -1131 & 1471 & 195 & 35 \\
\hline $\mathrm{H}(6 \mathrm{~A})$ & -419 & 4746 & -591 & 31 \\
\hline $\mathrm{H}(6 \mathrm{~B})$ & -369 & 1935 & -643 & 31 \\
\hline $\mathrm{H}(8 \mathrm{~A})$ & 944 & 6943 & -750 & 29 \\
\hline $\mathrm{H}(9 \mathrm{~A})$ & 2111 & 7595 & -1389 & 34 \\
\hline $\mathrm{H}(10 \mathrm{~A})$ & 3278 & 4783 & -1519 & 36 \\
\hline $\mathrm{H}(11 \mathrm{~A})$ & 3267 & 1328 & -1005 & 35 \\
\hline $\mathrm{H}(12 \mathrm{~A})$ & 2090 & 671 & -369 & 28 \\
\hline $\mathrm{H}(1)$ & $1020(20)$ & $1130(70)$ & $427(11)$ & $49(11)$ \\
\hline $\mathrm{H}(14 \mathrm{~A})$ & 1783 & 1718 & 1809 & 24 \\
\hline $\mathrm{H}(15 \mathrm{~A})$ & 2546 & 1576 & 969 & 29 \\
\hline $\mathrm{H}(15 \mathrm{~B})$ & 2592 & -1247 & 992 & 29 \\
\hline $\mathrm{H}(16 \mathrm{~A})$ & 3567 & 1875 & 1711 & 33 \\
\hline $\mathrm{H}(16 \mathrm{~B})$ & 4045 & 268 & 1254 & 33 \\
\hline $\mathrm{H}(17 \mathrm{~A})$ & 4206 & -1474 & 2136 & 30 \\
\hline $\mathrm{H}(17 \mathrm{~B})$ & 3652 & -3191 & 1733 & 30 \\
\hline $\mathrm{H}(18 \mathrm{~A})$ & 2807 & -243 & 2546 & 27 \\
\hline $\mathrm{H}(18 \mathrm{~B})$ & 2854 & -3062 & 2570 & 27 \\
\hline $\mathrm{H}(20 \mathrm{~A})$ & 1394 & 1485 & 2749 & 29 \\
\hline $\mathrm{H}(21 \mathrm{~A})$ & 168 & 1671 & 3375 & 36 \\
\hline $\mathrm{H}(22 \mathrm{~A})$ & -907 & -1390 & 3428 & 36 \\
\hline $\mathrm{H}(23 \mathrm{~A})$ & -781 & -4618 & 2849 & 38 \\
\hline $\mathrm{H}(24 \mathrm{~A})$ & 440 & -4826 & 2220 & 29 \\
\hline $\mathrm{H}(2)$ & $1620(30)$ & $-4050(100)$ & $1439(11)$ & $93(17)$ \\
\hline
\end{tabular}


Table 6. Torsion angles $\left[{ }^{\circ}\right]$ for 33 .

\begin{tabular}{|c|c|}
\hline $\mathrm{O}(1)-\mathrm{C}(1)-\mathrm{C}(2)-\mathrm{F}(1)$ & $60.7(3)$ \\
\hline $\mathrm{C}(7)-\mathrm{C}(1)-\mathrm{C}(2)-\mathrm{F}(1)$ & $-62.4(3)$ \\
\hline$C(6)-C(1)-C(2)-F(1)$ & $175.7(2)$ \\
\hline $\mathrm{O}(1)-\mathrm{C}(1)-\mathrm{C}(2)-\mathrm{C}(3)$ & $-59.5(3)$ \\
\hline$C(7)-C(1)-C(2)-C(3)$ & $177.5(2)$ \\
\hline$C(6)-C(1)-C(2)-C(3)$ & $55.6(3)$ \\
\hline $\mathrm{F}(1)-\mathrm{C}(2)-\mathrm{C}(3)-\mathrm{C}(4)$ & $-178.2(2)$ \\
\hline $\mathrm{C}(1)-\mathrm{C}(2)-\mathrm{C}(3)-\mathrm{C}(4)$ & $-58.1(3)$ \\
\hline$C(2)-C(3)-C(4)-C(5)$ & $57.0(3)$ \\
\hline$C(3)-C(4)-C(5)-C(6)$ & $-56.6(3)$ \\
\hline$C(4)-C(5)-C(6)-C(1)$ & $55.4(3)$ \\
\hline $\mathrm{O}(1)-\mathrm{C}(1)-\mathrm{C}(6)-\mathrm{C}(5)$ & $64.6(3)$ \\
\hline$C(2)-C(1)-C(6)-C(5)$ & $-53.2(3)$ \\
\hline$C(7)-C(1)-C(6)-C(5)$ & $-175.1(2)$ \\
\hline $\mathrm{O}(1)-\mathrm{C}(1)-\mathrm{C}(7)-\mathrm{C}(12)$ & $-2.8(3)$ \\
\hline$C(2)-C(1)-C(7)-C(12)$ & $119.5(3)$ \\
\hline$C(6)-C(1)-C(7)-C(12)$ & $-119.7(2)$ \\
\hline $\mathrm{O}(1)-\mathrm{C}(1)-\mathrm{C}(7)-\mathrm{C}(8)$ & $176.8(2)$ \\
\hline$C(2)-C(1)-C(7)-C(8)$ & $-60.9(3)$ \\
\hline$C(6)-C(1)-C(7)-C(8)$ & $59.9(3)$ \\
\hline $\mathrm{C}(12)-\mathrm{C}(7)-\mathrm{C}(8)-\mathrm{C}(9)$ & $1.2(4)$ \\
\hline $\mathrm{C}(1)-\mathrm{C}(7)-\mathrm{C}(8)-\mathrm{C}(9)$ & $-178.4(2)$ \\
\hline$C(7)-C(8)-C(9)-C(10)$ & $-0.6(4)$ \\
\hline $\mathrm{C}(8)-\mathrm{C}(9)-\mathrm{C}(10)-\mathrm{C}(11)$ & $-0.2(4)$ \\
\hline$C(9)-C(10)-C(11)-C(12)$ & $0.4(4)$ \\
\hline $\mathrm{C}(8)-\mathrm{C}(7)-\mathrm{C}(12)-\mathrm{C}(11)$ & $-1.0(4)$ \\
\hline $\mathrm{C}(1)-\mathrm{C}(7)-\mathrm{C}(12)-\mathrm{C}(11)$ & $178.6(2)$ \\
\hline$C(10)-C(11)-C(12)-C(7)$ & $0.2(4)$ \\
\hline $\mathrm{O}(2)-\mathrm{C}(13)-\mathrm{C}(14)-\mathrm{F}(2)$ & $60.0(2)$ \\
\hline $\mathrm{C}(19)-\mathrm{C}(13)-\mathrm{C}(14)-\mathrm{F}(2)$ & $-63.3(2)$ \\
\hline $\mathrm{C}(18)-\mathrm{C}(13)-\mathrm{C}(14)-\mathrm{F}(2)$ & $175.15(19)$ \\
\hline $\mathrm{O}(2)-\mathrm{C}(13)-\mathrm{C}(14)-\mathrm{C}(15)$ & $-59.3(3)$ \\
\hline$C(19)-C(13)-C(14)-C(15)$ & $177.4(2)$ \\
\hline$C(18)-C(13)-C(14)-C(15)$ & $55.9(3)$ \\
\hline
\end{tabular}




\begin{tabular}{|c|c|}
\hline $\mathrm{F}(2)-\mathrm{C}(14)-\mathrm{C}(15)-\mathrm{C}(16)$ & $-176.2(2)$ \\
\hline$C(13)-C(14)-C(15)-C(16)$ & $-57.3(3)$ \\
\hline$C(14)-C(15)-C(16)-C(17)$ & $55.9(3)$ \\
\hline $\mathrm{C}(15)-\mathrm{C}(16)-\mathrm{C}(17)-\mathrm{C}(18)$ & $-55.5(3)$ \\
\hline $\mathrm{C}(16)-\mathrm{C}(17)-\mathrm{C}(18)-\mathrm{C}(13)$ & $55.7(3)$ \\
\hline $\mathrm{O}(2)-\mathrm{C}(13)-\mathrm{C}(18)-\mathrm{C}(17)$ & $63.1(3)$ \\
\hline $\mathrm{C}(19)-\mathrm{C}(13)-\mathrm{C}(18)-\mathrm{C}(17)$ & $-176.4(2)$ \\
\hline $\mathrm{C}(14)-\mathrm{C}(13)-\mathrm{C}(18)-\mathrm{C}(17)$ & $-54.6(3)$ \\
\hline $\mathrm{O}(2)-\mathrm{C}(13)-\mathrm{C}(19)-\mathrm{C}(20)$ & $175.3(2)$ \\
\hline$C(14)-C(13)-C(19)-C(20)$ & $-62.3(3)$ \\
\hline$C(18)-C(13)-C(19)-C(20)$ & $58.1(3)$ \\
\hline $\mathrm{O}(2)-\mathrm{C}(13)-\mathrm{C}(19)-\mathrm{C}(24)$ & $-5.1(3)$ \\
\hline$C(14)-C(13)-C(19)-C(24)$ & $117.3(2)$ \\
\hline $\mathrm{C}(18)-\mathrm{C}(13)-\mathrm{C}(19)-\mathrm{C}(24)$ & $-122.3(2)$ \\
\hline$C(24)-C(19)-C(20)-C(21)$ & $1.0(4)$ \\
\hline$C(13)-C(19)-C(20)-C(21)$ & $-179.4(2)$ \\
\hline$C(19)-C(20)-C(21)-C(22)$ & $-0.2(4)$ \\
\hline$C(20)-C(21)-C(22)-C(23)$ & $-0.6(4)$ \\
\hline$C(21)-C(22)-C(23)-C(24)$ & $0.6(4)$ \\
\hline$C(20)-C(19)-C(24)-C(23)$ & $-1.0(4)$ \\
\hline$C(13)-C(19)-C(24)-C(23)$ & $179.3(2)$ \\
\hline $\mathrm{C}(22)-\mathrm{C}(23)-\mathrm{C}(24)-\mathrm{C}(19)$ & $0.3(4)$ \\
\hline
\end{tabular}


Table 1. Crystal data and structure refinement for Cyclohexanol $\mathbf{3 8 .}$

Identification code

Empirical formula

Formula weight

Temperature

Wavelength

Crystal system

Space group

Unit cell dimensions

Volume

Z

Density (calculated)

Absorption coefficient

$\mathrm{F}(000)$

Crystal color

Crystal size

Theta range for data collection

Index ranges

Reflections collected

Independent reflections

Completeness to theta $=28.29^{\circ}$

Absorption correction

Max. and min. transmission

Refinement method

Data / restraints / parameters

Goodness-of-fit on $\mathrm{F}^{2}$

Final $R$ indices $[I>2 \operatorname{sigma}(I)=3068$ data $]$

$\mathrm{R}$ indices (all data)

Largest diff. peak and hole
Cyclohexanol 38

$\mathrm{C}_{17} \mathrm{H}_{26} \mathrm{O}_{2} \mathrm{~S}$

294.44

163(2) K

$0.71073 \AA$

Triclinic

$P$

$\mathrm{a}=6.1056(7) \AA$

$\square=93.171(2)^{\circ}$.

$\mathrm{b}=11.1995(14) \AA$

$\square=101.992(2)^{\circ}$.

$\mathrm{c}=12.5523(15) \AA$

$\square=105.393(2)^{\circ}$.

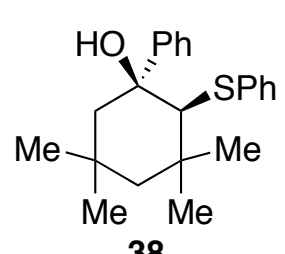

38

803.90(17) $\AA^{3}$

2

$1.216 \mathrm{Mg} / \mathrm{m}^{3}$

$0.201 \mathrm{~mm}^{-1}$

320

colorless

$0.18 \times 0.15 \times 0.14 \mathrm{~mm}^{3}$

1.67 to $28.29^{\circ}$.

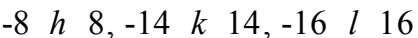

10321

$3906[\mathrm{R}(\mathrm{int})=0.0287]$

$98.2 \%$

Semi-empirical from equivalents

0.9724 and 0.9647

Full-matrix least-squares on $\mathrm{F}^{2}$

3906 / 0 / 285

1.049

$\mathrm{R} 1=0.0386, \mathrm{wR} 2=0.0957$

$\mathrm{R} 1=0.0554, \mathrm{wR} 2=0.1070$

0.470 and -0.248 e. $\AA^{-3}$ 
Table 2. Atomic coordinates $\left(\mathrm{x} 10^{4}\right)$ and equivalent isotropic displacement parameters $\left(\AA^{2} \times 10^{3}\right)$ for kaw78. $U(e q)$ is defined as one third of the trace of the orthogonalized $U^{i j}$ tensor.

\begin{tabular}{|c|c|c|c|c|}
\hline & $\mathrm{x}$ & $\mathrm{y}$ & $\mathrm{z}$ & $\mathrm{U}(\mathrm{eq})$ \\
\hline $\mathrm{S}(1)$ & $3702(1)$ & $3564(1)$ & 1001(1) & $23(1)$ \\
\hline $\mathrm{O}(1)$ & $2447(2)$ & $1646(1)$ & $2721(1)$ & $26(1)$ \\
\hline $\mathrm{O}(2)$ & $1224(2)$ & $3028(1)$ & 1113(1) & $31(1)$ \\
\hline $\mathrm{C}(1)$ & $5665(2)$ & 2902(1) & 1977(1) & $17(1)$ \\
\hline$C(2)$ & $4402(2)$ & $1604(1)$ & $2269(1)$ & $19(1)$ \\
\hline$C(3)$ & 6092(3) & $1184(1)$ & $3142(1)$ & $21(1)$ \\
\hline$C(4)$ & $7408(2)$ & $2106(1)$ & $4170(1)$ & $20(1)$ \\
\hline$C(5)$ & $8536(2)$ & $3359(1)$ & $3796(1)$ & $20(1)$ \\
\hline$C(6)$ & $6998(2)$ & $3930(1)$ & $2957(1)$ & $18(1)$ \\
\hline$C(7)$ & $3577(3)$ & $626(2)$ & $1258(1)$ & $27(1)$ \\
\hline $\mathrm{C}(8)$ & $9367(3)$ & $1609(2)$ & 4792(1) & $29(1)$ \\
\hline $\mathrm{C}(9)$ & $5851(3)$ & $2213(2)$ & $4964(1)$ & $26(1)$ \\
\hline $\mathrm{C}(10)$ & $5367(3)$ & $4502(2)$ & $3468(1)$ & $25(1)$ \\
\hline $\mathrm{C}(11)$ & $8663(3)$ & 4991(2) & $2539(1)$ & $25(1)$ \\
\hline $\mathrm{C}(12)$ & $3832(3)$ & $2874(1)$ & $-299(1)$ & $20(1)$ \\
\hline$C(13)$ & $5908(3)$ & $3185(2)$ & $-652(1)$ & $25(1)$ \\
\hline$C(14)$ & $5881(3)$ & $2755(2)$ & $-1714(1)$ & $28(1)$ \\
\hline$C(15)$ & $3823(3)$ & $2024(2)$ & $-2407(1)$ & $28(1)$ \\
\hline$C(16)$ & $1776(3)$ & $1710(2)$ & $-2044(1)$ & $27(1)$ \\
\hline$C(17)$ & $1764(3)$ & $2147(2)$ & $-988(1)$ & $23(1)$ \\
\hline
\end{tabular}


Table 3. Bond lengths $[\AA]$ and angles $\left[{ }^{\circ}\right]$ for kaw78.

\begin{tabular}{|c|c|}
\hline $\mathrm{S}(1)-\mathrm{O}(2)$ & $1.5116(12)$ \\
\hline$S(1)-C(12)$ & $1.7928(15)$ \\
\hline $\mathrm{S}(1)-\mathrm{C}(1)$ & $1.8570(14)$ \\
\hline $\mathrm{O}(1)-\mathrm{C}(2)$ & $1.4347(17)$ \\
\hline $\mathrm{C}(1)-\mathrm{C}(2)$ & $1.557(2)$ \\
\hline$C(1)-C(6)$ & $1.5590(19)$ \\
\hline$C(2)-C(3)$ & $1.528(2)$ \\
\hline $\mathrm{C}(2)-\mathrm{C}(7)$ & $1.532(2)$ \\
\hline$C(3)-C(4)$ & $1.536(2)$ \\
\hline$C(4)-C(9)$ & $1.534(2)$ \\
\hline$C(4)-C(5)$ & $1.536(2)$ \\
\hline $\mathrm{C}(4)-\mathrm{C}(8)$ & $1.537(2)$ \\
\hline$C(5)-C(6)$ & $1.544(2)$ \\
\hline $\mathrm{C}(6)-\mathrm{C}(11)$ & $1.539(2)$ \\
\hline$C(6)-C(10)$ & $1.540(2)$ \\
\hline$C(12)-C(17)$ & $1.388(2)$ \\
\hline $\mathrm{C}(12)-\mathrm{C}(13)$ & $1.393(2)$ \\
\hline$C(13)-C(14)$ & $1.388(2)$ \\
\hline$C(14)-C(15)$ & $1.388(3)$ \\
\hline$C(15)-C(16)$ & $1.384(2)$ \\
\hline$C(16)-C(17)$ & $1.389(2)$ \\
\hline $\mathrm{O}(2)-\mathrm{S}(1)-\mathrm{C}(12)$ & $106.00(7)$ \\
\hline $\mathrm{O}(2)-\mathrm{S}(1)-\mathrm{C}(1)$ & $108.83(7)$ \\
\hline $\mathrm{C}(12)-\mathrm{S}(1)-\mathrm{C}(1)$ & $102.11(6)$ \\
\hline$C(2)-C(1)-C(6)$ & $116.72(12)$ \\
\hline $\mathrm{C}(2)-\mathrm{C}(1)-\mathrm{S}(1)$ & $113.06(10)$ \\
\hline$C(6)-C(1)-S(1)$ & $107.70(9)$ \\
\hline $\mathrm{O}(1)-\mathrm{C}(2)-\mathrm{C}(3)$ & $106.75(12)$ \\
\hline $\mathrm{O}(1)-\mathrm{C}(2)-\mathrm{C}(7)$ & $109.36(12)$ \\
\hline$C(3)-C(2)-C(7)$ & $108.40(12)$ \\
\hline $\mathrm{O}(1)-\mathrm{C}(2)-\mathrm{C}(1)$ & $111.92(12)$ \\
\hline$C(3)-C(2)-C(1)$ & $109.32(12)$ \\
\hline$C(7)-C(2)-C(1)$ & $110.95(12)$ \\
\hline
\end{tabular}




\begin{tabular}{|c|c|}
\hline$C(2)-C(3)-C(4)$ & $117.62(12)$ \\
\hline $\mathrm{C}(9)-\mathrm{C}(4)-\mathrm{C}(5)$ & $113.33(13)$ \\
\hline$C(9)-C(4)-C(3)$ & $112.57(13)$ \\
\hline$C(5)-C(4)-C(3)$ & $107.98(12)$ \\
\hline $\mathrm{C}(9)-\mathrm{C}(4)-\mathrm{C}(8)$ & $106.58(13)$ \\
\hline$C(5)-C(4)-C(8)$ & $108.19(12)$ \\
\hline $\mathrm{C}(3)-\mathrm{C}(4)-\mathrm{C}(8)$ & $107.98(13)$ \\
\hline$C(4)-C(5)-C(6)$ & $118.63(12)$ \\
\hline $\mathrm{C}(11)-\mathrm{C}(6)-\mathrm{C}(10)$ & $107.55(13)$ \\
\hline$C(11)-C(6)-C(5)$ & $106.84(12)$ \\
\hline$C(10)-C(6)-C(5)$ & $112.75(13)$ \\
\hline$C(11)-C(6)-C(1)$ & $108.60(12)$ \\
\hline$C(10)-C(6)-C(1)$ & $113.16(12)$ \\
\hline$C(5)-C(6)-C(1)$ & $107.70(11)$ \\
\hline$C(17)-C(12)-C(13)$ & $121.21(14)$ \\
\hline $\mathrm{C}(17)-\mathrm{C}(12)-\mathrm{S}(1)$ & $118.16(12)$ \\
\hline$C(13)-C(12)-S(1)$ & $120.21(12)$ \\
\hline$C(14)-C(13)-C(12)$ & $118.70(15)$ \\
\hline $\mathrm{C}(13)-\mathrm{C}(14)-\mathrm{C}(15)$ & $120.46(16)$ \\
\hline$C(16)-C(15)-C(14)$ & $120.32(15)$ \\
\hline$C(15)-C(16)-C(17)$ & $119.98(16)$ \\
\hline $\mathrm{C}(12)-\mathrm{C}(17)-\mathrm{C}(16)$ & $119.31(15)$ \\
\hline
\end{tabular}


Table 4. Anisotropic displacement parameters $\left(\AA^{2} \times 10^{3}\right)$ for kaw78. The anisotropic displacement factor exponent takes the form: $-2 \square^{2}\left[\mathrm{~h}^{2} \mathrm{a}^{* 2} \mathrm{U}^{11}+\ldots+2 \mathrm{~h} \mathrm{k} \mathrm{a}^{*} \mathrm{~b}^{*} \mathrm{U}^{12}\right]$

\begin{tabular}{|c|c|c|c|c|c|c|}
\hline & $\mathrm{U}^{11}$ & $\mathrm{U}^{22}$ & $\mathrm{U}^{33}$ & $\mathrm{U}^{23}$ & $\mathrm{U}^{13}$ & $\mathrm{U}^{12}$ \\
\hline$S(1)$ & $22(1)$ & $25(1)$ & $22(1)$ & $-1(1)$ & $1(1)$ & $12(1)$ \\
\hline $\mathrm{O}(1)$ & $14(1)$ & $37(1)$ & $26(1)$ & $2(1)$ & $6(1)$ & $3(1)$ \\
\hline $\mathrm{O}(2)$ & $19(1)$ & $52(1)$ & $27(1)$ & $0(1)$ & $4(1)$ & $18(1)$ \\
\hline$C(1)$ & $14(1)$ & $19(1)$ & 18(1) & $0(1)$ & $2(1)$ & $6(1)$ \\
\hline$C(2)$ & $16(1)$ & $21(1)$ & $20(1)$ & $0(1)$ & $6(1)$ & $3(1)$ \\
\hline$C(3)$ & $22(1)$ & $19(1)$ & 23(1) & $3(1)$ & $7(1)$ & $5(1)$ \\
\hline$C(4)$ & $15(1)$ & $27(1)$ & 19(1) & $2(1)$ & $4(1)$ & $6(1)$ \\
\hline$C(5)$ & $13(1)$ & $25(1)$ & $20(1)$ & $0(1)$ & $3(1)$ & $4(1)$ \\
\hline$C(6)$ & $15(1)$ & $18(1)$ & 21(1) & $-2(1)$ & $3(1)$ & $4(1)$ \\
\hline$C(7)$ & $33(1)$ & $19(1)$ & $24(1)$ & $-1(1)$ & $3(1)$ & $2(1)$ \\
\hline$C(8)$ & $23(1)$ & $38(1)$ & $27(1)$ & $10(1)$ & $6(1)$ & $13(1)$ \\
\hline$C(9)$ & $20(1)$ & $38(1)$ & 21(1) & $1(1)$ & $8(1)$ & $6(1)$ \\
\hline$C(10)$ & $21(1)$ & $27(1)$ & $27(1)$ & $-6(1)$ & $4(1)$ & $10(1)$ \\
\hline $\mathrm{C}(11)$ & $23(1)$ & $20(1)$ & $29(1)$ & $1(1)$ & $4(1)$ & $2(1)$ \\
\hline$C(12)$ & $20(1)$ & $22(1)$ & 20(1) & $3(1)$ & $3(1)$ & $9(1)$ \\
\hline$C(13)$ & $21(1)$ & $26(1)$ & $29(1)$ & $8(1)$ & $5(1)$ & $8(1)$ \\
\hline$C(14)$ & $27(1)$ & $36(1)$ & $31(1)$ & $14(1)$ & 14(1) & $16(1)$ \\
\hline$C(15)$ & $39(1)$ & $31(1)$ & 21(1) & $7(1)$ & 10(1) & $18(1)$ \\
\hline$C(16)$ & $28(1)$ & $29(1)$ & 23(1) & $2(1)$ & $2(1)$ & $8(1)$ \\
\hline$C(17)$ & $20(1)$ & $28(1)$ & 21(1) & $3(1)$ & $3(1)$ & $7(1)$ \\
\hline
\end{tabular}


Table 5. Hydrogen coordinates $\left(\mathrm{x} 10^{4}\right)$ and isotropic displacement parameters $\left(\AA^{2} \mathrm{x} 10^{3}\right)$ for kaw78.

\begin{tabular}{|c|c|c|c|c|}
\hline & $\mathrm{x}$ & $\mathrm{y}$ & Z & $\mathrm{U}(\mathrm{eq})$ \\
\hline $\mathrm{H}(1)$ & $1610(40)$ & $1940(20)$ & $2270(20)$ & $49(7)$ \\
\hline $\mathrm{H}(1 \mathrm{~A})$ & $6790(30)$ & $2760(15)$ & $1541(14)$ & $18(4)$ \\
\hline $\mathrm{H}(3 \mathrm{~A})$ & $5170(30)$ & $378(18)$ & $3345(15)$ & $28(5)$ \\
\hline $\mathrm{H}(3 \mathrm{~B})$ & $7210(30)$ & $975(16)$ & $2782(15)$ & $26(5)$ \\
\hline $\mathrm{H}(5 \mathrm{~A})$ & $9260(30)$ & $3987(17)$ & $4446(15)$ & $23(4)$ \\
\hline $\mathrm{H}(5 \mathrm{~B})$ & $9750(30)$ & $3237(15)$ & $3476(14)$ & $19(4)$ \\
\hline $\mathrm{H}(7 \mathrm{~A})$ & $2260(30)$ & $739(18)$ & $730(16)$ & $33(5)$ \\
\hline $\mathrm{H}(7 \mathrm{~B})$ & $3120(30)$ & $-183(19)$ & 1494(16) & $35(5)$ \\
\hline $\mathrm{H}(7 \mathrm{C})$ & $4890(40)$ & $662(18)$ & $886(17)$ & $38(5)$ \\
\hline $\mathrm{H}(8 \mathrm{~A})$ & $10310(40)$ & 2207(19) & $5449(17)$ & $36(5)$ \\
\hline $\mathrm{H}(8 \mathrm{~B})$ & $8730(30)$ & $830(18)$ & $5078(16)$ & $30(5)$ \\
\hline $\mathrm{H}(8 \mathrm{C})$ & $10410(40)$ & 1471(19) & $4323(16)$ & $36(5)$ \\
\hline $\mathrm{H}(9 \mathrm{~A})$ & $6780(30)$ & $2828(19)$ & $5602(17)$ & $35(5)$ \\
\hline $\mathrm{H}(9 \mathrm{~B})$ & $5360(40)$ & $1380(20)$ & $5227(18)$ & $44(6)$ \\
\hline $\mathrm{H}(9 \mathrm{C})$ & $4480(40)$ & $2478(18)$ & $4629(16)$ & $36(5)$ \\
\hline $\mathrm{H}(10 \mathrm{~A})$ & $6260(40)$ & $5070(19)$ & $4109(17)$ & $38(5)$ \\
\hline $\mathrm{H}(10 \mathrm{~B})$ & $4610(30)$ & 4991(17) & $2965(15)$ & $27(5)$ \\
\hline $\mathrm{H}(10 \mathrm{C})$ & $4050(40)$ & $3880(20)$ & $3677(17)$ & $43(6)$ \\
\hline $\mathrm{H}(11 \mathrm{~A})$ & $7890(30)$ & $5420(18)$ & $2023(16)$ & $31(5)$ \\
\hline $\mathrm{H}(11 \mathrm{~B})$ & $9630(30)$ & $5628(18)$ & $3155(15)$ & $30(5)$ \\
\hline $\mathrm{H}(11 \mathrm{C})$ & $9730(30)$ & $4694(17)$ & $2175(15)$ & $29(5)$ \\
\hline $\mathrm{H}(13)$ & $7340(30)$ & $3692(18)$ & $-169(16)$ & $31(5)$ \\
\hline $\mathrm{H}(14)$ & $7180(40)$ & 2976(19) & $-1966(16)$ & $37(5)$ \\
\hline $\mathrm{H}(15)$ & $3860(30)$ & $1772(18)$ & $-3128(17)$ & $34(5)$ \\
\hline $\mathrm{H}(16)$ & $380(40)$ & 1203(19) & $-2527(17)$ & $39(5)$ \\
\hline $\mathrm{H}(17)$ & $380(30)$ & $1978(17)$ & $-713(15)$ & $29(5)$ \\
\hline
\end{tabular}


Table 6. Torsion angles $\left[{ }^{\circ}\right]$ for kaw78.

\begin{tabular}{|c|c|}
\hline $\mathrm{O}(2)-\mathrm{S}(1)-\mathrm{C}(1)-\mathrm{C}(2)$ & $25.97(12)$ \\
\hline$C(12)-S(1)-C(1)-C(2)$ & $-85.81(11)$ \\
\hline $\mathrm{O}(2)-\mathrm{S}(1)-\mathrm{C}(1)-\mathrm{C}(6)$ & $-104.49(10)$ \\
\hline $\mathrm{C}(12)-\mathrm{S}(1)-\mathrm{C}(1)-\mathrm{C}(6)$ & $143.73(10)$ \\
\hline $\mathrm{C}(6)-\mathrm{C}(1)-\mathrm{C}(2)-\mathrm{O}(1)$ & $68.43(15)$ \\
\hline $\mathrm{S}(1)-\mathrm{C}(1)-\mathrm{C}(2)-\mathrm{O}(1)$ & $-57.31(14)$ \\
\hline $\mathrm{C}(6)-\mathrm{C}(1)-\mathrm{C}(2)-\mathrm{C}(3)$ & $-49.62(16)$ \\
\hline $\mathrm{S}(1)-\mathrm{C}(1)-\mathrm{C}(2)-\mathrm{C}(3)$ & $-175.37(10)$ \\
\hline $\mathrm{C}(6)-\mathrm{C}(1)-\mathrm{C}(2)-\mathrm{C}(7)$ & $-169.11(12)$ \\
\hline $\mathrm{S}(1)-\mathrm{C}(1)-\mathrm{C}(2)-\mathrm{C}(7)$ & $65.14(14)$ \\
\hline $\mathrm{O}(1)-\mathrm{C}(2)-\mathrm{C}(3)-\mathrm{C}(4)$ & $-70.52(16)$ \\
\hline $\mathrm{C}(7)-\mathrm{C}(2)-\mathrm{C}(3)-\mathrm{C}(4)$ & $171.77(13)$ \\
\hline $\mathrm{C}(1)-\mathrm{C}(2)-\mathrm{C}(3)-\mathrm{C}(4)$ & $50.72(16)$ \\
\hline $\mathrm{C}(2)-\mathrm{C}(3)-\mathrm{C}(4)-\mathrm{C}(9)$ & $75.17(17)$ \\
\hline $\mathrm{C}(2)-\mathrm{C}(3)-\mathrm{C}(4)-\mathrm{C}(5)$ & $-50.70(17)$ \\
\hline $\mathrm{C}(2)-\mathrm{C}(3)-\mathrm{C}(4)-\mathrm{C}(8)$ & $-167.47(13)$ \\
\hline $\mathrm{C}(9)-\mathrm{C}(4)-\mathrm{C}(5)-\mathrm{C}(6)$ & $-74.21(17)$ \\
\hline $\mathrm{C}(3)-\mathrm{C}(4)-\mathrm{C}(5)-\mathrm{C}(6)$ & $51.20(17)$ \\
\hline $\mathrm{C}(8)-\mathrm{C}(4)-\mathrm{C}(5)-\mathrm{C}(6)$ & $167.83(13)$ \\
\hline $\mathrm{C}(4)-\mathrm{C}(5)-\mathrm{C}(6)-\mathrm{C}(11)$ & $-167.09(13)$ \\
\hline$C(4)-C(5)-C(6)-C(10)$ & $74.97(17)$ \\
\hline $\mathrm{C}(4)-\mathrm{C}(5)-\mathrm{C}(6)-\mathrm{C}(1)$ & $-50.58(17)$ \\
\hline$C(2)-C(1)-C(6)-C(11)$ & $164.15(12)$ \\
\hline$S(1)-C(1)-C(6)-C(11)$ & $-67.46(13)$ \\
\hline $\mathrm{C}(2)-\mathrm{C}(1)-\mathrm{C}(6)-\mathrm{C}(10)$ & $-76.51(16)$ \\
\hline$S(1)-C(1)-C(6)-C(10)$ & $51.87(14)$ \\
\hline $\mathrm{C}(2)-\mathrm{C}(1)-\mathrm{C}(6)-\mathrm{C}(5)$ & $48.80(16)$ \\
\hline $\mathrm{S}(1)-\mathrm{C}(1)-\mathrm{C}(6)-\mathrm{C}(5)$ & $177.19(9)$ \\
\hline $\mathrm{O}(2)-\mathrm{S}(1)-\mathrm{C}(12)-\mathrm{C}(17)$ & $7.15(14)$ \\
\hline $\mathrm{C}(1)-\mathrm{S}(1)-\mathrm{C}(12)-\mathrm{C}(17)$ & $121.04(12)$ \\
\hline $\mathrm{O}(2)-\mathrm{S}(1)-\mathrm{C}(12)-\mathrm{C}(13)$ & $179.76(12)$ \\
\hline$C(1)-S(1)-C(12)-C(13)$ & $-66.36(13)$ \\
\hline$C(17)-C(12)-C(13)-C(14)$ & $0.1(2)$ \\
\hline$S(1)-C(12)-C(13)-C(14)$ & $-172.27(12)$ \\
\hline
\end{tabular}


$\mathrm{C}(12)-\mathrm{C}(13)-\mathrm{C}(14)-\mathrm{C}(15)$

$-0.3(2)$

$\mathrm{C}(13)-\mathrm{C}(14)-\mathrm{C}(15)-\mathrm{C}(16)$

$-0.4(2)$

$\mathrm{C}(14)-\mathrm{C}(15)-\mathrm{C}(16)-\mathrm{C}(17)$

1.4(2)

$\mathrm{C}(13)-\mathrm{C}(12)-\mathrm{C}(17)-\mathrm{C}(16)$

$0.8(2)$

$\mathrm{S}(1)-\mathrm{C}(12)-\mathrm{C}(17)-\mathrm{C}(16)$

$173.35(12)$

$\mathrm{C}(15)-\mathrm{C}(16)-\mathrm{C}(17)-\mathrm{C}(12)$

$-1.5(2)$ 
Table 1. Crystal data and structure refinement for Sulfoxide S-12.

Identification code

Empirical formula

Formula weight

Temperature

Wavelength

Crystal system

Space group

Unit cell dimensions

Volume

Z

Density (calculated)

Absorption coefficient

$\mathrm{F}(000)$

Crystal color

Crystal size

Theta range for data collection

Index ranges

Reflections collected

Independent reflections

Completeness to theta $=28.32^{\circ}$

Absorption correction

Max. and min. transmission

Refinement method

Data / restraints / parameters

Goodness-of-fit on $\mathrm{F}^{2}$

Final $R$ indices $[I>2 \operatorname{sigma}(I)=8208$ data $]$

$\mathrm{R}$ indices (all data)

Absolute structure parameter

Largest diff. peak and hole
Sulfoxide S-12

$\mathrm{C}_{22} \mathrm{H}_{28} \mathrm{O} \mathrm{S}$

340.50

163(2) K

$0.71073 \AA$

Orthorhombic

$P_{c a 2}$

$\mathrm{a}=16.8009(18) \AA$

$\square=90^{\circ}$

$\mathrm{b}=11.0584(12) \AA$

$\square=90^{\circ}$.

$\mathrm{c}=20.623(2) \AA$

$\square=90^{\circ}$.

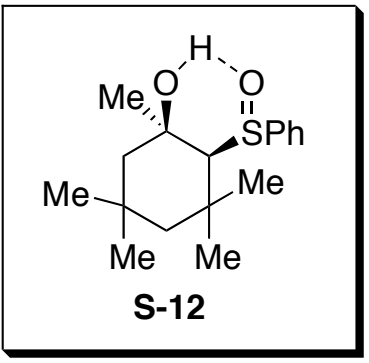

$3831.6(7) \AA^{3}$

8

$1.181 \mathrm{Mg} / \mathrm{m}^{3}$

$0.174 \mathrm{~mm}^{-1}$

1472

pale yellow

$0.39 \times 0.26 \times 0.21 \mathrm{~mm}^{3}$

1.84 to $28.32^{\circ}$.

$\begin{array}{llllllll}-22 & h & 22,-14 & k & 14,-27 & l & 27\end{array}$

47135

$9472[\mathrm{R}($ int $)=0.0440]$

$99.8 \%$

Semi-empirical from equivalents

0.9643 and 0.9351

Full-matrix least-squares on $\mathrm{F}^{2}$

$9472 / 1 / 658$

1.048

$\mathrm{R} 1=0.0335, \mathrm{wR} 2=0.0729$

$\mathrm{R} 1=0.0467, \mathrm{wR} 2=0.0808$

$0.20(4)$

0.290 and -0.163 e. $\AA^{-3}$ 
Table 2. Atomic coordinates $\left(\mathrm{x} 10^{4}\right)$ and equivalent isotropic displacement parameters $\left(\AA^{2} \times 10^{3}\right)$ for kaw82. $U(e q)$ is defined as one third of the trace of the orthogonalized $U^{i j}$ tensor.

\begin{tabular}{|c|c|c|c|c|}
\hline & $\mathrm{x}$ & $\mathrm{y}$ & z & $\mathrm{U}(\mathrm{eq})$ \\
\hline $\mathrm{S}(1)$ & $9062(1)$ & 1918(1) & 5994(1) & $24(1)$ \\
\hline $\mathrm{O}(1)$ & $8164(1)$ & $642(1)$ & $4905(1)$ & $24(1)$ \\
\hline$C(1)$ & $8119(1)$ & 1079(2) & $6075(1)$ & $19(1)$ \\
\hline$C(2)$ & $7669(1)$ & 1089(2) & $5416(1)$ & $19(1)$ \\
\hline$C(3)$ & 6944(1) & $244(2)$ & $5434(1)$ & $23(1)$ \\
\hline $\mathrm{C}(4)$ & $7075(1)$ & $-1039(2)$ & $5699(1)$ & $25(1)$ \\
\hline$C(5)$ & $7507(1)$ & $-924(2)$ & $6354(1)$ & $24(1)$ \\
\hline$C(6)$ & $8285(1)$ & $-181(2)$ & $6379(1)$ & $21(1)$ \\
\hline$C(7)$ & $7500(1)$ & $-1874(2)$ & $5224(1)$ & $32(1)$ \\
\hline $\mathrm{C}(8)$ & $6247(1)$ & $-1594(2)$ & $5825(1)$ & $38(1)$ \\
\hline $\mathrm{C}(9)$ & 8999(1) & $-834(2)$ & $6068(1)$ & $27(1)$ \\
\hline$C(10)$ & $8483(1)$ & $1(2)$ & $7102(1)$ & $30(1)$ \\
\hline $\mathrm{C}(11)$ & $8831(1)$ & $3407(2)$ & $6259(1)$ & $26(1)$ \\
\hline$C(12)$ & $9132(2)$ & 4368(2) & $5908(1)$ & $41(1)$ \\
\hline$C(13)$ & $9038(2)$ & $5537(2)$ & $6136(1)$ & $58(1)$ \\
\hline$C(14)$ & $8636(2)$ & $5747(2)$ & $6705(1)$ & $53(1)$ \\
\hline$C(15)$ & $8320(2)$ & 4795(2) & 7051(1) & $42(1)$ \\
\hline$C(16)$ & $8431(1)$ & $3615(2)$ & 6833(1) & $31(1)$ \\
\hline$C(17)$ & $7400(1)$ & 2389(2) & $5275(1)$ & $23(1)$ \\
\hline$C(18)$ & $7706(1)$ & 3044(2) & $4755(1)$ & $27(1)$ \\
\hline $\mathrm{C}(19)$ & $7475(1)$ & $4235(2)$ & $4644(1)$ & $37(1)$ \\
\hline$C(20)$ & $6925(2)$ & 4781(2) & $5044(1)$ & $42(1)$ \\
\hline$C(21)$ & $6608(1)$ & $4150(2)$ & $5559(1)$ & $40(1)$ \\
\hline$C(22)$ & $6850(1)$ & 2966(2) & $5676(1)$ & $31(1)$ \\
\hline $\mathrm{S}(2)$ & $5070(1)$ & $94(1)$ & $9318(1)$ & $21(1)$ \\
\hline $\mathrm{O}(2)$ & $6605(1)$ & $-1124(1)$ & 8791(1) & $21(1)$ \\
\hline$C(31)$ & $5162(1)$ & $-904(2)$ & $8605(1)$ & $18(1)$ \\
\hline$C(32)$ & $6019(1)$ & $-827(2)$ & $8317(1)$ & $19(1)$ \\
\hline$C(33)$ & $6120(1)$ & $-1755(2)$ & 7763(1) & $21(1)$ \\
\hline$C(34)$ & $5857(1)$ & $-3067(2)$ & 7899(1) & $21(1)$ \\
\hline$C(35)$ & $5016(1)$ & $-3022(2)$ & $8187(1)$ & $22(1)$ \\
\hline
\end{tabular}




\begin{tabular}{lrrrl}
$\mathrm{C}(36)$ & $4860(1)$ & $-2199(2)$ & $8777(1)$ & $22(1)$ \\
$\mathrm{C}(37)$ & $5798(1)$ & $-3733(2)$ & $7247(1)$ & $28(1)$ \\
$\mathrm{C}(38)$ & $6452(1)$ & $-3765(2)$ & $8321(1)$ & $27(1)$ \\
$\mathrm{C}(39)$ & $3955(1)$ & $-2143(2)$ & $8859(1)$ & $32(1)$ \\
$\mathrm{C}(40)$ & $5215(1)$ & $-2688(2)$ & $9411(1)$ & $29(1)$ \\
$\mathrm{C}(41)$ & $4620(1)$ & $1423(2)$ & $9002(1)$ & $21(1)$ \\
$\mathrm{C}(42)$ & $3883(1)$ & $1389(2)$ & $8701(1)$ & $28(1)$ \\
$\mathrm{C}(43)$ & $3522(1)$ & $2458(2)$ & $8500(1)$ & $37(1)$ \\
$\mathrm{C}(44)$ & $3887(2)$ & $3548(2)$ & $8607(1)$ & $41(1)$ \\
$\mathrm{C}(45)$ & $4621(2)$ & $3588(2)$ & $8920(1)$ & $42(1)$ \\
$\mathrm{C}(46)$ & $4985(1)$ & $2530(2)$ & $9110(1)$ & $31(1)$ \\
$\mathrm{C}(47)$ & $6161(1)$ & $445(2)$ & $8041(1)$ & $19(1)$ \\
$\mathrm{C}(48)$ & $6784(1)$ & $1169(2)$ & $8258(1)$ & $22(1)$ \\
$\mathrm{C}(49)$ & $6900(1)$ & $2330(2)$ & $8005(1)$ & $27(1)$ \\
$\mathrm{C}(50)$ & $6405(1)$ & $2776(2)$ & $7532(1)$ & $29(1)$ \\
$\mathrm{C}(51)$ & $5781(1)$ & $2066(2)$ & $7306(1)$ & $29(1)$ \\
$\mathrm{C}(52)$ & $5665(1)$ & $913(2)$ & $7557(1)$ & $25(1)$ \\
& & & & \\
\hline
\end{tabular}


Table 3. Bond lengths $[\AA]$ and angles $\left[{ }^{\circ}\right]$ for kaw 82 .

\begin{tabular}{|c|c|}
\hline$S(1)-C(11)$ & $1.7777(19)$ \\
\hline$S(1)-C(1)$ & $1.8438(17)$ \\
\hline $\mathrm{O}(1)-\mathrm{C}(2)$ & $1.431(2)$ \\
\hline$C(1)-C(6)$ & $1.553(2)$ \\
\hline$C(1)-C(2)$ & $1.555(2)$ \\
\hline$C(2)-C(17)$ & $1.534(2)$ \\
\hline$C(2)-C(3)$ & $1.536(2)$ \\
\hline$C(3)-C(4)$ & $1.536(3)$ \\
\hline$C(4)-C(7)$ & $1.525(3)$ \\
\hline$C(4)-C(5)$ & $1.539(2)$ \\
\hline$C(4)-C(8)$ & $1.542(3)$ \\
\hline$C(5)-C(6)$ & $1.546(2)$ \\
\hline$C(6)-C(10)$ & $1.540(2)$ \\
\hline$C(6)-C(9)$ & $1.541(2)$ \\
\hline $\mathrm{C}(11)-\mathrm{C}(16)$ & $1.381(3)$ \\
\hline $\mathrm{C}(11)-\mathrm{C}(12)$ & $1.382(3)$ \\
\hline $\mathrm{C}(12)-\mathrm{C}(13)$ & $1.384(3)$ \\
\hline$C(13)-C(14)$ & $1.374(4)$ \\
\hline$C(14)-C(15)$ & $1.378(4)$ \\
\hline$C(15)-C(16)$ & $1.393(3)$ \\
\hline C(17)-C(18) & $1.393(3)$ \\
\hline $\mathrm{C}(17)-\mathrm{C}(22)$ & $1.395(3)$ \\
\hline$C(18)-C(19)$ & $1.392(3)$ \\
\hline$C(19)-C(20)$ & $1.377(4)$ \\
\hline$C(20)-C(21)$ & $1.378(3)$ \\
\hline$C(21)-C(22)$ & $1.391(3)$ \\
\hline$S(2)-C(41)$ & $1.7767(17)$ \\
\hline $\mathrm{S}(2)-\mathrm{C}(31)$ & $1.8441(17)$ \\
\hline $\mathrm{O}(2)-\mathrm{C}(32)$ & $1.425(2)$ \\
\hline$C(31)-C(36)$ & $1.560(2)$ \\
\hline$C(31)-C(32)$ & $1.560(2)$ \\
\hline$C(32)-C(47)$ & $1.536(2)$ \\
\hline$C(32)-C(33)$ & $1.546(2)$ \\
\hline$C(33)-C(34)$ & $1.542(2)$ \\
\hline
\end{tabular}




\begin{tabular}{|c|c|}
\hline$C(34)-C(35)$ & $1.532(2)$ \\
\hline$C(34)-C(38)$ & $1.535(3)$ \\
\hline$C(34)-C(37)$ & $1.537(2)$ \\
\hline$C(35)-C(36)$ & $1.542(2)$ \\
\hline$C(36)-C(39)$ & $1.530(3)$ \\
\hline$C(36)-C(40)$ & $1.536(3)$ \\
\hline$C(41)-C(42)$ & $1.385(3)$ \\
\hline$C(41)-C(46)$ & $1.387(3)$ \\
\hline$C(42)-C(43)$ & $1.392(3)$ \\
\hline$C(43)-C(44)$ & $1.370(3)$ \\
\hline$C(44)-C(45)$ & $1.393(3)$ \\
\hline$C(45)-C(46)$ & $1.376(3)$ \\
\hline $\mathrm{C}(47)-\mathrm{C}(48)$ & $1.393(2)$ \\
\hline$C(47)-C(52)$ & $1.400(2)$ \\
\hline $\mathrm{C}(48)-\mathrm{C}(49)$ & $1.399(3)$ \\
\hline$C(49)-C(50)$ & $1.373(3)$ \\
\hline$C(50)-C(51)$ & $1.389(3)$ \\
\hline$C(51)-C(52)$ & $1.390(3)$ \\
\hline $\mathrm{C}(11)-\mathrm{S}(1)-\mathrm{C}(1)$ & $104.50(8)$ \\
\hline $\mathrm{C}(6)-\mathrm{C}(1)-\mathrm{C}(2)$ & $116.60(14)$ \\
\hline $\mathrm{C}(6)-\mathrm{C}(1)-\mathrm{S}(1)$ & $109.48(11)$ \\
\hline $\mathrm{C}(2)-\mathrm{C}(1)-\mathrm{S}(1)$ & $109.55(11)$ \\
\hline $\mathrm{O}(1)-\mathrm{C}(2)-\mathrm{C}(17)$ & $110.77(13)$ \\
\hline $\mathrm{O}(1)-\mathrm{C}(2)-\mathrm{C}(3)$ & $105.54(14)$ \\
\hline $\mathrm{C}(17)-\mathrm{C}(2)-\mathrm{C}(3)$ & $109.98(14)$ \\
\hline $\mathrm{O}(1)-\mathrm{C}(2)-\mathrm{C}(1)$ & $110.97(13)$ \\
\hline $\mathrm{C}(17)-\mathrm{C}(2)-\mathrm{C}(1)$ & $108.46(13)$ \\
\hline $\mathrm{C}(3)-\mathrm{C}(2)-\mathrm{C}(1)$ & $111.12(13)$ \\
\hline $\mathrm{C}(2)-\mathrm{C}(3)-\mathrm{C}(4)$ & $117.19(15)$ \\
\hline$C(7)-C(4)-C(3)$ & $113.40(15)$ \\
\hline$C(7)-C(4)-C(5)$ & $113.18(16)$ \\
\hline$C(3)-C(4)-C(5)$ & $107.68(15)$ \\
\hline $\mathrm{C}(7)-\mathrm{C}(4)-\mathrm{C}(8)$ & $106.89(17)$ \\
\hline$C(3)-C(4)-C(8)$ & $107.35(16)$ \\
\hline$C(5)-C(4)-C(8)$ & $108.07(15)$ \\
\hline
\end{tabular}




\begin{tabular}{|c|c|}
\hline$C(4)-C(5)-C(6)$ & $118.21(14)$ \\
\hline$C(10)-C(6)-C(9)$ & $107.28(15)$ \\
\hline$C(10)-C(6)-C(5)$ & $106.50(15)$ \\
\hline$C(9)-C(6)-C(5)$ & $113.29(15)$ \\
\hline$C(10)-C(6)-C(1)$ & $108.24(15)$ \\
\hline$C(9)-C(6)-C(1)$ & $113.12(14)$ \\
\hline$C(5)-C(6)-C(1)$ & $108.10(13)$ \\
\hline$C(16)-C(11)-C(12)$ & $119.92(19)$ \\
\hline$C(16)-C(11)-S(1)$ & $121.61(15)$ \\
\hline$C(12)-C(11)-S(1)$ & $118.15(16)$ \\
\hline$C(11)-C(12)-C(13)$ & $119.9(2)$ \\
\hline $\mathrm{C}(14)-\mathrm{C}(13)-\mathrm{C}(12)$ & $120.3(2)$ \\
\hline$C(13)-C(14)-C(15)$ & $120.2(2)$ \\
\hline$C(14)-C(15)-C(16)$ & $119.8(2)$ \\
\hline$C(11)-C(16)-C(15)$ & $119.9(2)$ \\
\hline $\mathrm{C}(18)-\mathrm{C}(17)-\mathrm{C}(22)$ & $117.57(17)$ \\
\hline $\mathrm{C}(18)-\mathrm{C}(17)-\mathrm{C}(2)$ & $121.71(16)$ \\
\hline$C(22)-C(17)-C(2)$ & $120.71(16)$ \\
\hline $\mathrm{C}(19)-\mathrm{C}(18)-\mathrm{C}(17)$ & $121.1(2)$ \\
\hline $\mathrm{C}(20)-\mathrm{C}(19)-\mathrm{C}(18)$ & $120.2(2)$ \\
\hline$C(19)-C(20)-C(21)$ & $119.9(2)$ \\
\hline $\mathrm{C}(20)-\mathrm{C}(21)-\mathrm{C}(22)$ & $119.8(2)$ \\
\hline$C(21)-C(22)-C(17)$ & $121.4(2)$ \\
\hline $\mathrm{C}(41)-\mathrm{S}(2)-\mathrm{C}(31)$ & $103.82(8)$ \\
\hline$C(36)-C(31)-C(32)$ & $115.93(14)$ \\
\hline $\mathrm{C}(36)-\mathrm{C}(31)-\mathrm{S}(2)$ & 109.94(11) \\
\hline $\mathrm{C}(32)-\mathrm{C}(31)-\mathrm{S}(2)$ & $110.41(11)$ \\
\hline $\mathrm{O}(2)-\mathrm{C}(32)-\mathrm{C}(47)$ & $111.00(13)$ \\
\hline $\mathrm{O}(2)-\mathrm{C}(32)-\mathrm{C}(33)$ & $106.20(14)$ \\
\hline $\mathrm{C}(47)-\mathrm{C}(32)-\mathrm{C}(33)$ & $108.47(13)$ \\
\hline $\mathrm{O}(2)-\mathrm{C}(32)-\mathrm{C}(31)$ & $111.34(13)$ \\
\hline $\mathrm{C}(47)-\mathrm{C}(32)-\mathrm{C}(31)$ & $109.52(13)$ \\
\hline $\mathrm{C}(33)-\mathrm{C}(32)-\mathrm{C}(31)$ & $110.23(13)$ \\
\hline $\mathrm{C}(34)-\mathrm{C}(33)-\mathrm{C}(32)$ & $117.32(14)$ \\
\hline $\mathrm{C}(35)-\mathrm{C}(34)-\mathrm{C}(38)$ & $113.37(15)$ \\
\hline $\mathrm{C}(35)-\mathrm{C}(34)-\mathrm{C}(37)$ & $107.21(15)$ \\
\hline
\end{tabular}




\begin{tabular}{|c|c|}
\hline $\mathrm{C}(38)-\mathrm{C}(34)-\mathrm{C}(37)$ & $107.31(15)$ \\
\hline $\mathrm{C}(35)-\mathrm{C}(34)-\mathrm{C}(33)$ & $107.69(14)$ \\
\hline $\mathrm{C}(38)-\mathrm{C}(34)-\mathrm{C}(33)$ & $112.94(15)$ \\
\hline $\mathrm{C}(37)-\mathrm{C}(34)-\mathrm{C}(33)$ & $108.05(15)$ \\
\hline $\mathrm{C}(34)-\mathrm{C}(35)-\mathrm{C}(36)$ & $118.84(14)$ \\
\hline $\mathrm{C}(39)-\mathrm{C}(36)-\mathrm{C}(40)$ & $107.76(16)$ \\
\hline $\mathrm{C}(39)-\mathrm{C}(36)-\mathrm{C}(35)$ & $106.31(15)$ \\
\hline $\mathrm{C}(40)-\mathrm{C}(36)-\mathrm{C}(35)$ & $113.40(15)$ \\
\hline $\mathrm{C}(39)-\mathrm{C}(36)-\mathrm{C}(31)$ & $108.19(15)$ \\
\hline $\mathrm{C}(40)-\mathrm{C}(36)-\mathrm{C}(31)$ & $112.93(14)$ \\
\hline $\mathrm{C}(35)-\mathrm{C}(36)-\mathrm{C}(31)$ & $107.92(14)$ \\
\hline$C(42)-C(41)-C(46)$ & $119.38(17)$ \\
\hline $\mathrm{C}(42)-\mathrm{C}(41)-\mathrm{S}(2)$ & $121.49(14)$ \\
\hline $\mathrm{C}(46)-\mathrm{C}(41)-\mathrm{S}(2)$ & $118.89(14)$ \\
\hline$C(41)-C(42)-C(43)$ & $120.00(19)$ \\
\hline$C(44)-C(43)-C(42)$ & $120.3(2)$ \\
\hline$C(43)-C(44)-C(45)$ & $119.9(2)$ \\
\hline$C(46)-C(45)-C(44)$ & $119.9(2)$ \\
\hline $\mathrm{C}(45)-\mathrm{C}(46)-\mathrm{C}(41)$ & $120.53(19)$ \\
\hline $\mathrm{C}(48)-\mathrm{C}(47)-\mathrm{C}(52)$ & $117.63(16)$ \\
\hline $\mathrm{C}(48)-\mathrm{C}(47)-\mathrm{C}(32)$ & $121.61(15)$ \\
\hline $\mathrm{C}(52)-\mathrm{C}(47)-\mathrm{C}(32)$ & $120.75(15)$ \\
\hline $\mathrm{C}(47)-\mathrm{C}(48)-\mathrm{C}(49)$ & $120.80(17)$ \\
\hline$C(50)-C(49)-C(48)$ & $120.72(18)$ \\
\hline $\mathrm{C}(49)-\mathrm{C}(50)-\mathrm{C}(51)$ & $119.45(17)$ \\
\hline $\mathrm{C}(50)-\mathrm{C}(51)-\mathrm{C}(52)$ & $119.98(17)$ \\
\hline $\mathrm{C}(51)-\mathrm{C}(52)-\mathrm{C}(47)$ & $121.41(17)$ \\
\hline
\end{tabular}


Table 4. Anisotropic displacement parameters $\left(\AA^{2} \times 10^{3}\right)$ for kaw82. The anisotropic displacement factor exponent takes the form: $-2 \square^{2}\left[h^{2} a^{* 2} U^{11}+\ldots+2 h \mathrm{k} \mathrm{a}^{*} \mathrm{~b}^{*} \mathrm{U}^{12}\right]$

\begin{tabular}{|c|c|c|c|c|c|c|}
\hline & $\mathrm{U}^{11}$ & $\mathrm{U}^{22}$ & $\mathrm{U}^{33}$ & $\mathrm{U}^{23}$ & $\mathrm{U}^{13}$ & $\mathrm{U}^{12}$ \\
\hline$S(1)$ & $18(1)$ & $24(1)$ & $31(1)$ & $-4(1)$ & $0(1)$ & $-1(1)$ \\
\hline $\mathrm{O}(1)$ & $26(1)$ & $26(1)$ & $20(1)$ & $-2(1)$ & $5(1)$ & $-4(1)$ \\
\hline$C(1)$ & $16(1)$ & $20(1)$ & $21(1)$ & $0(1)$ & $0(1)$ & $0(1)$ \\
\hline$C(2)$ & $19(1)$ & $23(1)$ & $16(1)$ & $2(1)$ & $1(1)$ & $1(1)$ \\
\hline$C(3)$ & $22(1)$ & $27(1)$ & $21(1)$ & $3(1)$ & $-3(1)$ & $-3(1)$ \\
\hline$C(4)$ & $26(1)$ & $27(1)$ & $23(1)$ & $5(1)$ & $-4(1)$ & $-7(1)$ \\
\hline$C(5)$ & $24(1)$ & $27(1)$ & $20(1)$ & $6(1)$ & $0(1)$ & $-3(1)$ \\
\hline$C(6)$ & $22(1)$ & $22(1)$ & $18(1)$ & $1(1)$ & $-1(1)$ & $2(1)$ \\
\hline$C(7)$ & $44(1)$ & $24(1)$ & $27(1)$ & $2(1)$ & $-6(1)$ & $-5(1)$ \\
\hline$C(8)$ & $32(1)$ & $45(1)$ & $37(1)$ & $14(1)$ & $-7(1)$ & $-18(1)$ \\
\hline $\mathrm{C}(9)$ & $25(1)$ & $22(1)$ & $34(1)$ & $2(1)$ & $1(1)$ & $4(1)$ \\
\hline$C(10)$ & $33(1)$ & $35(1)$ & $21(1)$ & $1(1)$ & $-5(1)$ & $6(1)$ \\
\hline $\mathrm{C}(11)$ & $24(1)$ & $24(1)$ & $29(1)$ & $-4(1)$ & $-8(1)$ & $0(1)$ \\
\hline $\mathrm{C}(12)$ & $58(1)$ & $32(1)$ & $33(1)$ & $-1(1)$ & $2(1)$ & $-6(1)$ \\
\hline$C(13)$ & $101(2)$ & $26(1)$ & $49(2)$ & $6(1)$ & $0(2)$ & $-9(1)$ \\
\hline$C(14)$ & $82(2)$ & $27(1)$ & $51(2)$ & $-9(1)$ & $-8(1)$ & $9(1)$ \\
\hline$C(15)$ & $46(1)$ & $39(1)$ & $41(1)$ & $-14(1)$ & $-3(1)$ & $8(1)$ \\
\hline$C(16)$ & $31(1)$ & $30(1)$ & $34(1)$ & $-4(1)$ & $-4(1)$ & $-2(1)$ \\
\hline$C(17)$ & $24(1)$ & $23(1)$ & $22(1)$ & $2(1)$ & $-6(1)$ & $-3(1)$ \\
\hline$C(18)$ & $25(1)$ & $28(1)$ & $28(1)$ & $3(1)$ & $-5(1)$ & $-7(1)$ \\
\hline$C(19)$ & $40(1)$ & $29(1)$ & $42(1)$ & 11(1) & $-13(1)$ & $-12(1)$ \\
\hline $\mathrm{C}(20)$ & $52(1)$ & $21(1)$ & $53(1)$ & $0(1)$ & $-26(1)$ & 1(1) \\
\hline $\mathrm{C}(21)$ & $44(1)$ & $35(1)$ & $40(1)$ & $-7(1)$ & $-10(1)$ & $14(1)$ \\
\hline $\mathrm{C}(22)$ & $34(1)$ & $32(1)$ & $27(1)$ & $0(1)$ & $-5(1)$ & $5(1)$ \\
\hline$S(2)$ & $23(1)$ & $21(1)$ & $18(1)$ & $-1(1)$ & $0(1)$ & $2(1)$ \\
\hline $\mathrm{O}(2)$ & 21(1) & $21(1)$ & $20(1)$ & $0(1)$ & $-3(1)$ & $2(1)$ \\
\hline$C(31)$ & $18(1)$ & $19(1)$ & $18(1)$ & $-1(1)$ & $1(1)$ & $-1(1)$ \\
\hline$C(32)$ & $18(1)$ & $20(1)$ & $19(1)$ & $1(1)$ & $-1(1)$ & $0(1)$ \\
\hline$C(33)$ & $21(1)$ & $21(1)$ & $21(1)$ & $1(1)$ & $2(1)$ & $1(1)$ \\
\hline$C(34)$ & $22(1)$ & $19(1)$ & $22(1)$ & $-1(1)$ & $0(1)$ & $0(1)$ \\
\hline$C(35)$ & $22(1)$ & $20(1)$ & $24(1)$ & $0(1)$ & $0(1)$ & $-3(1)$ \\
\hline
\end{tabular}




\begin{tabular}{lllllll}
$\mathrm{C}(36)$ & $20(1)$ & $21(1)$ & $25(1)$ & $0(1)$ & $3(1)$ & $-3(1)$ \\
$\mathrm{C}(37)$ & $34(1)$ & $26(1)$ & $25(1)$ & $-4(1)$ & $2(1)$ & $1(1)$ \\
$\mathrm{C}(38)$ & $28(1)$ & $22(1)$ & $30(1)$ & $1(1)$ & $-2(1)$ & $3(1)$ \\
$\mathrm{C}(39)$ & $25(1)$ & $29(1)$ & $40(1)$ & $-5(1)$ & $12(1)$ & $-9(1)$ \\
$\mathrm{C}(40)$ & $41(1)$ & $23(1)$ & $22(1)$ & $5(1)$ & $5(1)$ & $-3(1)$ \\
$\mathrm{C}(41)$ & $23(1)$ & $22(1)$ & $18(1)$ & $-1(1)$ & $4(1)$ & $4(1)$ \\
$\mathrm{C}(42)$ & $25(1)$ & $29(1)$ & $30(1)$ & $-6(1)$ & $0(1)$ & $3(1)$ \\
$\mathrm{C}(43)$ & $32(1)$ & $47(1)$ & $33(1)$ & $-5(1)$ & $-9(1)$ & $15(1)$ \\
$\mathrm{C}(44)$ & $55(1)$ & $31(1)$ & $37(1)$ & $5(1)$ & $-3(1)$ & $20(1)$ \\
$\mathrm{C}(45)$ & $50(1)$ & $25(1)$ & $51(1)$ & $0(1)$ & $-7(1)$ & $1(1)$ \\
$\mathrm{C}(46)$ & $28(1)$ & $29(1)$ & $37(1)$ & $0(1)$ & $-4(1)$ & $-1(1)$ \\
$\mathrm{C}(47)$ & $19(1)$ & $21(1)$ & $17(1)$ & $1(1)$ & $3(1)$ & $1(1)$ \\
$\mathrm{C}(48)$ & $22(1)$ & $23(1)$ & $20(1)$ & $1(1)$ & $1(1)$ & $0(1)$ \\
$\mathrm{C}(49)$ & $27(1)$ & $26(1)$ & $28(1)$ & $-2(1)$ & $3(1)$ & $-6(1)$ \\
$\mathrm{C}(50)$ & $34(1)$ & $20(1)$ & $31(1)$ & $6(1)$ & $7(1)$ & $-2(1)$ \\
$\mathrm{C}(51)$ & $29(1)$ & $27(1)$ & $30(1)$ & $10(1)$ & $-1(1)$ & $1(1)$ \\
$\mathrm{C}(52)$ & $22(1)$ & $27(1)$ & $27(1)$ & $6(1)$ & $-2(1)$ & $-3(1)$ \\
& & & & & & \\
\hline
\end{tabular}


Table 5. Hydrogen coordinates ( $\left.\times 10^{4}\right)$ and isotropic displacement parameters $\left(\AA^{2} \times 10^{3}\right)$ for kaw82.

\begin{tabular}{|c|c|c|c|c|}
\hline & $\mathrm{x}$ & $\mathrm{y}$ & z & $\mathrm{U}(\mathrm{eq})$ \\
\hline $\mathrm{H}(1)$ & $8586(15)$ & $960(20)$ & $4926(13)$ & $42(7)$ \\
\hline $\mathrm{H}(1 \mathrm{~A})$ & $7794(12)$ & $1532(18)$ & $6393(10)$ & $25(5)$ \\
\hline $\mathrm{H}(3 \mathrm{~A})$ & $6529(11)$ & $616(17)$ & $5708(9)$ & $16(4)$ \\
\hline $\mathrm{H}(3 \mathrm{~B})$ & $6708(11)$ & $198(17)$ & $5010(10)$ & $19(5)$ \\
\hline $\mathrm{H}(5 \mathrm{~A})$ & $7644(12)$ & $-1771(19)$ & $6518(10)$ & $26(5)$ \\
\hline $\mathrm{H}(5 \mathrm{~B})$ & $7153(11)$ & $-521(17)$ & $6651(9)$ & $16(5)$ \\
\hline $\mathrm{H}(7 \mathrm{~A})$ & $8040(13)$ & $-1545(19)$ & $5070(10)$ & $28(5)$ \\
\hline $\mathrm{H}(7 \mathrm{~B})$ & $7163(14)$ & $-1956(19)$ & $4814(11)$ & $36(6)$ \\
\hline $\mathrm{H}(7 \mathrm{C})$ & $7572(13)$ & $-2710(20)$ & $5411(11)$ & $32(6)$ \\
\hline $\mathrm{H}(8 \mathrm{~A})$ & $5937(15)$ & $-1100(20)$ & $6143(13)$ & $46(7)$ \\
\hline $\mathrm{H}(8 \mathrm{~B})$ & $5937(15)$ & $-1590(20)$ & $5419(12)$ & $42(7)$ \\
\hline $\mathrm{H}(8 \mathrm{C})$ & $6293(14)$ & $-2390(20)$ & $6013(13)$ & $42(6)$ \\
\hline $\mathrm{H}(9 \mathrm{~A})$ & $9057(13)$ & $-1640(20)$ & $6258(11)$ & $33(6)$ \\
\hline H(9B) & $9493(13)$ & $-410(20)$ & $6184(11)$ & $33(6)$ \\
\hline $\mathrm{H}(9 \mathrm{C})$ & $8972(13)$ & $-910(20)$ & $5578(12)$ & $32(6)$ \\
\hline $\mathrm{H}(10 \mathrm{~A})$ & $8020(14)$ & $370(20)$ & $7319(11)$ & $35(6)$ \\
\hline $\mathrm{H}(10 \mathrm{~B})$ & $8998(14)$ & $570(20)$ & $7162(11)$ & $37(6)$ \\
\hline $\mathrm{H}(10 \mathrm{C})$ & $8641(14)$ & $-760(20)$ & $7322(12)$ & $43(7)$ \\
\hline $\mathrm{H}(12 \mathrm{~A})$ & $9382(14)$ & $4230(20)$ & $5562(13)$ & $37(6)$ \\
\hline $\mathrm{H}(13)$ & $9248(19)$ & $6150(30)$ & $5905(16)$ & $76(10)$ \\
\hline $\mathrm{H}(14)$ & $8589(16)$ & $6500(30)$ & $6845(14)$ & $60(8)$ \\
\hline $\mathrm{H}(15)$ & $8023(16)$ & $4940(20)$ & $7449(13)$ & $51(7)$ \\
\hline $\mathrm{H}(16)$ & $8212(14)$ & $2960(20)$ & $7097(12)$ & $38(6)$ \\
\hline $\mathrm{H}(18)$ & $8091(12)$ & $2648(18)$ & $4471(10)$ & $25(5)$ \\
\hline $\mathrm{H}(19)$ & $7698(12)$ & 4651(19) & $4292(11)$ & $30(5)$ \\
\hline $\mathrm{H}(20)$ & $6797(17)$ & $5580(30)$ & $4974(14)$ & $65(9)$ \\
\hline $\mathrm{H}(21)$ & $6238(15)$ & $4530(20)$ & $5836(12)$ & $46(7)$ \\
\hline $\mathrm{H}(22)$ & $6618(12)$ & $2515(19)$ & $6062(11)$ & $31(5)$ \\
\hline $\mathrm{H}(2)$ & $6568(12)$ & $-647(19)$ & $9084(11)$ & $24(5)$ \\
\hline $\mathrm{H}(31 \mathrm{~A})$ & $4790(12)$ & $-562(17)$ & $8273(10)$ & $20(5)$ \\
\hline
\end{tabular}




\begin{tabular}{|c|c|c|c|c|}
\hline $\mathrm{H}(33 \mathrm{~A})$ & $5846(11)$ & $-1438(18)$ & $7408(10)$ & $22(5)$ \\
\hline $\mathrm{H}(33 \mathrm{~B})$ & $6677(12)$ & $-1762(17)$ & $7657(10)$ & $23(5)$ \\
\hline $\mathrm{H}(35 \mathrm{~A})$ & $4835(12)$ & $-3818(18)$ & $8295(10)$ & $19(5)$ \\
\hline $\mathrm{H}(35 \mathrm{~B})$ & $4656(12)$ & $-2739(17)$ & $7822(9)$ & $19(5)$ \\
\hline $\mathrm{H}(37 \mathrm{~A})$ & $5625(14)$ & $-4520(20)$ & $7315(11)$ & $37(6)$ \\
\hline $\mathrm{H}(37 \mathrm{~B})$ & $5420(14)$ & $-3310(20)$ & $6962(12)$ & $37(6)$ \\
\hline $\mathrm{H}(37 \mathrm{C})$ & $6314(16)$ & $-3800(20)$ & $7065(12)$ & $48(7)$ \\
\hline $\mathrm{H}(38 \mathrm{~A})$ & $6596(13)$ & $-3367(19)$ & $8693(11)$ & $29(5)$ \\
\hline $\mathrm{H}(38 \mathrm{~B})$ & $6212(14)$ & $-4550(20)$ & $8467(11)$ & $37(6)$ \\
\hline $\mathrm{H}(38 \mathrm{C})$ & $6968(14)$ & $-3910(20)$ & $8088(11)$ & $35(6)$ \\
\hline $\mathrm{H}(39 \mathrm{~A})$ & $3750(13)$ & $-2900(20)$ & $8929(10)$ & $28(5)$ \\
\hline $\mathrm{H}(39 \mathrm{~B})$ & $3683(13)$ & $-1832(19)$ & $8468(11)$ & $30(6)$ \\
\hline $\mathrm{H}(39 \mathrm{C})$ & $3800(14)$ & $-1610(20)$ & $9221(13)$ & $48(7)$ \\
\hline $\mathrm{H}(40 \mathrm{~A})$ & $5045(13)$ & $-3560(20)$ & $9466(12)$ & $37(6)$ \\
\hline $\mathrm{H}(40 \mathrm{~B})$ & $5007(12)$ & $-2250(20)$ & $9779(11)$ & $25(5)$ \\
\hline $\mathrm{H}(40 \mathrm{C})$ & $5794(15)$ & $-2660(20)$ & $9410(11)$ & $38(6)$ \\
\hline $\mathrm{H}(42)$ & $3616(14)$ & $690(20)$ & $8649(11)$ & $37(6)$ \\
\hline $\mathrm{H}(43)$ & $3097(16)$ & $2430(20)$ & $8317(13)$ & $47(7)$ \\
\hline $\mathrm{H}(44)$ & $3628(15)$ & $4270(20)$ & $8508(12)$ & $44(7)$ \\
\hline $\mathrm{H}(45)$ & $4903(13)$ & $4320(20)$ & $9002(12)$ & $41(6)$ \\
\hline $\mathrm{H}(46)$ & $5478(15)$ & $2570(20)$ & $9288(12)$ & $36(6)$ \\
\hline $\mathrm{H}(48)$ & $7123(10)$ & $863(16)$ & $8577(9)$ & $11(4)$ \\
\hline $\mathrm{H}(49)$ & $7312(14)$ & $2800(20)$ & $8162(11)$ & $33(6)$ \\
\hline $\mathrm{H}(50)$ & $6483(13)$ & $3580(20)$ & $7353(11)$ & $33(6)$ \\
\hline $\mathrm{H}(51)$ & $5418(13)$ & $2354(19)$ & $6971(10)$ & $28(5)$ \\
\hline $\mathrm{H}(52)$ & $5225(14)$ & $460(20)$ & $7398(11)$ & $33(6)$ \\
\hline
\end{tabular}


Table 6. Torsion angles $\left[{ }^{\circ}\right]$ for kaw82.

\begin{tabular}{|c|c|}
\hline$C(11)-S(1)-C(1)-C(6)$ & $-136.50(12)$ \\
\hline $\mathrm{C}(11)-\mathrm{S}(1)-\mathrm{C}(1)-\mathrm{C}(2)$ & $94.48(13)$ \\
\hline $\mathrm{C}(6)-\mathrm{C}(1)-\mathrm{C}(2)-\mathrm{O}(1)$ & $-70.61(18)$ \\
\hline $\mathrm{S}(1)-\mathrm{C}(1)-\mathrm{C}(2)-\mathrm{O}(1)$ & $54.39(16)$ \\
\hline $\mathrm{C}(6)-\mathrm{C}(1)-\mathrm{C}(2)-\mathrm{C}(17)$ & $167.50(14)$ \\
\hline$S(1)-C(1)-C(2)-C(17)$ & $-67.50(14)$ \\
\hline $\mathrm{C}(6)-\mathrm{C}(1)-\mathrm{C}(2)-\mathrm{C}(3)$ & $46.50(19)$ \\
\hline $\mathrm{S}(1)-\mathrm{C}(1)-\mathrm{C}(2)-\mathrm{C}(3)$ & $171.50(12)$ \\
\hline $\mathrm{O}(1)-\mathrm{C}(2)-\mathrm{C}(3)-\mathrm{C}(4)$ & $72.15(18)$ \\
\hline$C(17)-C(2)-C(3)-C(4)$ & $-168.33(15)$ \\
\hline $\mathrm{C}(1)-\mathrm{C}(2)-\mathrm{C}(3)-\mathrm{C}(4)$ & $-48.2(2)$ \\
\hline$C(2)-C(3)-C(4)-C(7)$ & $-75.8(2)$ \\
\hline$C(2)-C(3)-C(4)-C(5)$ & $50.2(2)$ \\
\hline $\mathrm{C}(2)-\mathrm{C}(3)-\mathrm{C}(4)-\mathrm{C}(8)$ & $166.36(16)$ \\
\hline$C(7)-C(4)-C(5)-C(6)$ & $72.8(2)$ \\
\hline$C(3)-C(4)-C(5)-C(6)$ & $-53.4(2)$ \\
\hline$C(8)-C(4)-C(5)-C(6)$ & $-169.03(18)$ \\
\hline $\mathrm{C}(4)-\mathrm{C}(5)-\mathrm{C}(6)-\mathrm{C}(10)$ & $168.40(16)$ \\
\hline$C(4)-C(5)-C(6)-C(9)$ & $-73.9(2)$ \\
\hline$C(4)-C(5)-C(6)-C(1)$ & $52.3(2)$ \\
\hline $\mathrm{C}(2)-\mathrm{C}(1)-\mathrm{C}(6)-\mathrm{C}(10)$ & $-162.30(14)$ \\
\hline$S(1)-C(1)-C(6)-C(10)$ & $72.67(16)$ \\
\hline $\mathrm{C}(2)-\mathrm{C}(1)-\mathrm{C}(6)-\mathrm{C}(9)$ & $78.97(18)$ \\
\hline$S(1)-C(1)-C(6)-C(9)$ & $-46.06(17)$ \\
\hline$C(2)-C(1)-C(6)-C(5)$ & $-47.32(18)$ \\
\hline$S(1)-C(1)-C(6)-C(5)$ & $-172.35(11)$ \\
\hline$C(1)-S(1)-C(11)-C(16)$ & $48.75(17)$ \\
\hline $\mathrm{C}(1)-\mathrm{S}(1)-\mathrm{C}(11)-\mathrm{C}(12)$ & $-137.68(16)$ \\
\hline$C(16)-C(11)-C(12)-C(13)$ & $0.6(3)$ \\
\hline $\mathrm{S}(1)-\mathrm{C}(11)-\mathrm{C}(12)-\mathrm{C}(13)$ & $-173.1(2)$ \\
\hline $\mathrm{C}(11)-\mathrm{C}(12)-\mathrm{C}(13)-\mathrm{C}(14)$ & $-1.0(4)$ \\
\hline $\mathrm{C}(12)-\mathrm{C}(13)-\mathrm{C}(14)-\mathrm{C}(15)$ & $-0.3(4)$ \\
\hline$C(13)-C(14)-C(15)-C(16)$ & $2.1(4)$ \\
\hline$C(12)-C(11)-C(16)-C(15)$ & $1.1(3)$ \\
\hline
\end{tabular}


$\mathrm{S}(1)-\mathrm{C}(11)-\mathrm{C}(16)-\mathrm{C}(15)$

$\mathrm{C}(14)-\mathrm{C}(15)-\mathrm{C}(16)-\mathrm{C}(11)$

$\mathrm{O}(1)-\mathrm{C}(2)-\mathrm{C}(17)-\mathrm{C}(18)$

$\mathrm{C}(3)-\mathrm{C}(2)-\mathrm{C}(17)-\mathrm{C}(18)$

$\mathrm{C}(1)-\mathrm{C}(2)-\mathrm{C}(17)-\mathrm{C}(18)$

$\mathrm{O}(1)-\mathrm{C}(2)-\mathrm{C}(17)-\mathrm{C}(22)$

$\mathrm{C}(3)-\mathrm{C}(2)-\mathrm{C}(17)-\mathrm{C}(22)$

$\mathrm{C}(1)-\mathrm{C}(2)-\mathrm{C}(17)-\mathrm{C}(22)$

$\mathrm{C}(22)-\mathrm{C}(17)-\mathrm{C}(18)-\mathrm{C}(19)$

$\mathrm{C}(2)-\mathrm{C}(17)-\mathrm{C}(18)-\mathrm{C}(19)$

$\mathrm{C}(17)-\mathrm{C}(18)-\mathrm{C}(19)-\mathrm{C}(20)$

$\mathrm{C}(18)-\mathrm{C}(19)-\mathrm{C}(20)-\mathrm{C}(21)$

$\mathrm{C}(19)-\mathrm{C}(20)-\mathrm{C}(21)-\mathrm{C}(22)$

$\mathrm{C}(20)-\mathrm{C}(21)-\mathrm{C}(22)-\mathrm{C}(17)$

$\mathrm{C}(18)-\mathrm{C}(17)-\mathrm{C}(22)-\mathrm{C}(21)$

$\mathrm{C}(2)-\mathrm{C}(17)-\mathrm{C}(22)-\mathrm{C}(21)$

$\mathrm{C}(41)-\mathrm{S}(2)-\mathrm{C}(31)-\mathrm{C}(36)$

$\mathrm{C}(41)-\mathrm{S}(2)-\mathrm{C}(31)-\mathrm{C}(32)$

$\mathrm{C}(36)-\mathrm{C}(31)-\mathrm{C}(32)-\mathrm{O}(2)$

$\mathrm{S}(2)-\mathrm{C}(31)-\mathrm{C}(32)-\mathrm{O}(2)$

$\mathrm{C}(36)-\mathrm{C}(31)-\mathrm{C}(32)-\mathrm{C}(47)$

$\mathrm{S}(2)-\mathrm{C}(31)-\mathrm{C}(32)-\mathrm{C}(47)$

$\mathrm{C}(36)-\mathrm{C}(31)-\mathrm{C}(32)-\mathrm{C}(33)$

$\mathrm{S}(2)-\mathrm{C}(31)-\mathrm{C}(32)-\mathrm{C}(33)$

$\mathrm{O}(2)-\mathrm{C}(32)-\mathrm{C}(33)-\mathrm{C}(34)$

$\mathrm{C}(47)-\mathrm{C}(32)-\mathrm{C}(33)-\mathrm{C}(34)$

$\mathrm{C}(31)-\mathrm{C}(32)-\mathrm{C}(33)-\mathrm{C}(34)$

$\mathrm{C}(32)-\mathrm{C}(33)-\mathrm{C}(34)-\mathrm{C}(35)$

$\mathrm{C}(32)-\mathrm{C}(33)-\mathrm{C}(34)-\mathrm{C}(38)$

$\mathrm{C}(32)-\mathrm{C}(33)-\mathrm{C}(34)-\mathrm{C}(37)$

C(38)-C(34)-C(35)-C(36)

$\mathrm{C}(37)-\mathrm{C}(34)-\mathrm{C}(35)-\mathrm{C}(36)$

C(33)-C(34)-C(35)-C(36)

$\mathrm{C}(34)-\mathrm{C}(35)-\mathrm{C}(36)-\mathrm{C}(39)$

$\mathrm{C}(34)-\mathrm{C}(35)-\mathrm{C}(36)-\mathrm{C}(40)$

$\mathrm{C}(34)-\mathrm{C}(35)-\mathrm{C}(36)-\mathrm{C}(31)$
174.60(16)

$-2.5(3)$

$-7.8(2)$

$-124.07(17)$

114.23(17)

173.67(16)

$57.4(2)$

$-64.3(2)$

$0.6(3)$

$-178.01(17)$

$-1.2(3)$

$0.6(3)$

$0.4(3)$

$-1.0(3)$

$0.5(3)$

179.10(17)

$-132.98(12)$

97.88(12)

$-68.96(18)$

$56.87(16)$

167.90(14)

$-66.27(15)$

48.62(18)

174.46(11)

71.43(18)

$-169.20(14)$

$-49.29(19)$

49.65(19)

$-76.31(19)$

165.16(15)

73.3(2)

$-168.42(15)$

$-52.4(2)$

168.43(16)

$-73.3(2)$

52.6(2) 


\begin{tabular}{|c|c|}
\hline $\mathrm{C}(32)-\mathrm{C}(31)-\mathrm{C}(36)-\mathrm{C}(39)$ & $-163.74(15)$ \\
\hline $\mathrm{S}(2)-\mathrm{C}(31)-\mathrm{C}(36)-\mathrm{C}(39)$ & $70.19(16)$ \\
\hline $\mathrm{C}(32)-\mathrm{C}(31)-\mathrm{C}(36)-\mathrm{C}(40)$ & $77.07(18)$ \\
\hline $\mathrm{S}(2)-\mathrm{C}(31)-\mathrm{C}(36)-\mathrm{C}(40)$ & $-49.00(17)$ \\
\hline $\mathrm{C}(32)-\mathrm{C}(31)-\mathrm{C}(36)-\mathrm{C}(35)$ & $-49.09(18)$ \\
\hline $\mathrm{S}(2)-\mathrm{C}(31)-\mathrm{C}(36)-\mathrm{C}(35)$ & $-175.17(11)$ \\
\hline $\mathrm{C}(31)-\mathrm{S}(2)-\mathrm{C}(41)-\mathrm{C}(42)$ & $59.28(16)$ \\
\hline $\mathrm{C}(31)-\mathrm{S}(2)-\mathrm{C}(41)-\mathrm{C}(46)$ & $-126.29(15)$ \\
\hline $\mathrm{C}(46)-\mathrm{C}(41)-\mathrm{C}(42)-\mathrm{C}(43)$ & $1.1(3)$ \\
\hline$S(2)-C(41)-C(42)-C(43)$ & $175.47(15)$ \\
\hline $\mathrm{C}(41)-\mathrm{C}(42)-\mathrm{C}(43)-\mathrm{C}(44)$ & $-1.0(3)$ \\
\hline $\mathrm{C}(42)-\mathrm{C}(43)-\mathrm{C}(44)-\mathrm{C}(45)$ & $-0.2(3)$ \\
\hline $\mathrm{C}(43)-\mathrm{C}(44)-\mathrm{C}(45)-\mathrm{C}(46)$ & $1.2(4)$ \\
\hline $\mathrm{C}(44)-\mathrm{C}(45)-\mathrm{C}(46)-\mathrm{C}(41)$ & $-1.1(3)$ \\
\hline $\mathrm{C}(42)-\mathrm{C}(41)-\mathrm{C}(46)-\mathrm{C}(45)$ & $-0.1(3)$ \\
\hline $\mathrm{S}(2)-\mathrm{C}(41)-\mathrm{C}(46)-\mathrm{C}(45)$ & $-174.60(17)$ \\
\hline $\mathrm{O}(2)-\mathrm{C}(32)-\mathrm{C}(47)-\mathrm{C}(48)$ & $-1.1(2)$ \\
\hline $\mathrm{C}(33)-\mathrm{C}(32)-\mathrm{C}(47)-\mathrm{C}(48)$ & $-117.43(17)$ \\
\hline $\mathrm{C}(31)-\mathrm{C}(32)-\mathrm{C}(47)-\mathrm{C}(48)$ & $122.22(17)$ \\
\hline $\mathrm{O}(2)-\mathrm{C}(32)-\mathrm{C}(47)-\mathrm{C}(52)$ & $178.57(15)$ \\
\hline$C(33)-C(32)-C(47)-C(52)$ & $62.3(2)$ \\
\hline $\mathrm{C}(31)-\mathrm{C}(32)-\mathrm{C}(47)-\mathrm{C}(52)$ & $-58.1(2)$ \\
\hline$C(52)-C(47)-C(48)-C(49)$ & $0.9(3)$ \\
\hline $\mathrm{C}(32)-\mathrm{C}(47)-\mathrm{C}(48)-\mathrm{C}(49)$ & $-179.44(16)$ \\
\hline$C(47)-C(48)-C(49)-C(50)$ & $-0.6(3)$ \\
\hline $\mathrm{C}(48)-\mathrm{C}(49)-\mathrm{C}(50)-\mathrm{C}(51)$ & $0.3(3)$ \\
\hline$C(49)-C(50)-C(51)-C(52)$ & $-0.3(3)$ \\
\hline $\mathrm{C}(50)-\mathrm{C}(51)-\mathrm{C}(52)-\mathrm{C}(47)$ & $0.6(3)$ \\
\hline $\mathrm{C}(48)-\mathrm{C}(47)-\mathrm{C}(52)-\mathrm{C}(51)$ & $-0.9(3)$ \\
\hline $\mathrm{C}(32)-\mathrm{C}(47)-\mathrm{C}(52)-\mathrm{C}(51)$ & $179.40(16)$ \\
\hline
\end{tabular}


Supporting

B. GC traces illustrating high diastereoselectivity:

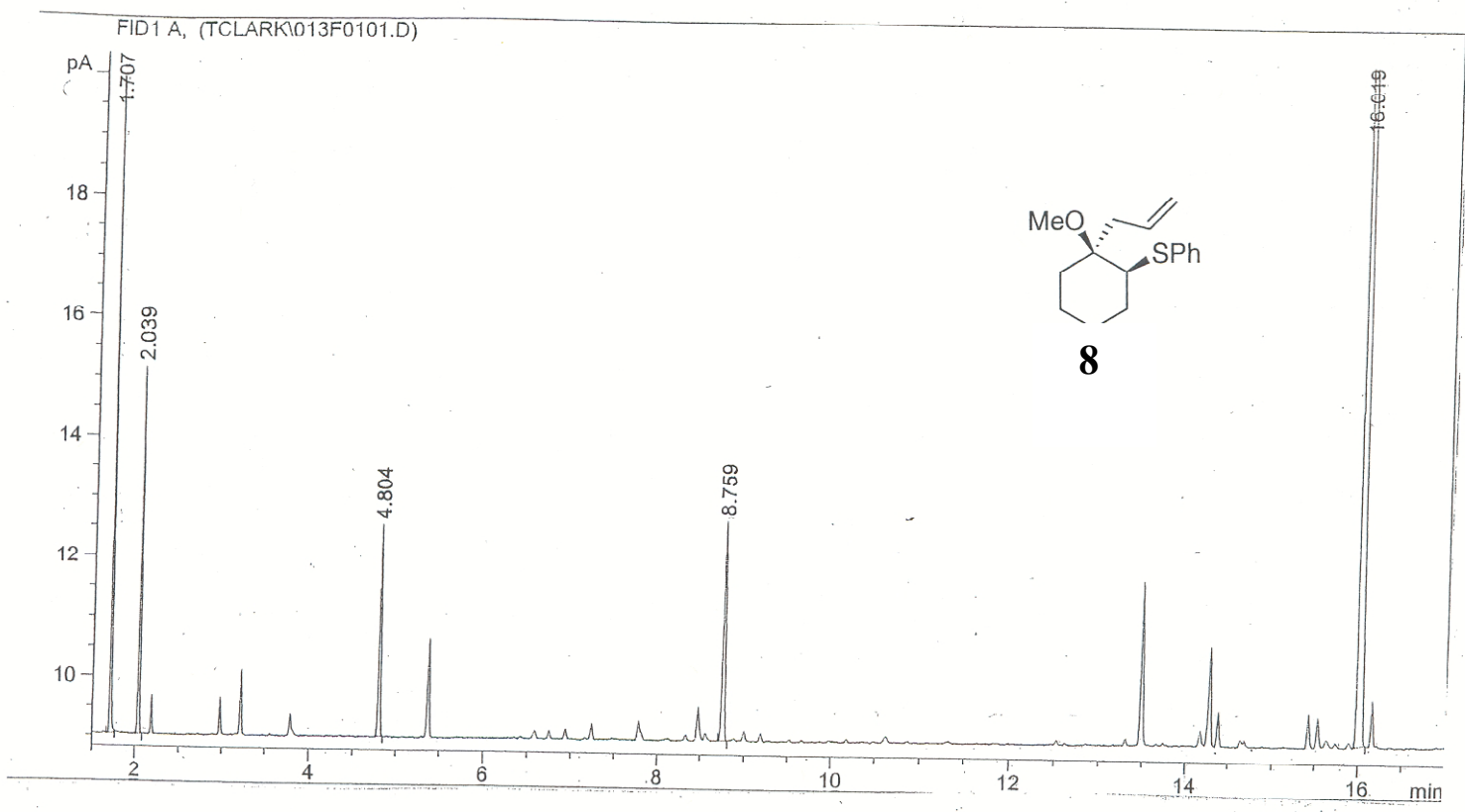




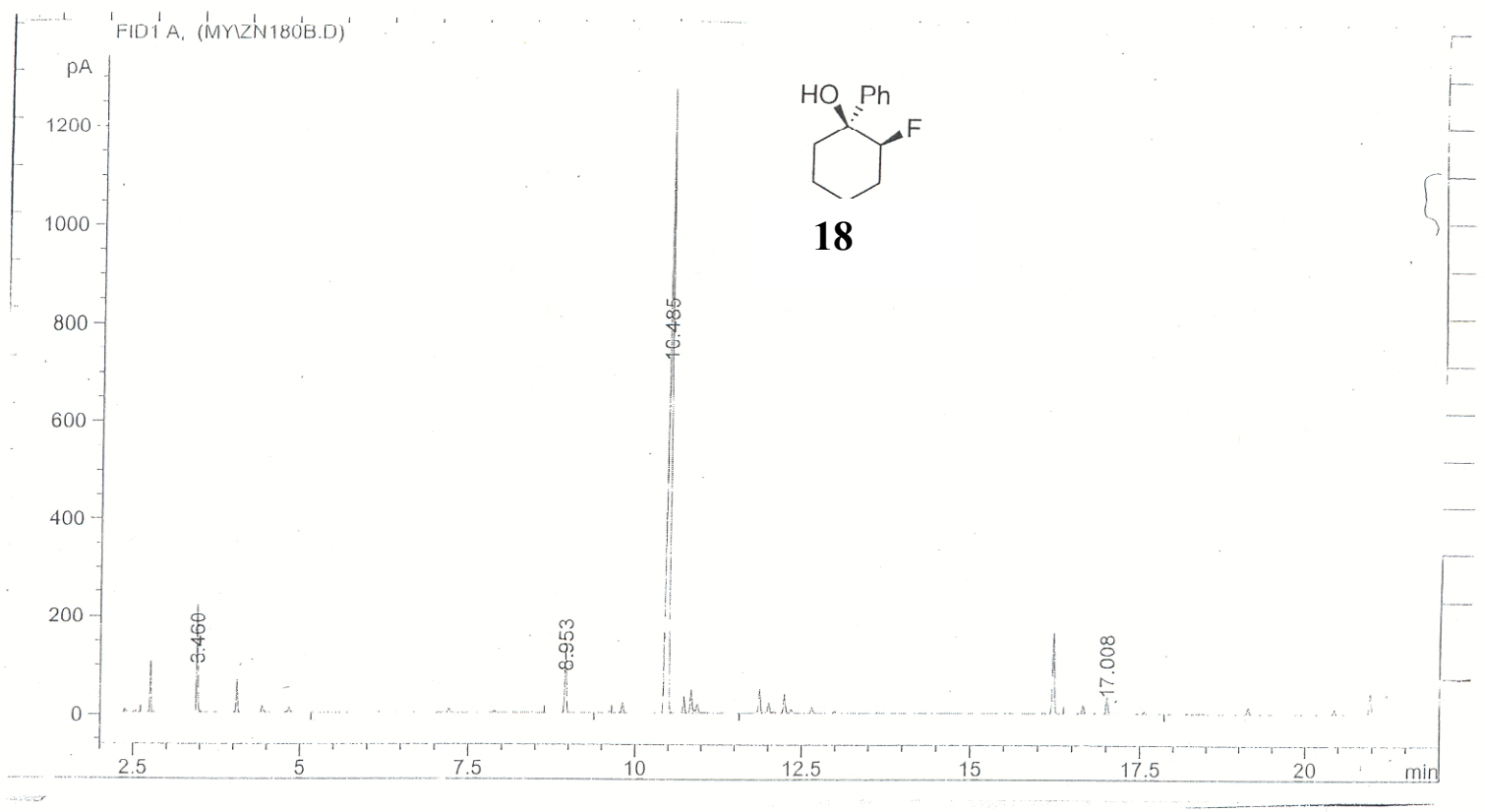




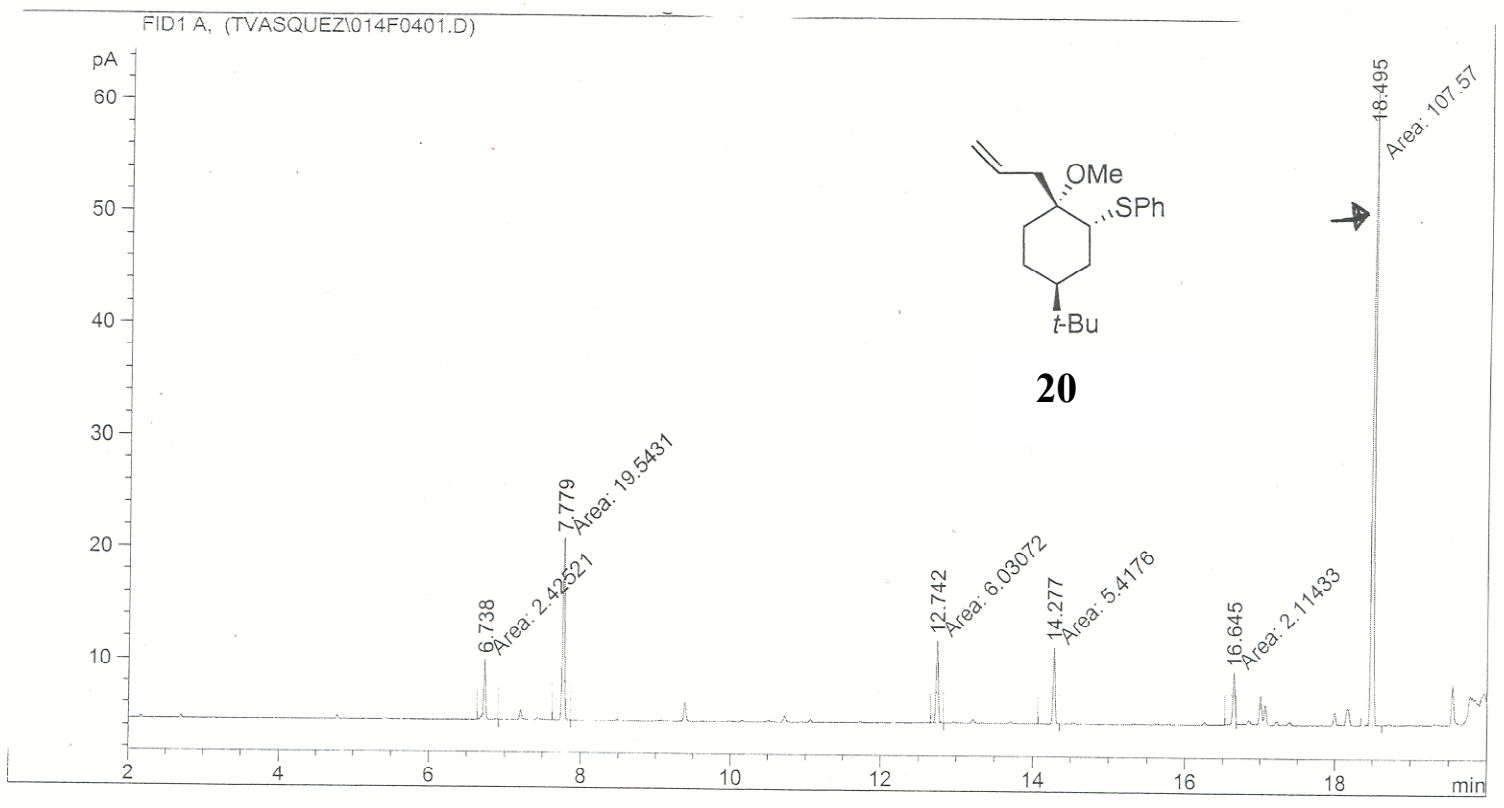

\begin{tabular}{|c|c|c|c|c|c|c|}
\hline $\begin{array}{c}\text { eak } \\
\#\end{array}$ & $\begin{array}{c}\text { RetTime } \\
\text { [min] }\end{array}$ & Type & $\begin{array}{l}\text { Width } \\
\text { [min] }\end{array}$ & $\begin{array}{r}\text { Area } \\
{\left[\mathrm{pA}^{*} \mathrm{~S}\right]}\end{array}$ & $\begin{array}{l}\text { Height } \\
{[p A]}\end{array}$ & $\begin{array}{c}\text { Area } \\
\frac{\circ}{\circ}\end{array}$ \\
\hline 1 & 6.738 & MM & 0.0156 & 2.42521 & 2.58748 & 1.69476 \\
\hline 2 & 7.779 & MM & 0.0254 & 19.54313 & 12.81738 & 13.65691 \\
\hline 3 & 12. & MM & 0.0217 & 6.03072 & 4.62 & 4. \\
\hline 4 & 14.2 & MM & 0.0209 & 5.417 & 4. & 3. \\
\hline & 16.645 & $\mathrm{MM}$ & 0.0174 & 2.114 & 2.02 & 1. \\
\hline & 18.495 & MM & 0.0327 & 107.56966 & 54.88038 & 75.17063 \\
\hline
\end{tabular}




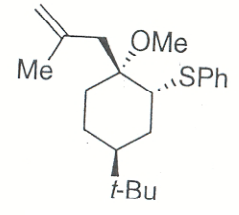

21

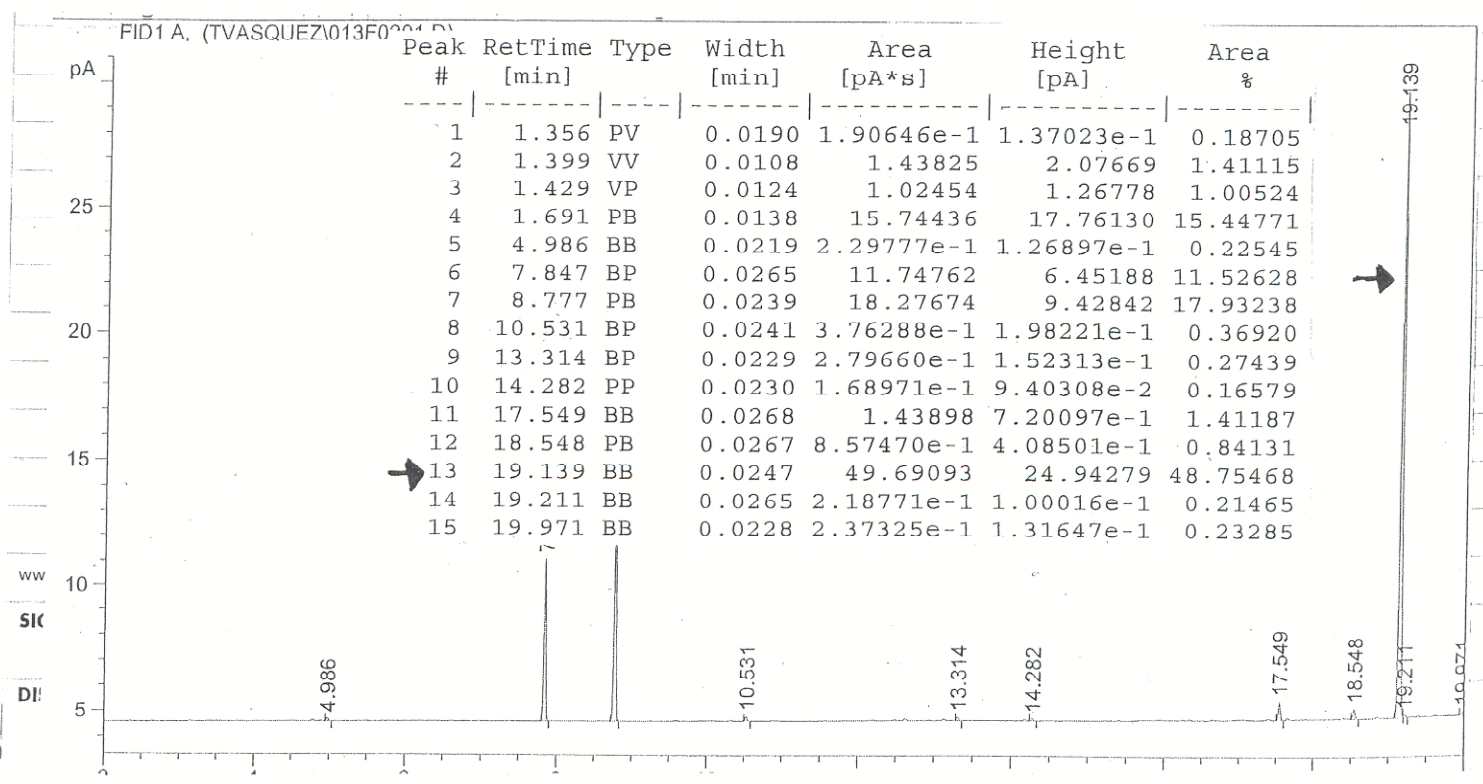




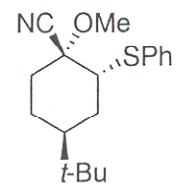

22

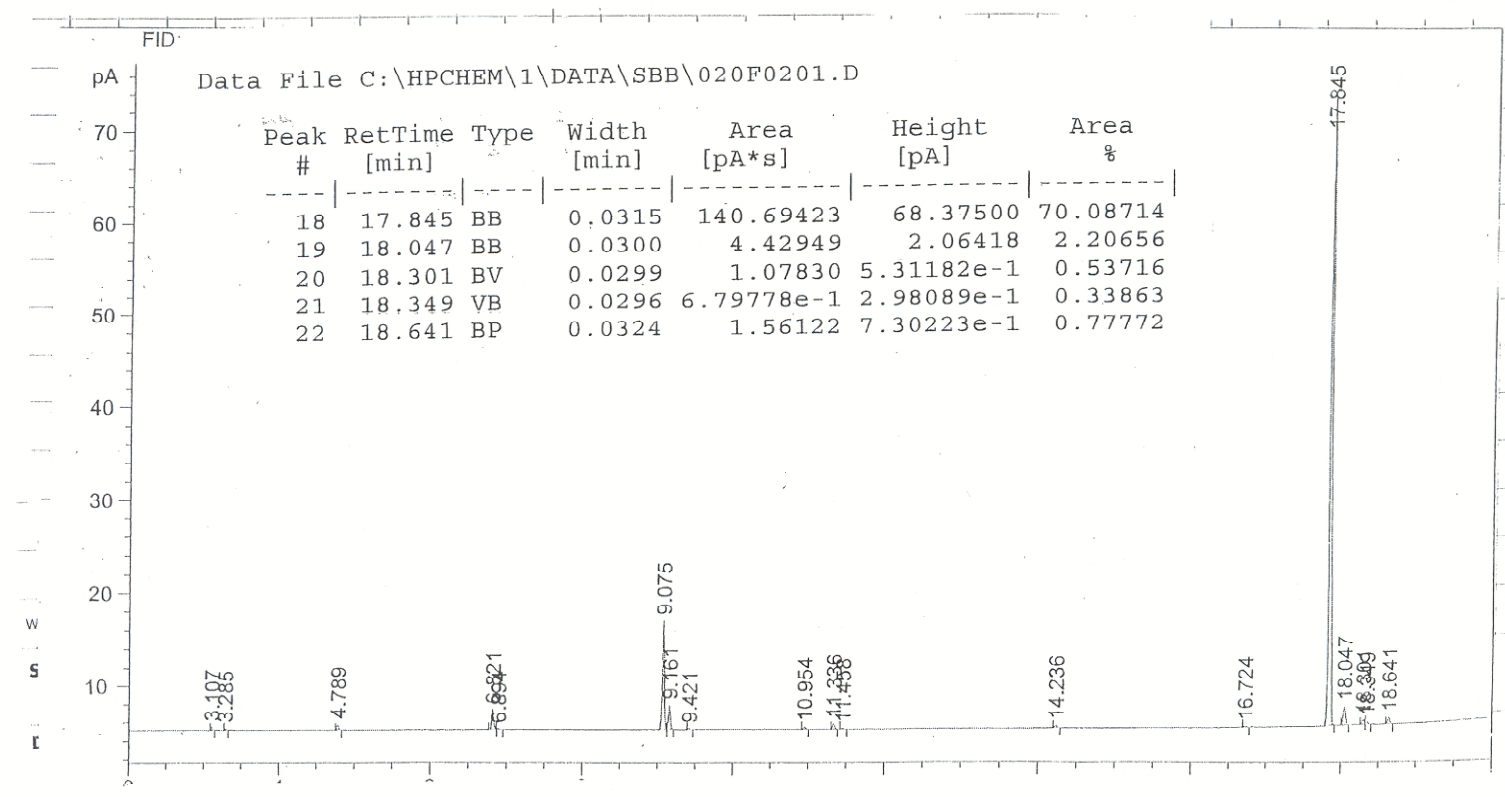




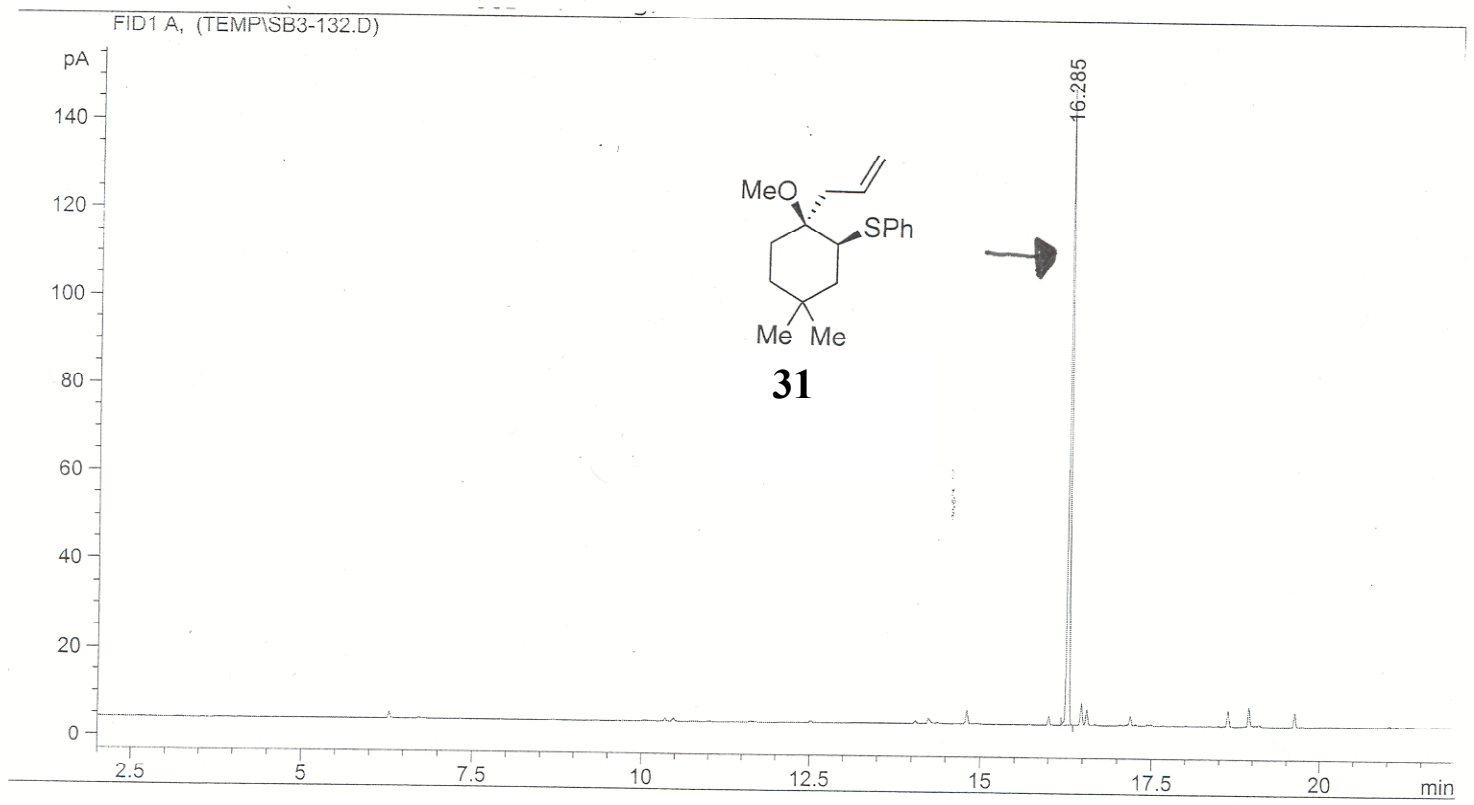

\begin{tabular}{|c|c|c|c|c|c|c|}
\hline $\begin{array}{c}\text { eak } \\
\# \\
--1\end{array}$ & $\begin{array}{l}\text { RetTime } \\
\text { [min] }\end{array}$ & Type & $\begin{array}{l}\text { Width } \\
\text { [min] }\end{array}$ & $\begin{array}{r}\text { Area } \\
{[\mathrm{pA} * \mathrm{~s}]}\end{array}$ & $\begin{array}{l}\text { Height } \\
{[p A]}\end{array}$ & $\begin{array}{c}\text { Area } \\
\circ\end{array}$ \\
\hline 16 & 1.112 & VV & $5.84 e-3$ & 4.20 & 13 & \\
\hline 17 & 1.120 & VV & $9.69 e-3$ & 1.00382 & & \\
\hline 18 & 1.158 & VB & $9.59 e-3$ & $4.71540 e-1$ & $7.75805 e-1$ & 0.0 \\
\hline 19 & 1.219 & $\mathrm{BB}$ & $9.99 e-3$ & 1.29535 & 2. 01857 & 68 \\
\hline 20 & 1.339 & $\mathrm{~PB}$ & 0.0113 & 50 & 2.37989 & 69 \\
\hline 21 & 1.420 & $\mathrm{BB}$ & 0.0118 & $4.43801 e-1$ & $5.73715 e-1$ & 92 \\
\hline 22 & 1.679 & $\mathrm{~PB}$ & 0.0143 & 9.67580 & 10.46872 & 01 \\
\hline 23 & 16.285 & $\mathrm{BB}$ & 0.0 & 297.52737 & 144.15672 & 0.61527 \\
\hline 24 & 22.138 & $\mathrm{BB}$ & 0.0306 & $5.10847 e-1$ & $1.99632 e-1$ & 0.00106 \\
\hline 25 & 23.827 & $\mathrm{BB}$ & 0.0423 & $5.99823 e-1$ & $1.68617 e-1$ & 0.00124 \\
\hline
\end{tabular}




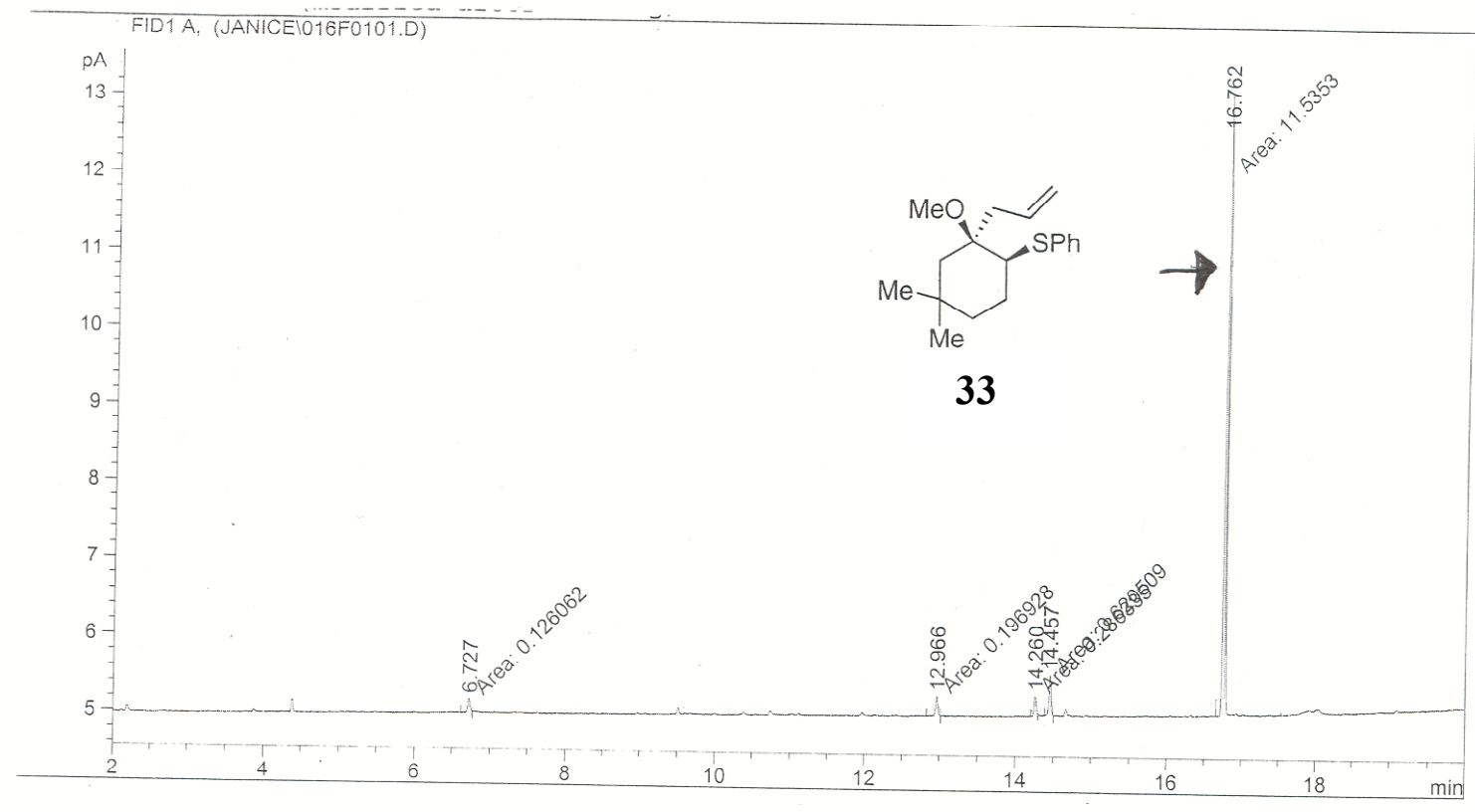

\begin{tabular}{|c|c|c|c|c|c|c|}
\hline $\begin{array}{c}\text { eak } \\
\#\end{array}$ & $\begin{array}{c}\text { RetTime } \\
\text { [min] }\end{array}$ & Type & $\begin{array}{c}\text { Width } \\
\text { [min] }\end{array}$ & $\begin{array}{r}\text { Area } \\
{\left[\mathrm{pA}^{*} \mathrm{~s}\right]}\end{array}$ & $\begin{array}{l}\text { Height } \\
{[p A]}\end{array}$ & $\begin{array}{c}\text { Area } \\
\frac{\circ}{0}\end{array}$ \\
\hline & & & & & & \\
\hline 16 & 1.042 & VV $\mathrm{X}$ & $7.74 e-3$ & 5. 01829 & 10.19171 & 0.00757 \\
\hline 17 & 1.088 & VV $\mathrm{X}$ & 0.0124 & $1.53558 e-1$ & $1.76180 e-1$ & 0.00023 \\
\hline 18 & 1.122 & VTV $\mathrm{X}$ & $9.20 e-3$ & 1.69855 & 2.75275 & 0.00256 \\
\hline 19 & 1.220 & VV $\mathrm{X}$ & 0.0100 & 4.42400 & 6.83680 & 667 \\
\hline 20 & 1.425 & $\mathrm{VB} \mathrm{T}$ & 0.0126 & $7.27560 \mathrm{e}-1$ & $8.62111 e-1$ & 0.00110 \\
\hline 21 & 1.684 & PB & 0.0138 & 12.74065 & 14.38497 & 0.01921 \\
\hline 22 & 6.727 & MM & 0.0202 & $1.26062 \mathrm{e}-1$ & 1. $04246 e-1$ & 0.00019 \\
\hline 23 & 12.966 & MM & 0.0 & $1.96928 \mathrm{e}-1$ & $1.51741 e-1$ & 0.00030 \\
\hline 24 & 14.260 & MM & 0.0247 & $2.86335 e-1$ & $1.93179 e-1$ & 0.00043 \\
\hline 25 & 14.457 & MM & 0.0255 & $6.20509 e-1$ & $4.05138 e-1$ & 0.00094 \\
\hline 26 & 16.762 & MM & 0.0245 & 11.53528 & 7.83000 & 0.01739 \\
\hline
\end{tabular}




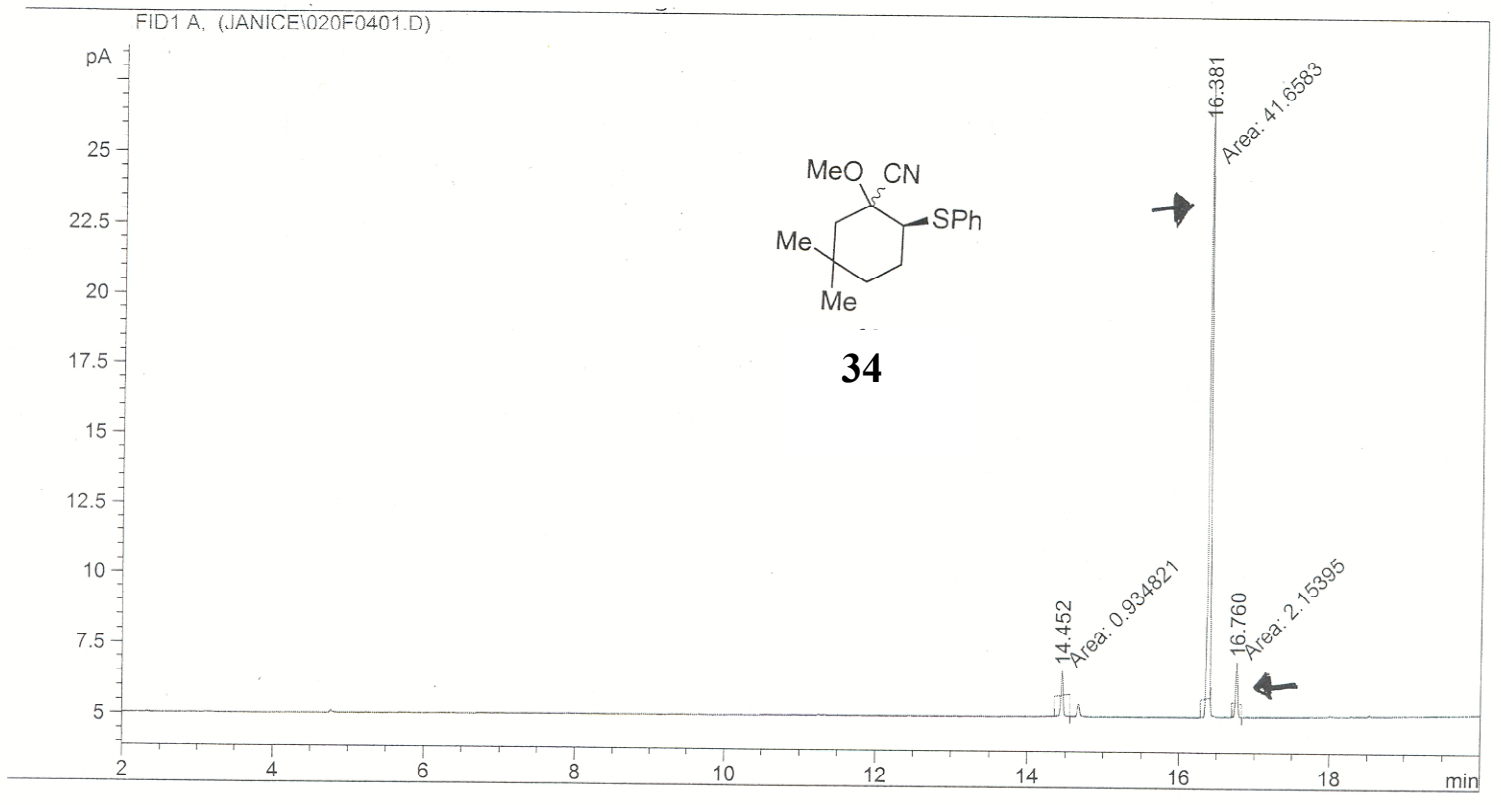

\begin{tabular}{|c|c|c|c|c|c|c|}
\hline $\begin{array}{c}\text { eak } \\
\#\end{array}$ & $\begin{array}{c}\text { RetTime } \\
\text { [min] }\end{array}$ & Type & $\begin{array}{c}\text { Width } \\
\text { [min] }\end{array}$ & $\begin{array}{r}\text { Area } \\
{\left[\mathrm{pA}^{*} \mathrm{~s}\right]}\end{array}$ & $\begin{array}{l}\text { Height } \\
\text { [pA] }\end{array}$ & $\begin{array}{c}\text { Area } \\
\frac{0}{0}\end{array}$ \\
\hline & & & & & 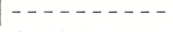 & ------ \\
\hline 1 & 0.685 & VV & $7.95 c-3$ & 3.8718401 & $6.73988 c \quad 1$ & 0.00265 \\
\hline 2 & 0.708 & VV & $4.49 e-3$ & 2.25101 & 7.53074 & 0.01541 \\
\hline 3 & 0.725 & VV & $5.10 e-3$ & $8.61292 e-1$ & 2.61028 & 0.00590 \\
\hline 4 & 0.755 & VV $\mathrm{S}$ & $4.60 e-3$ & 704.33765 & 2451.23340 & 4.82171 \\
\hline 5 & 0.808 & $\mathrm{VV} \mathrm{X}$ & $6.32 e-3$ & $1.57053 e-1$ & $3.61523 e-1$ & 0.00108 \\
\hline 6 & 0.858 & VB $S$ & $7.23 c 3$ & 1.3849504 & $2.94998 \subset 4$ & 24.80977 \\
\hline 7 & 1.042 & $\mathrm{VV} \mathrm{X}$ & $7.64 e-3$ & $3.94545 e-1$ & $8.16116 e-1$ & 0.00270 \\
\hline 8 & 1.124 & VB $X$ & 0.0117 & $5.50703 e-1$ & $6.62226 e-1$ & 0.00377 \\
\hline 9 & 1.220 & $\mathrm{BB}$ & $9.80 e-3$ & 1.19170 & 1.84169 & 0.00816 \\
\hline 10 & 1.430 & $\mathrm{~PB}$ & 0.0118 & $1.47567 e-1$ & $1.85785 e-1$ & 0.00101 \\
\hline 11 & 1.686 & $\mathrm{~PB}$ & 0.0133 & 3.14317 & 3.57214 & 0.02152 \\
\hline 12 & 14.452 & MM & 0.0187 & $9.34821 e-1$ & $8.32476 e-1$ & 0.00640 \\
\hline & 16.381 & MM & 0.0318 & 41.65826 & 21.79955 & 0.28518 \\
\hline & 16.760 & MM & 0.0253 & 2.15395 & 1.41833 & 0.01475 \\
\hline
\end{tabular}




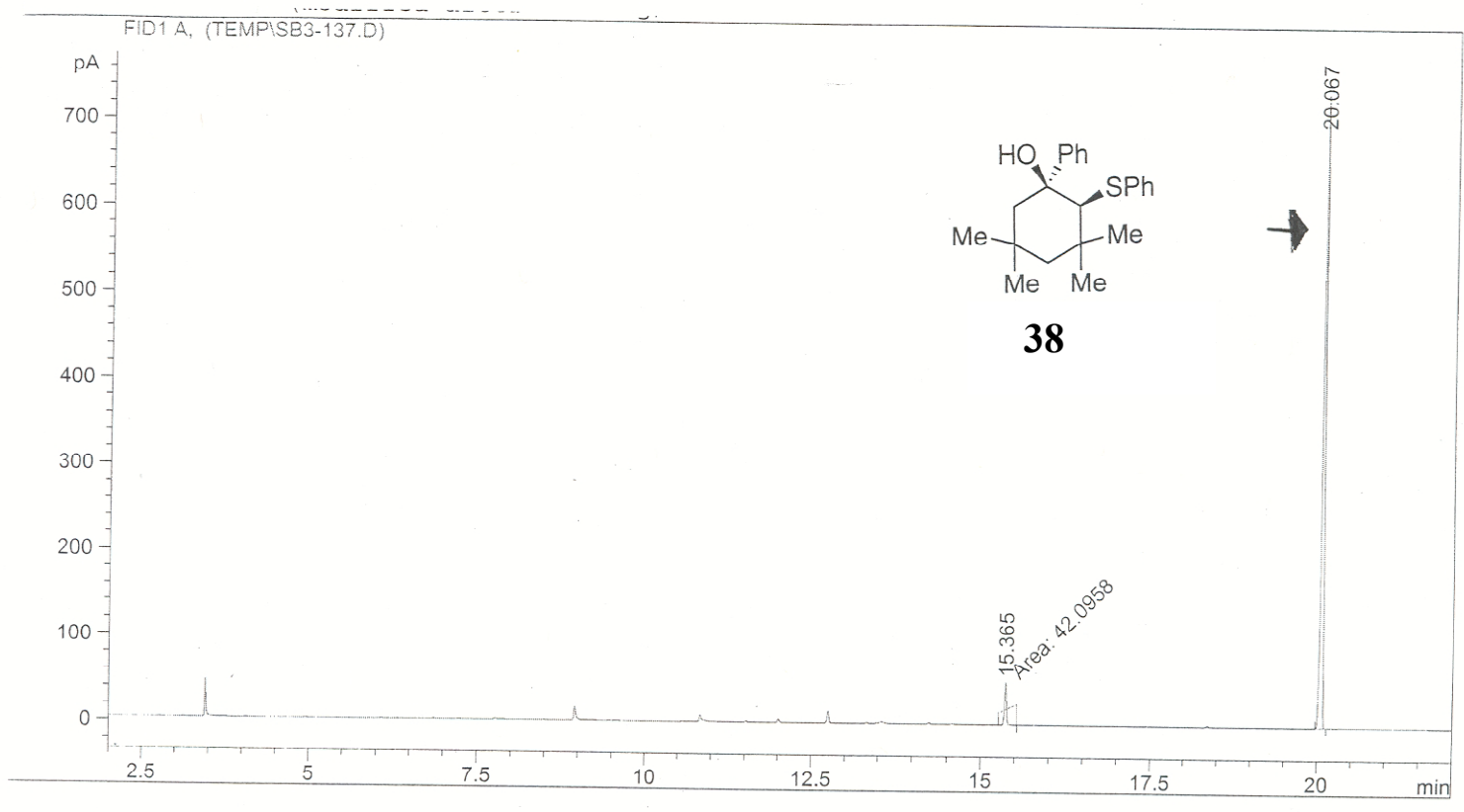

\begin{tabular}{|c|c|c|c|c|c|c|}
\hline $\begin{array}{c}\text { Peak } \\
\#\end{array}$ & $\begin{array}{c}\text { RetTime } \\
\text { [min] }\end{array}$ & Type & $\begin{array}{c}\text { Width } \\
\text { [min] }\end{array}$ & $\begin{array}{r}\text { Area } \\
{\left[p A^{*} s\right]}\end{array}$ & $\begin{array}{l}\text { Height } \\
\text { [pA] }\end{array}$ & $\begin{array}{c}\text { Area } \\
\frac{\circ}{0}\end{array}$ \\
\hline 16 & & & & - n- & & $\mid-\ldots$ \\
\hline $\begin{array}{l}16 \\
17\end{array}$ & $\begin{array}{l}15.365 \\
20.067\end{array}$ & $\begin{array}{l}\mathrm{M} I \mathrm{MI} \\
\mathrm{BR}\end{array}$ & 0.0226 & 42.09576 & 31.04755 & 0.09387 \\
\hline 18 & 24.864 & $\begin{array}{l}\mathrm{BB} \\
\mathrm{BB}\end{array}$ & $\begin{array}{l}0.0339 \\
0.0532\end{array}$ & $\begin{array}{l}1791.21411 \\
789861 e-1\end{array}$ & 734.81378 & 3.99441 \\
\hline & & & & $7.89861 e-1$ & $1.81021 \mathrm{e}-1$ & 0.00176 \\
\hline
\end{tabular}




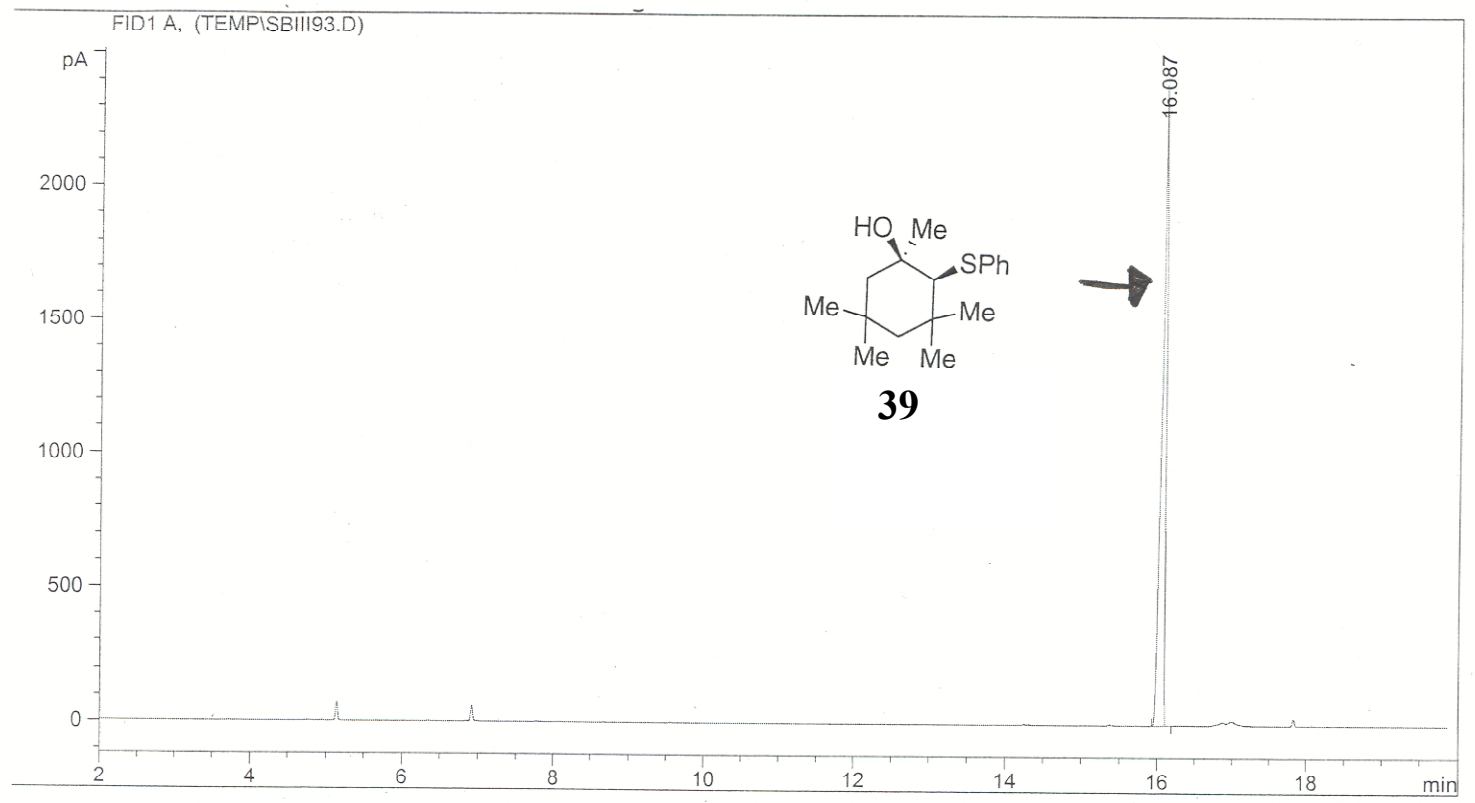

\begin{tabular}{|c|c|c|c|c|c|c|}
\hline $\begin{array}{c}\text { Peak } \\
\quad \#\end{array}$ & $\begin{array}{c}\text { RetTime } \\
\text { [min] }\end{array}$ & Type & $\begin{array}{l}\text { Width } \\
\text { [min] }\end{array}$ & $\begin{array}{r}\text { Area } \\
{[\mathrm{pA} * \mathrm{~s}]}\end{array}$ & $\begin{array}{l}\text { Height } \\
{[\mathrm{pA}]}\end{array}$ & $\begin{array}{c}\text { Area } \\
\frac{\circ}{6}\end{array}$ \\
\hline & & & & $1007016-1$ & 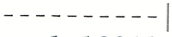 & 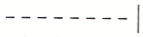 \\
\hline 15 & 1.346 & VV & 0.0125 & $9.91816 e-1$ & 1.19041 & 0.00027 \\
\hline 16 & 1.383 & $\mathrm{VV}$ & 0.0117 & 4.55637 & 5.76586 & 0.00126 \\
\hline 17 & 1.422 & $\mathrm{VP}$ & 0.0117 & 3.96906 & 5.17407 & 0.00110 \\
\hline 18 & 1.598 & $\mathrm{VP}$ & 0.0146 & $3.47331 e-1$ & $3.73134 \mathrm{e}-1$ & $9.617 e-5$ \\
\hline 19 & 1.682 & BB & 0.0133 & 80.94323 & 94.37732 & 0.02241 \\
\hline 20 & 1.773 & BB & 0.0166 & $2.49445 e-1$ & $1.89603 e-1$ & $6.907 e-5$ \\
\hline 21 & 16.087 & BB & 0.0479 & 2150.56348 & 2389.74634 & 2.53375 \\
\hline 22 & 20.998 & $\mathrm{BB}$ & 0.0503 & 1.11736 & 2. $67129 e-1$ & 0.00031 \\
\hline 23 & 21.530 & $\mathrm{BB}$ & 0.0469 & 2.15408 & $5.57070 e-1$ & 0.00060 \\
\hline
\end{tabular}




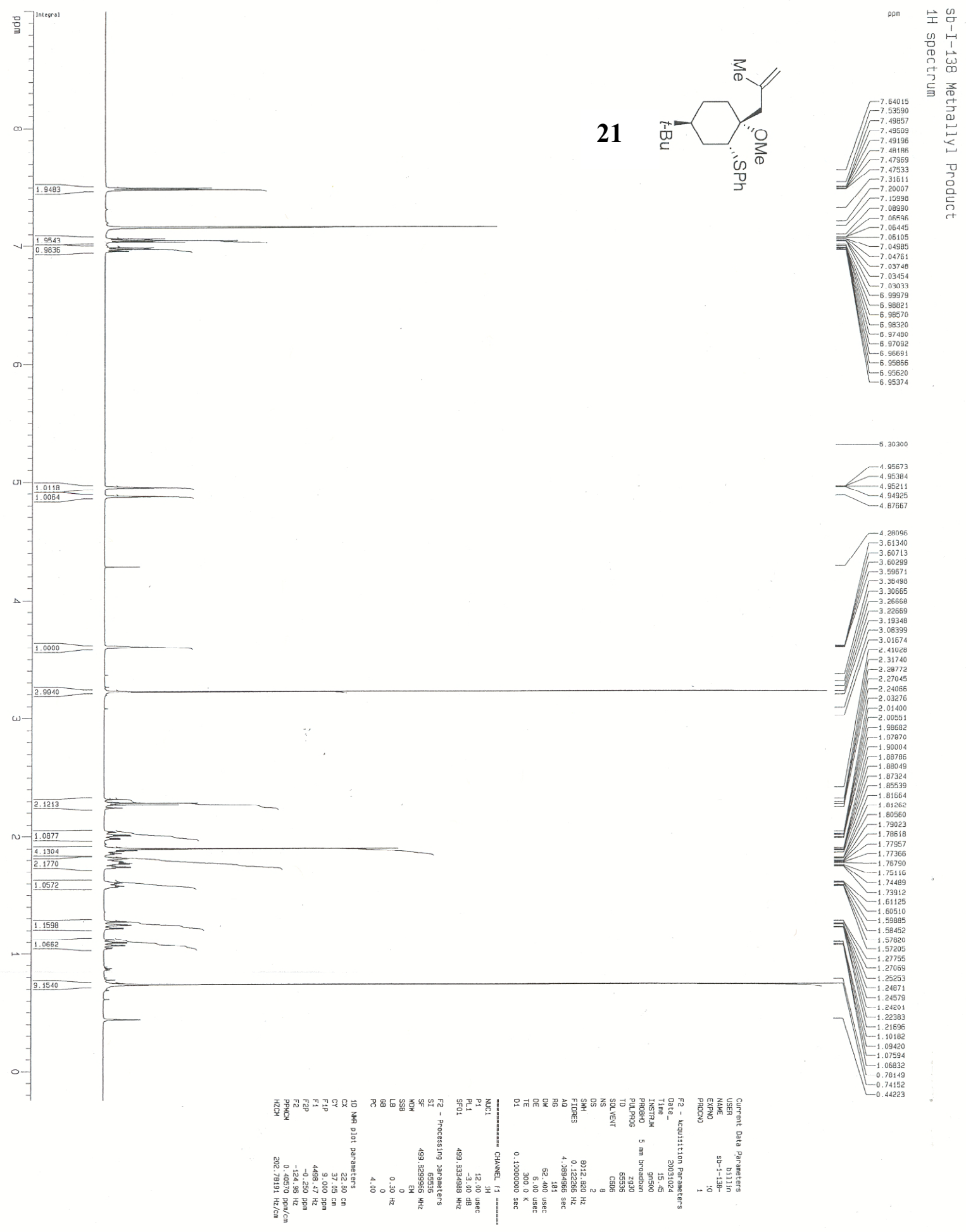




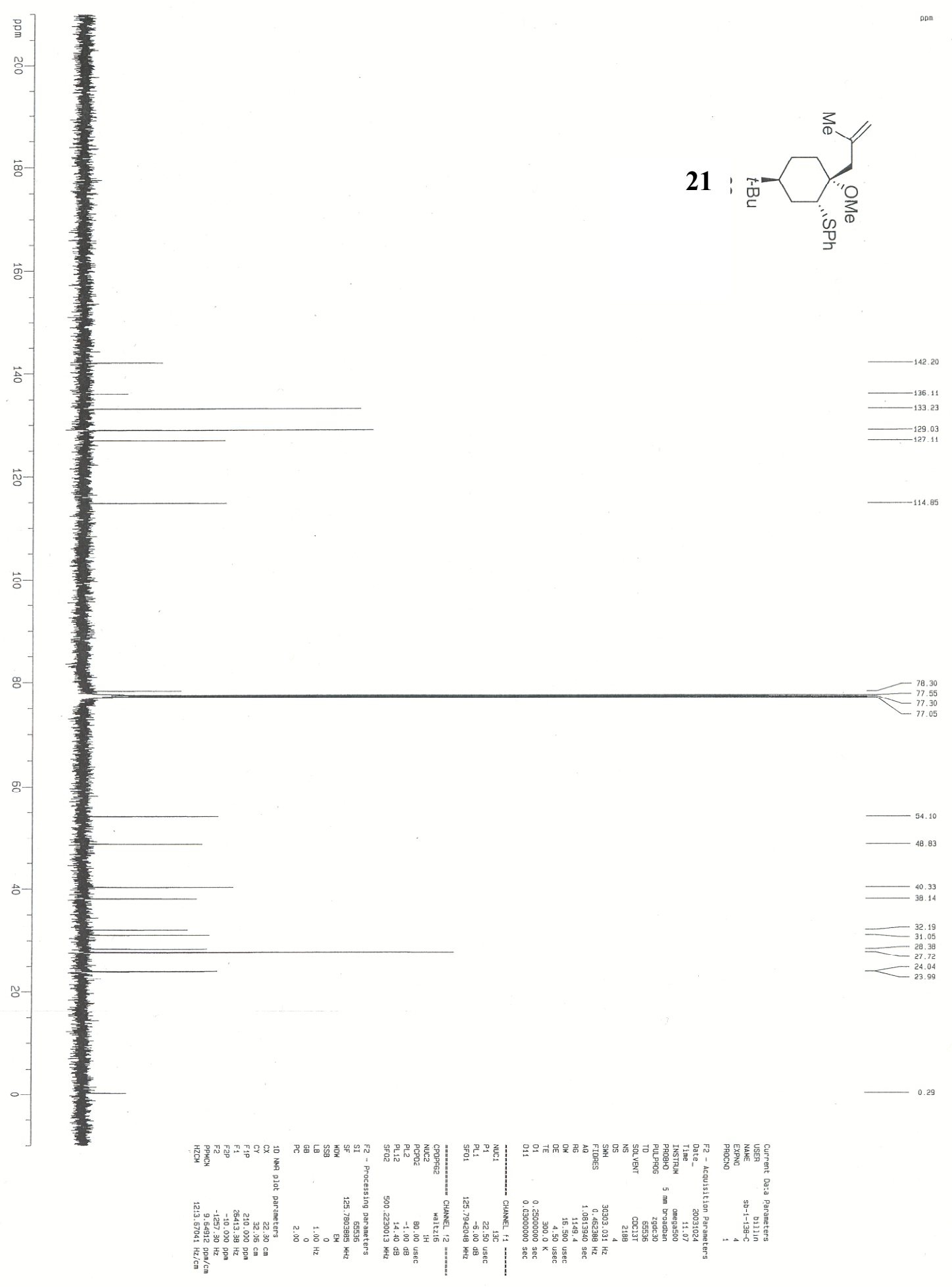




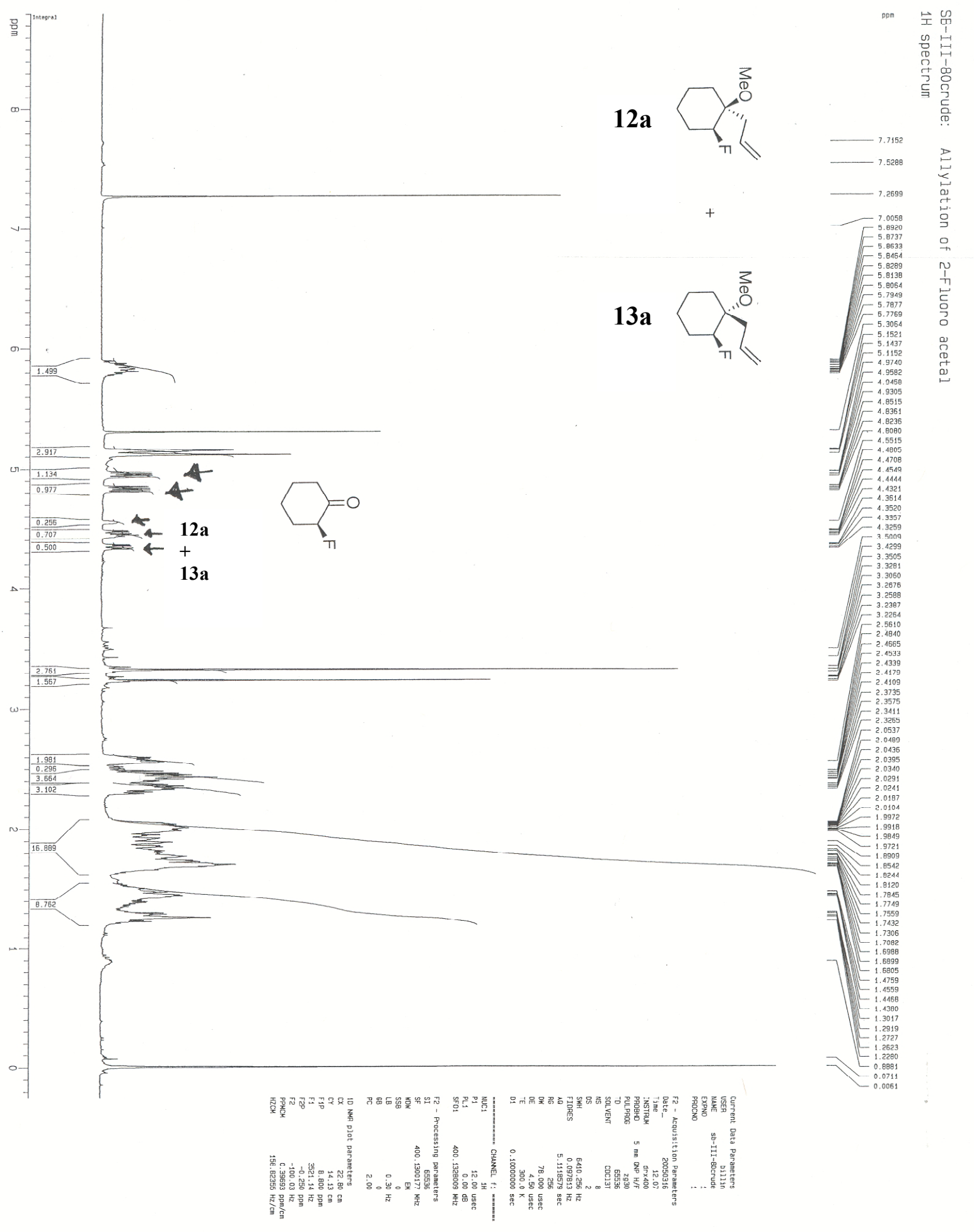

